\title{
Analysis of Morphology Based Horticultural Features through Clustering Methods
}

\author{
K.Deb \\ Department of Computer \\ Science \& Engineering \\ Jadavpur University \\ Kolkata,India
}

\author{
A.Hazra \\ Department of Computer \\ Science \& Engineering \\ Jadavpur University \\ Kolkata,India
}

\author{
S.Kundu \\ Department of Computer \\ Science \& Engineering \\ Jadavpur University \\ Kolkata,India
}

\author{
P.Hazra \\ Faculty of Horticulture, \\ Bidhan Chandra Krishi \\ Viswavidyalaya, \\ Kalyani,Nadia,India
}

\begin{abstract}
Cluster analysis is a prime Pattern Recognition method used to categorize sample patterns in a population by means of forming different clusters by assigning cluster memberships to the sample patterns depending on the feature similarity relationship among different patterns. Patterns displaying dissimilar feature values are assigned different cluster memberships whereas patterns carrying similar feature values are placed into same cluster. Searching the relationship among horticultural data has become a major research area in Pattern Recognition. In this paper we have used the morphological features for describing the characteristics of Tomato leaves and fruits belonging to different classes. Morphological feature values are extracted from different tomato leaf and fruiting habit samples to analyze through K-Means and Two-step clustering techniques to segment leaf and fruit samples into separate clusters according to their species owing to categorize them. Our experimentation also compares and discusses about the importance of the features which are obtained through K-Means and Two-step Clustering technique, may be useful for leaf and fruit species categorization.
\end{abstract}

Keywords: Cluster analysis, K-Means Clustering, Two-step clustering, Pattern Recognition, Horticulture, Morphological Feature.

\section{INTRODUCTION}

Economic growth of a nation depends highly on its agricultural and horticultural development. As different cultivars may need specifically different cultivation processes for better growth and quality development hence it is very much needed to identify the horticultural cultivars belonging to different classes independently, so that appropriate cultivation means can be applied for specific cultivar. Thus horticultural species categorization is a very important task for cultivation. This task of categorization is easy to perform for the crops with small number of species variations, but the task becomes a tough one if huge numbers of crop species are to be dealt with. Horticultural vegetable like tomato have large variety of species found all around the globe. The high variation of morphological feature values of tomato leaf and fruit among different tomato species is a prominent indicator of species diversity upon which cluster analysis would be applied to form different tomato-species clusters and thus categorizing the tomato species depending on their cluster memberships. This automatic method of species categorization through clustering exhibits high level of accuracy and requires trifle time compare to manual process. Cluster analysis also produces some distinguishing results through which the feature importance of leaves and fruits could be predicted which may be of very useful while classifying the particular plant species.

\section{BASIC CLUSTER ANALYSIS}

Cluster analysis is an important analytical procedure used for the purpose of analysing data. Cluster analysis is widely used in different research areas like machine learning, pattern recognition, market research, digital image processing, Biology etc. Basically, clustering divides the sample entities into different groups called clusters depending on similarity present between entities. Entities placed into same cluster, bear great deal of similar features where as entities belonging to different clusters don't have that much of feature similarity like same cluster entities. All the member entities of a cluster can be represented by the cluster centre of that cluster. Now with the compact cluster formation, obtaining information from the original entity set can be sufficiently reduced to collecting information about a small number of clusters. Information obtained from the clusters can be very effective for purposes like entity classification, identification etc.

Clustering algorithms can be classified into categories such as: Partitional Clustering and Hierarchical Agglomerative Clustering. We discuss these two clustering techniques briefly in the following-

Partitional Clustering starts with some initial clusters. For each of the initial clusters, a cluster center is calculated by fulfilling the optimality condition. Sample objects are placed in different clusters depending on the smallest distance criterion i.e. a sample object is placed in that cluster whose cluster center is minimum distance away from the sample object. Sample input data are partitioned into the initial clusters. In the next step, cluster centers are recalculated and objects are again placed in different clusters depending on the new calculated cluster centers. This process of cluster center 
recalculation and placing the objects in clusters continues unless the placement of the objects in the clusters remains unaltered between two successive rotations.

Hierarchical Agglomerative Clustering algorithm starts with some single clusters depending on the size of the input data set. Number of initial single clusters is equal to the size of the input data set and each of the input patterns belongs to different cluster. Now as the algorithm moves, at each of the successive steps, merging of the cluster pairs having highest level of attribute similarities is performed.

\section{APPLIED CLUSTERING METHODS}

\subsection{K-means Cluster Analysis}

$\mathrm{K}-\mathrm{Means}$ cluster analysis falls into the category of partional clustering algorithm. K-Means cluster analysis is used for analysing the feature data set.

Let's assume that $\mathrm{P}$ number of sample patterns is to be clustered. A pattern set $D=\left\{d_{1}, d_{2}, . ., d_{P}\right\}$ represents the sample patterns .The characteristics of each sample pattern is represented by $\mathrm{Z}$ number of features, which constitute the feature set $F=\left\{f_{1}, f_{2}, \ldots \ldots, f_{Z}\right\}$. Now for each of the $Z$ features, $P$ different feature values are obtained from each of the $P$ sample patterns. The feature values associated to a feature $f_{X}$ forms the individual feature value set $\mathrm{IFV}_{\mathrm{X}}=\left\{\right.$ ifv $_{1 X}$, if $\left._{2 \mathrm{X}}, \ldots, \mathrm{ifv}_{\mathrm{NX}}, \ldots, \mathrm{ifv}_{\mathrm{PX}}\right\}$, of size $\mathrm{P}$, where ' ifv $_{\mathrm{NX}}$ ', an element of the set $\mathrm{IFV}_{\mathrm{X}}$, denotes the feature value of $\mathrm{N}$ th sample pattern with respect to feature $f_{X}$. K-Means cluster analysis is done on each IFV, to place $\mathrm{P}$ patterns in $\mathrm{k}$ (user given value) different clusters depending on the values of the elements of the IFV. K-Means clustering initially selects $\mathrm{k}$ patterns out of $\mathrm{P}$ patterns as initial clusters. Each cluster is represented by a cluster center. The value of each initial cluster center will be one of the elements of the IFV chosen randomly with uniqueness condition that same IFV element can't be placed into more than one initial clusters. Also the cluster membership of a particular IFV element remains same till the end of the clustering process.

Let's consider that K-Means clustering is applied to $\mathrm{IFV}_{\mathrm{X}}$. This will lead to the formation of $\mathrm{k}$ clusters each having a cluster center. Let's consider that the cluster center of ' $\mathrm{i}$ ' th cluster is denoted by $\mathrm{CC}_{\mathrm{i}}$. Let's denote the value of cluster center $C_{i}$ by $V_{C C}$. Now ' $M$ ' th data pattern $d_{M}$ will be placed into the ' $\mathrm{i}$ ' th cluster by satisfying the condition $\operatorname{Dis}\left(d_{M}, C_{i}\right)<\operatorname{Dis}\left(d_{M}, C C_{j}\right)$, for all $j \neq i$, where $\operatorname{Dis}\left(d_{M}, C_{i}\right)$ is the distance between the data pattern $\mathrm{d}_{\mathrm{M}}$ and the ' $\mathrm{i}$ ' th cluster center $\mathrm{CC}_{\mathrm{i}}$ and $\operatorname{Dis}\left(\mathrm{d}_{\mathrm{M}}, \mathrm{CC}_{\mathrm{j}}\right)$ is the distance between $\mathrm{d}_{\mathrm{M}}$ and another ' $\mathrm{j}$ ' th cluster center $\mathrm{CC}_{\mathrm{j}}$. Now, $\operatorname{Dis}\left(\mathrm{d}_{\mathrm{M}}, \mathrm{CC}_{\mathrm{i}}\right)$ can be calculated as per the following equation-

$\operatorname{Dis}\left(\mathrm{d}_{\mathrm{M}}, \mathrm{CC}_{\mathrm{i}}\right)=\mid$ ifv $_{\mathrm{MX}}-\mathrm{VCC}_{\mathrm{i}} \mid$

The values of $\mathrm{k}$ cluster centers will be recalculated again and again unless no new member is placed in clusters. The updated value of a cluster center is the calculated average of the values member elements of the cluster. So $\mathrm{VCC}_{\mathrm{i}}$ is updated as -

$\mathrm{VCC}_{\mathrm{i}}=\left(\mathrm{vme}_{1 \mathrm{i}}+\mathrm{vme}_{2 \mathrm{i}}+\ldots . .+\mathrm{vme}_{\mathrm{li}}+\ldots .+\mathrm{vme}_{\mathrm{si}}\right) \cdot(1 / \mathrm{s})$

, where ' $\mathrm{vme}_{1 \mathrm{l}}$ ' denotes the value of ' $\mathrm{l}$ ' th member element of ' $i$ ' th cluster having ' $s$ ' number of member elements of the ' $i$ ' th cluster. Basically value of each member element is an element of set $\mathrm{IFV}_{\mathrm{X}}$.

In the following, we summarize different steps of K-Means algorithm done on the set $\mathrm{IFV}_{\mathrm{X}}$ -
1) Randomly choose k number of initial cluster centers out of $P$ elements of the IFV $_{X}$ set.

2) Place each pattern from pattern set $D$ in the cluster whose cluster center is closest to it by calculating the pattern-cluster distance as per equation (1)

3 ) Recompute cluster centers as per equation (2), depending on the recent placement of the elements into the cluster and reassign the elements to its closest cluster based on the newly computed centers.

4) Repeat step 2 and 3 until there is no alteration in the cluster memberships.

\subsection{Two step Cluster Analysis}

Two-step cluster analysis belongs to the class of Hierarchical Agglomerative Clustering. Consider the pattern set $\mathrm{D}$ of size $\mathrm{P}$ and feature set $\mathrm{F}$ of size $\mathrm{Z}$ as mentioned in section 3.1. Values of $\mathrm{Z}$ features are extracted from each pattern. Hence values related to $\mathrm{Z}$ features, extracted from $\mathrm{I}$ th pattern $\mathrm{d}_{\mathrm{I}}$, forms the values of features set $\mathrm{VF}_{\mathrm{I}}=\left\{\mathrm{vf}_{\mathrm{I} 1}, \mathrm{vf}_{\mathrm{I} 2}, \ldots ., \mathrm{vf}_{\mathrm{IJ}}, \ldots \mathrm{vf}_{\mathrm{IZ}}\right\}$, where $\mathrm{vf}_{\mathrm{IJ}}$ is the value of $\mathbf{J}$ th feature extracted from $\mathrm{I}$ th pattern. Each pattern is represented by its VF set. Now the values of all features extracted from all $\mathrm{P}$ patterns build a set of all values of features $\mathrm{AVF}=\left\{\mathrm{VF}_{1}, \mathrm{VF}_{2}, \ldots \mathrm{VF}_{\mathrm{I}} \ldots, \mathrm{VF}_{\mathrm{P}}\right\}$. Two-step cluster analysis is performed on the set AVF .Two-step clustering initially forms some sub-clusters and places $\mathrm{P}$ patterns into them. Each pattern is described by its VF values, so each subcluster contains the VF value of the member pattern. Let's consider that $\mathrm{sc}_{\mathrm{A}}$ and $\mathrm{sc}_{\mathrm{B}}$ are two sub-clusters containing pattern $d_{I}$ and $d_{K}$ respectively. Now the distance between $\mathrm{sc}_{\mathrm{A}}$ and $\mathrm{sc}_{\mathrm{B}}$ is calculated by calculating the Euclidean Distance between $\mathrm{VF}_{\mathrm{I}}$ and $\mathrm{VF}_{\mathrm{K}}$, denoted by $\mathrm{ED}\left(\mathrm{VF}_{\mathrm{I}}, \mathrm{VF}_{\mathrm{K}}\right)$, in the following equation-

$\mathrm{ED}\left(\mathrm{VF}_{\mathrm{I}}, \mathrm{VF}_{\mathrm{K}}\right)=\left(\left(\mathrm{vf}_{\mathrm{I} 1}-\mathrm{vf}_{\mathrm{K} 1}\right)^{2}+\left(\mathrm{vf}_{\mathrm{I} 2}-\mathrm{vf}_{\mathrm{K} 2}\right)^{2}+. .+\left(\mathrm{vf}_{\mathrm{IZ}}-\mathrm{vf}_{\mathrm{KZ}}\right)^{2}\right)^{1 / 2}$

The Two-step algorithm operates on AVF set in the following manner-

1) Place all the $P$ sample patterns into different sub clusters depending on the values of the features set (VF) of each pattern. So each sub-cluster contain the VF set of a pattern.

2) Calculate the distance between sub clusters using equation (3). Merge two nearest sub-clusters(clusters with minimum distance between each other) into one cluster to form new clusters.

Repeat the process of nearest cluster merging between the new clusters until desired number of clusters are formed.

\section{EXPERIMENTAL RESULTS}

In this paper we have used the best selected morphological features ${ }^{[1]}$ to perform K-Means and Two-step clustering with the help of IBM SPSS statistics 20 data mining tool and there by comparing the cluster building abilities of these features. This comparison will give better visibility about the impact of the features in machine vision solutions. The morphological features used in our experiment are listed below -

\section{Leaf Features}

1) Major Axis

2) Minor Axis

3) Aspect Ratio

4) Eccentricity

5) Area

6) Rectangularity 
7) Diameter

8) Compactness

9) Perimeter Ratio of Major Axis-Minor Axis

10) Perimeter Ratio of Diameter

11) Concavity

12) R-Factor

\section{Fruit Features}

1) Branch Length

2) Branch Width

3) Length Width Ratio

4) Area

5) Perimeter

6) Equivalent Diameter

7) Rectangularity

8) Diameter

9) Perimeter Ratio of Branch Length-Branch Width

10) Perimeter Ratio of Diameter

11) Convexity

12) Solidity

13) On Pixels

14) Narrow-Factor

\subsection{K-Means Clustering Results}

K-Means clustering is performed individually on feature values, related to each individual leaf and fruit features, extracted from all sample leaf and fruit patterns.

K-Means process starts by randomly selecting 15 and 14 initial cluster centers of individual leaf features (Table 1) and individual fruit features (Table 2 ) respectively.

Table 1- Randomly chosen Initial cluster centers for individual leaf features

\begin{tabular}{|l|r|r|r|r|r|r|r|r|}
\hline \multicolumn{10}{|c|}{ Initial Cluster Centers } \\
\cline { 2 - 9 } & \multicolumn{1}{|c|}{1} & \multicolumn{1}{|c|}{ Cluster } \\
\hline $\begin{array}{l}\text { Major } \\
\text { Axis }\end{array}$ & 479 & 390 & 419 & 339 & 370 & 435 & 587 & 503 \\
\hline
\end{tabular}

\begin{tabular}{|r|r|r|r|r|r|r|r|}
\hline \multicolumn{10}{|c|}{ Initial Cluster Centers } \\
\cline { 2 - 8 } & \multicolumn{1}{|c|}{ Cluster } \\
\hline & 9 & 10 & 11 & 12 & 13 & 14 & 15 \\
\hline Major Axis & 599 & 533 & 519 & 575 & 621 & 654 & 557 \\
\hline
\end{tabular}

\begin{tabular}{|c|c|c|c|c|c|c|c|c|}
\hline \multicolumn{9}{|c|}{ Initial Cluster Centers } \\
\hline & \multicolumn{8}{|c|}{ Cluster } \\
\hline & 1 & 2 & 3 & 4 & 5 & 6 & 7 & 8 \\
\hline $\begin{array}{l}\text { Minor } \\
\text { Axis }\end{array}$ & 167 & 321 & 216 & 197 & 254 & 182 & 279 & 291 \\
\hline
\end{tabular}

\begin{tabular}{|c|c|c|c|c|c|c|c|}
\hline & \multicolumn{7}{|c|}{ Cluster } \\
\hline & 9 & 10 & 11 & 12 & 13 & 14 & 15 \\
\hline Minor Axis & 150 & 303 & 366 & 404 & 266 & 230 & 245 \\
\hline
\end{tabular}

\begin{tabular}{|l|c|c|c|c|c|c|c|}
\hline & \multicolumn{7}{|c|}{ Intial Cluster Centers } \\
\cline { 2 - 9 } & 1 & 2 & 3 & 4 & 5 & 6 & 7 \\
\hline $\begin{array}{l}\text { Aspect } \\
\text { Ratio }\end{array}$ & 2.523810 & 2.795276 & 3.086207 & 3.264000 & 3.175000 & 2.081340 & 3.462264 \\
\hline
\end{tabular}
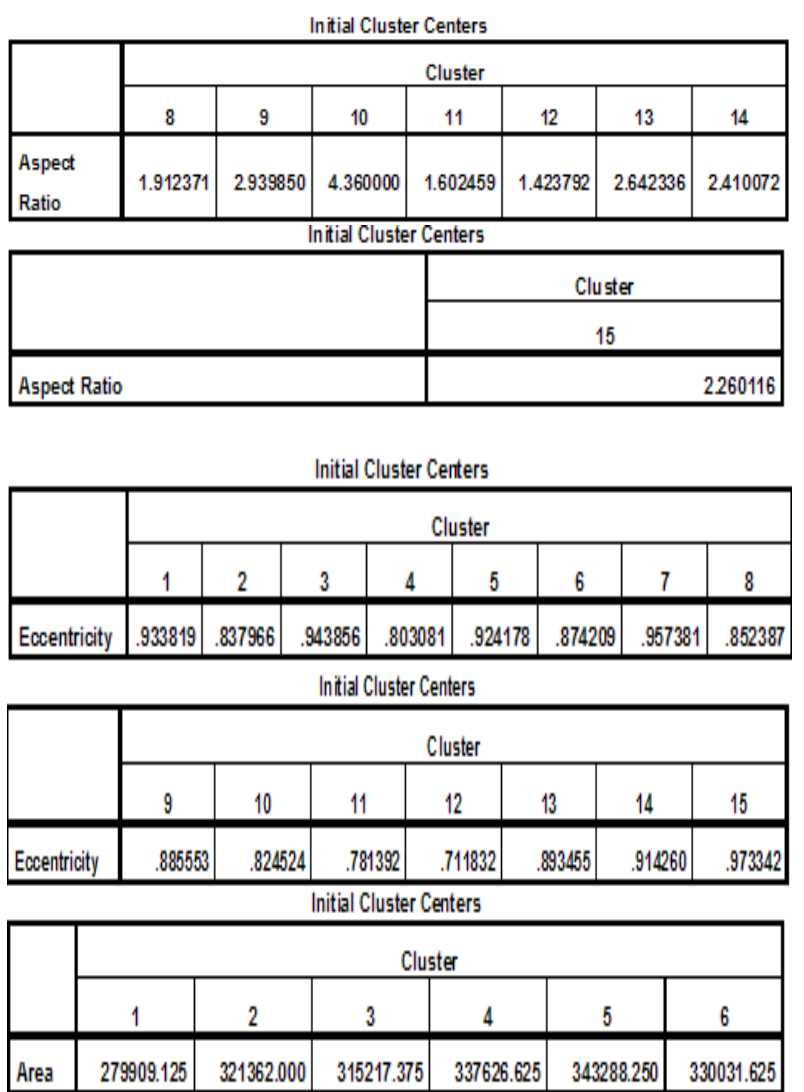

Initial Cluster Centers

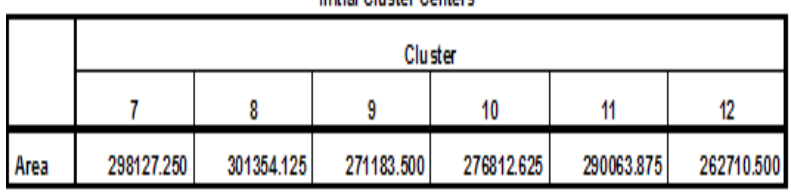
Intial Cluster Centers

\begin{tabular}{|l|r|r|r|}
\hline \multirow{2}{*}{} & \multicolumn{3}{|c|}{ Cluster } \\
\cline { 2 - 4 } & 13 & 14 & \multicolumn{1}{|c|}{15} \\
\hline Area & 285034.125 & 304995.625 & 295351.000 \\
\hline
\end{tabular}

Initial Cluster Centers

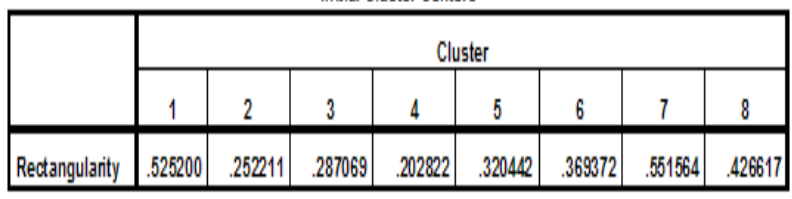

Intial Cluster Centers

\begin{tabular}{|c|c|c|c|c|c|c|c|}
\hline \multirow{2}{*}{} & \multicolumn{7}{|c|}{ Cluster } \\
\cline { 2 - 8 } & 9 & 10 & 11 & 12 & 13 & 14 & 15 \\
\hline Rectangulanity & .621876 & .5869934 & .792047 & .883636 & .504833 & .463316 & .403556 \\
\hline
\end{tabular}

\begin{tabular}{|r|r|r|r|r|r|r|r|r|}
\hline & \multicolumn{7}{|c|}{ Cluster } \\
\cline { 2 - 9 } & 1 & 2 & 3 & 4 & 5 & 6 & 7 & 8 \\
\hline Diameter & 414 & 316 & 398 & 441 & 356 & 492 & 470 & 614 \\
\hline
\end{tabular}



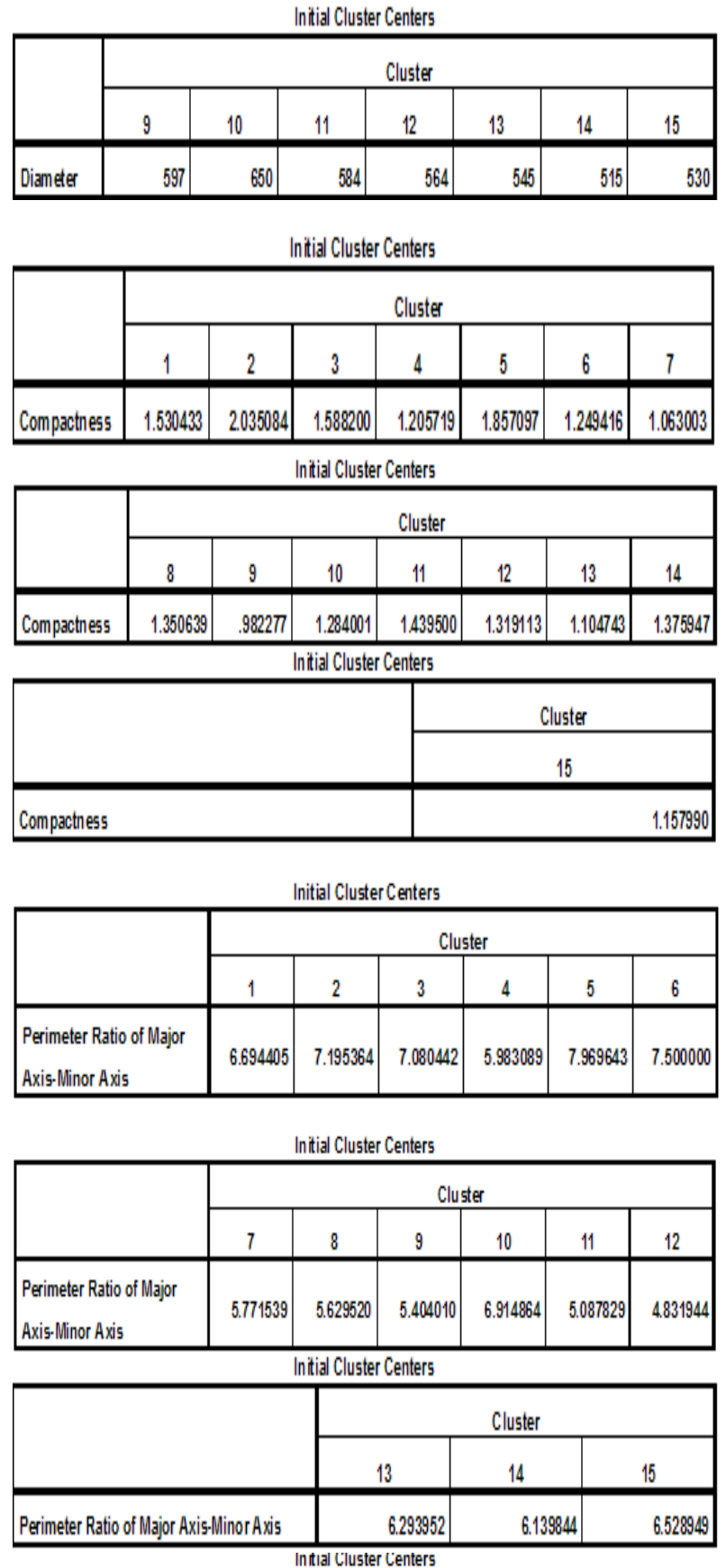

\begin{tabular}{|l|c|c|c|c|c|c|}
\hline \multirow{2}{*}{} & \multicolumn{7}{|c|}{ Intial Cluster Centers } \\
\cline { 2 - 7 } & 1 & 2 & 3 & 4 & 5 & 6 \\
\hline $\begin{array}{l}\text { Perimeter Ratio of } \\
\text { Diameter }\end{array}$ & 8.718986 & 13.753165 & 11.278894 & 13.200000 & 12.536517 & 8.997152 \\
\hline
\end{tabular}

\begin{tabular}{|c|c|c|c|c|c|c|}
\hline & \multicolumn{6}{|c|}{ Cluster } \\
\cline { 2 - 7 } & 7 & 8 & 9 & 10 & 11 & 12 \\
\hline Perimeter Ratio of Diameter & 7.576137 & 7.902203 & 8.466625 & 9.509748 & 8.289941 & 10.629945 \\
\hline
\end{tabular}
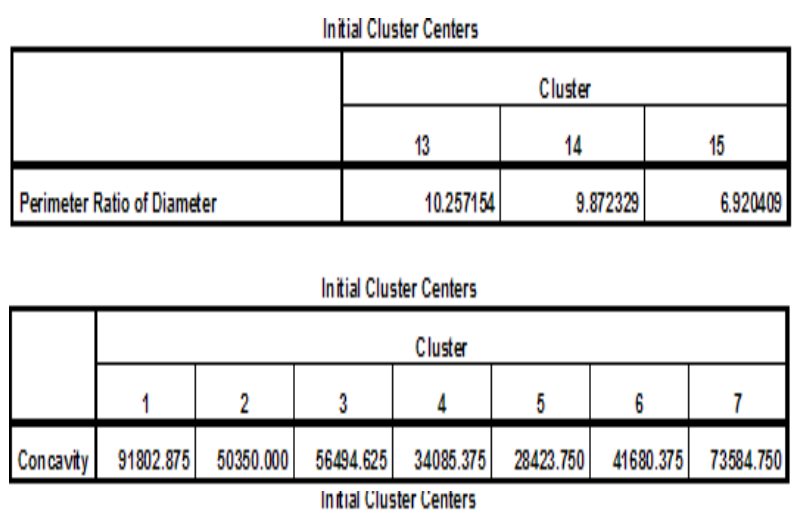

\begin{tabular}{|c|c|c|c|c|c|c|}
\hline \multirow{2}{*}{} & \multicolumn{7}{|c|}{ Cluster } \\
\cline { 2 - 7 } & 8 & 9 & 10 & 11 & 12 & 13 \\
\hline Concavity & 70357.875 & 100528.500 & 94899.375 & 81648.125 & 108918.500 & 86677.875 \\
\hline
\end{tabular}
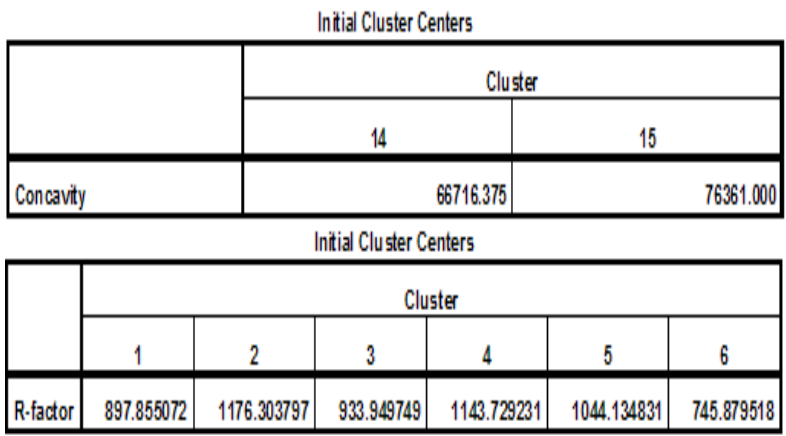
Initial Cluster Centers
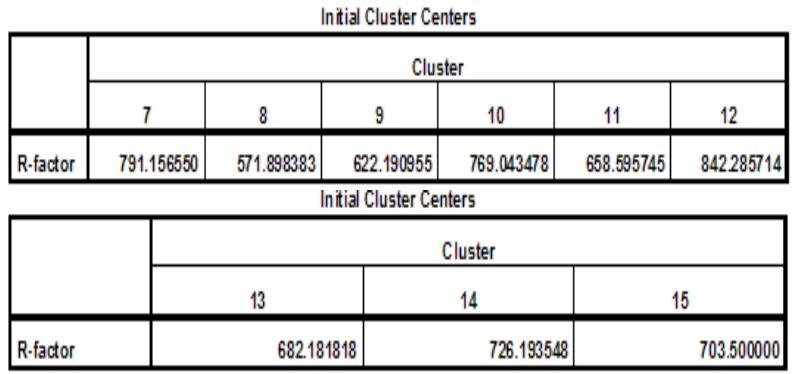

Table 2- Randomly chosen Initial cluster centers for individual fruit features

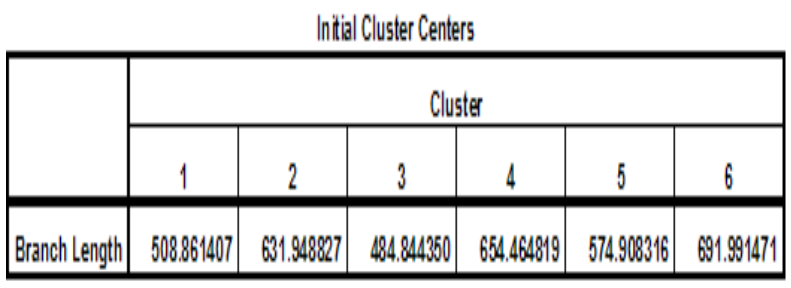

Initial Cluster Centers

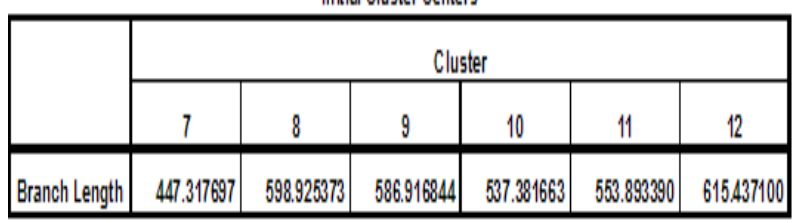


International Journal of Computer Applications Technology and Research

Volume 2- Issue 5, 557 - 586, 2013, ISSN: 2319-8656

Intial Cluster Centers

\begin{tabular}{|c|c|c|}
\hline \multirow{2}{*}{} & \multicolumn{2}{|c|}{ Cluster } \\
\cline { 2 - 3 } & 13 & 14 \\
\hline Branch Length & 520.869936 & 673.978678 \\
\hline
\end{tabular}

Initial Cluster Centers

\begin{tabular}{|c|c|c|c|c|c|c|}
\hline & \multicolumn{6}{|c|}{ Cluster } \\
\cline { 2 - 7 } & 1 & 2 & 3 & 4 & 5 & 6 \\
\hline Branch Width & 411.292111 & 358.754797 & 475.837953 & 321228145 & 382.771855 & 295.710021 \\
\hline
\end{tabular}

Initial Cluster Centers

\begin{tabular}{|c|c|c|c|c|c|c|}
\hline & \multicolumn{7}{|c|}{ Cluster } \\
\cline { 2 - 7 } & 7 & 8 & 9 & 10 & 11 & 12 \\
\hline Branch Width & 447.317697 & 496.852878 & 460.827292 & 256.682303 & 424.801706 & 340.742004 \\
\hline
\end{tabular}
Initial Cluster Centers

\begin{tabular}{|l|c|c|}
\hline \multirow{2}{*}{} & \multicolumn{2}{|c|}{ Cluster } \\
\cline { 2 - 3 } & 13 & 14 \\
\hline Branch Width & $235.6673 \pi$ & 306.217484 \\
\hline
\end{tabular}

\begin{tabular}{|l|c|c|c|c|c|c|c|}
\multicolumn{1}{|c|}{ Initial Clu ster Centers } \\
\hline & \multicolumn{7}{|c|}{ Cluster } \\
\cline { 2 - 8 } & 1 & 2 & 3 & 4 & 5 & 6 & 7 \\
\hline $\begin{array}{l}\text { Length width } \\
\text { Ratio }\end{array}$ & 2.491228 & 1.784404 & 1.017065 & 2.039409 & 1.491857 & 2.192308 & 1.206790 \\
\hline
\end{tabular}

\begin{tabular}{|l|c|c|c|c|c|c|c|}
\hline \multicolumn{1}{|c|}{ Initial Cluster Centers } \\
\cline { 2 - 8 } & \multicolumn{7}{|c|}{ Cluster } \\
\hline $\begin{array}{l}\text { Length width } \\
\text { Ratio }\end{array}$ & 1.414894 & 2.821656 & 2.319797 & 1.306569 & 1.650655 & 1.577061 & 1.926471 \\
\hline
\end{tabular}

\begin{tabular}{|c|c|c|c|c|c|c|c|c|}
\hline \multicolumn{10}{|c|}{ Initial Cluster Centers } \\
\hline & \multicolumn{10}{|c|}{ Cluster } \\
\cline { 2 - 9 } & 1 & 2 & 3 & 4 & 5 & 6 & 7 & 8 \\
\hline Area & 175316 & 207688 & 186587 & 240592 & 292808 & 269369 & 200254 & 227813 \\
\hline
\end{tabular}

\begin{tabular}{|c|c|c|c|c|c|c|c|c|}
\hline & \multicolumn{8}{|c|}{ Cluster } \\
\hline & 9 & \multicolumn{2}{|c|}{10} & 11 & 12 & \multicolumn{2}{|c|}{13} & 14 \\
\hline Area & 248800 & \multicolumn{2}{|c|}{134572} & 256387 & 193219 & \multicolumn{2}{|c|}{160739} & 213099 \\
\hline \multicolumn{9}{|c|}{ Initial Cluster Centers } \\
\hline & \multicolumn{8}{|c|}{ Cluster } \\
\hline & 1 & 2 & 3 & 4 & 5 & 6 & 7 & 8 \\
\hline Perimeter & 4396 & 4808 & 4946 & 5793 & 5720 & 6427 & 5205 & 4603 \\
\hline
\end{tabular}

Initial Cluster Centers

\begin{tabular}{|c|r|r|r|r|r|r|}
\hline & \multicolumn{6}{|c|}{ Cluster } \\
\cline { 2 - 7 } & \multicolumn{1}{|c|}{9} & \multicolumn{1}{|c|}{10} & \multicolumn{1}{|c|}{11} & \multicolumn{1}{c|}{12} & \multicolumn{1}{c|}{13} & \multicolumn{1}{c|}{14} \\
\hline Perimeter & 5056 & 5475 & 3815 & 5319 & 4530 & 5569 \\
\hline
\end{tabular}

Init|a| Cluster Centers

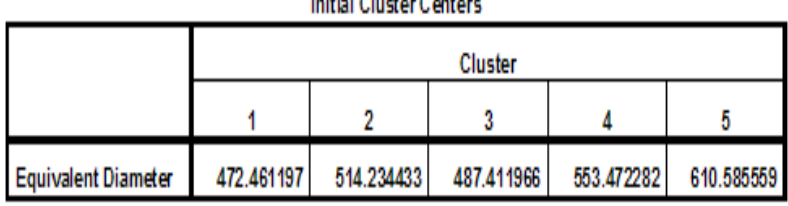

Initial Cluster Centers

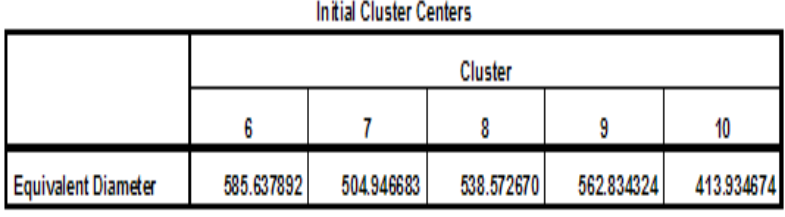

Intial Cluster Centers

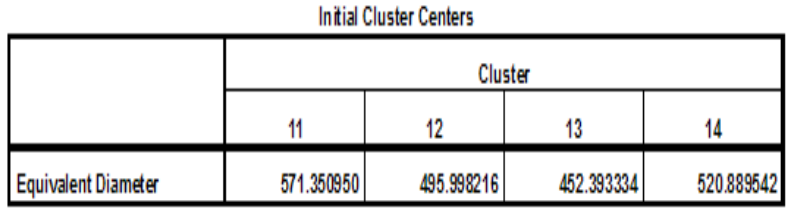

Initial Cluster Centers

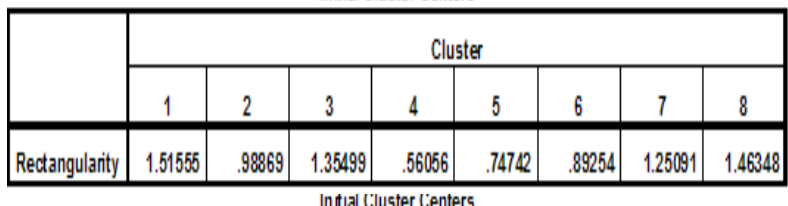

\begin{tabular}{|c|c|c|c|c|c|c|}
\hline \multirow{2}{*}{} & \multicolumn{7}{|c|}{ Cluster } \\
\cline { 2 - 7 } & 9 & 10 & 11 & 12 & 13 & 14 \\
\hline Rectangulanity & 1.06472 & 1.12193 & 1.58324 & 1.20264 & 2.47732 & 1.41198 \\
\hline
\end{tabular}
Initial Cluster Centers

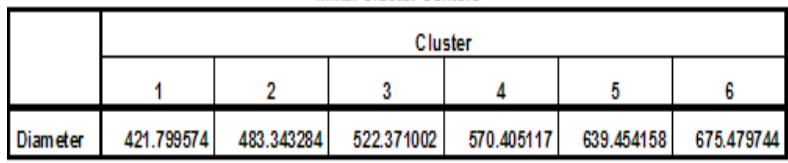
Intial Cluster Centers

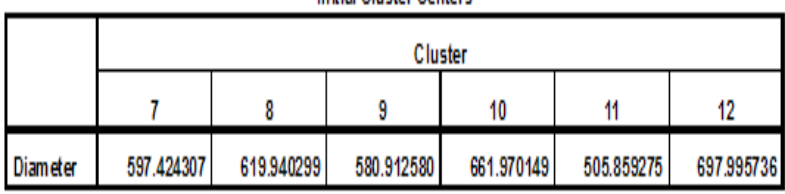
Initial Cluster Centers

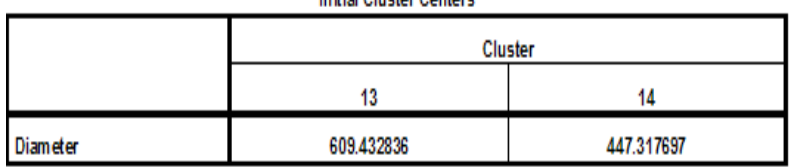

Initial Cluster Centers

\begin{tabular}{|l|c|c|c|c|c|c|}
\hline & \multicolumn{7}{|c|}{ Cluster } \\
\cline { 2 - 7 } & 1 & 2 & 3 & 4 & 5 & 6 \\
\hline $\begin{array}{l}\text { Perimeter Ratio of Branch } \\
\text { Length-Branch Width }\end{array}$ & 4.430537 & 4.718868 & 5.148424 & 4.235480 & 5.946467 & 5.038155 \\
\hline
\end{tabular}


International Journal of Computer Applications Technology and Research

Volume 2- Issue 5, 557 - 586, 2013, ISSN: 2319-8656

Initial Cluster Centers

\begin{tabular}{|c|c|c|c|c|c|c|}
\hline & \multicolumn{6}{|c|}{ Cluster } \\
\hline & 7 & 8 & 9 & 10 & 11 & 12 \\
\hline $\begin{array}{l}\text { Perimeter Ratio of Branch } \\
\text { Length-Branch Width }\end{array}$ & 4.880447 & 3.996112 & 5.745399 & 5.286098 & 6.160609 & 3.301852 \\
\hline \multicolumn{7}{|c|}{ Initial Cluster Centers } \\
\hline & & & \multicolumn{4}{|c|}{ Cluster } \\
\hline & & & \multicolumn{2}{|c|}{13} & \multicolumn{2}{|c|}{14} \\
\hline \multicolumn{3}{|c|}{ Perimeter Ratio of Branch Length-Branch Width } & & 5.415092 & \multicolumn{2}{|r|}{5.539335} \\
\hline
\end{tabular}

Initial Cluster Centers

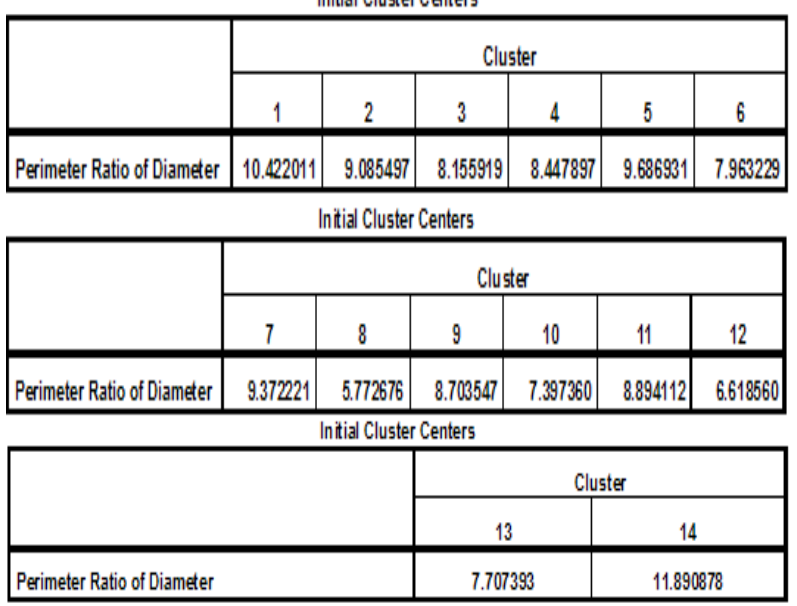

InitialCluster Centers

\begin{tabular}{|c|c|c|c|c|c|c|c|}
\hline & \multicolumn{7}{|c|}{ Cluster } \\
\cline { 2 - 8 } & 1 & 2 & 3 & 4 & 5 & 6 & 7 \\
\hline Convexity & 84.55687 & 79.52760 & 77.31115 & 72.58582 & 64.13240 & 57.83600 & 70.96449 \\
\hline
\end{tabular}

\begin{tabular}{|c|c|c|c|c|c|c|c|}
\hline & \multicolumn{7}{|c|}{ Cluster } \\
\cline { 2 - 8 } & 8 & 9 & 10 & 11 & 12 & 13 & 14 \\
\hline Convexity & 82.05563 & 80.74126 & 68.10407 & 66.74663 & 69.29754 & 87.08688 & 74.88155 \\
\hline
\end{tabular}

\begin{tabular}{|c|c|c|c|c|c|c|c|c|}
\hline \multirow{2}{*}{} & \multicolumn{7}{|c|}{ Cluster } \\
\cline { 2 - 9 } & 1 & 2 & 3 & 4 & 5 & 6 & 7 & 8 \\
\hline Solidity & .471645 & .558735 & .501968 & .647254 & .787728 & .724672 & .538734 & .612875 \\
\hline \multicolumn{8}{|c|}{ In inial Cluster Centers } \\
\hline
\end{tabular}

\begin{tabular}{|c|c|c|c|r|r|r|}
\hline \multirow{2}{*}{} & \multicolumn{7}{|c|}{ Cluster } \\
\cline { 2 - 7 } & 9 & 10 & 11 & 12 & 13 & 14 \\
\hline Solidity & .669336 & .400704 & .689746 & .519809 & .434213 & .573290 \\
\hline
\end{tabular}

\begin{tabular}{|c|c|c|c|c|c|c|c|c|}
\hline \multirow{10}{*}{} & \multicolumn{10}{|c|}{ Cluster } \\
\cline { 2 - 10 } & 1 & 2 & 3 & 4 & 5 & 6 & 7 & 8 \\
\hline On-Pixels & 175227 & 207604 & 186498 & 240498 & 292731 & 269295 & 200173 & 227711 \\
\hline \multicolumn{10}{|c|}{ Intial Cluster Centers } \\
\hline & \multicolumn{8}{|c|}{ Cluster } \\
\cline { 2 - 9 } & 9 & 10 & 11 & 12 & 13 & 14 \\
\hline On-Pixels & 248725 & 134514 & 256313 & 193144 & 160675 & 212979 \\
\hline
\end{tabular}

Initial Cluster Centers

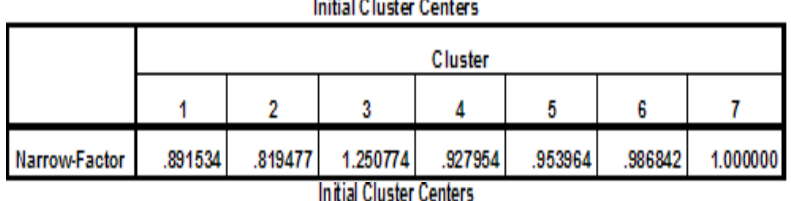

Intial Cluster Centers

\begin{tabular}{|c|c|c|c|c|c|c|c|}
\hline & \multicolumn{7}{|c|}{ Cluster } \\
\cline { 2 - 9 } & 8 & 9 & 10 & 11 & 12 & 13 & 14 \\
\hline Narrow.Factor & 1.146341 & 1.015284 & 1.065491 & 1.038560 & .974359 & 1.094017 & 1.111959 \\
\hline
\end{tabular}

Table 3 and Table 4 represent the iteration history of the $\mathrm{K}$ Means clustering on individual leaf and fruit features respectively. Iteration history shows the number of times the clustering iterates before completion. Clustering algorithm completes when there is a small change or no change in the cluster centers, there by achieving the convergence.

Table 3- Iteration History of clustering on 12 individual leaf features

Table 3.1- Iteration History of clustering on Major Axis

\begin{tabular}{|c|c|c|c|c|c|c|c|c|}
\hline & & & & on Histor & & & & \\
\hline \multirow[t]{2}{*}{ Iterstion } & \multicolumn{8}{|c|}{ Change in Cluster Centers } \\
\hline & 1 & 2 & 3 & 4 & 5 & 6 & 7 & 8 \\
\hline 1 & .000 & .000 & 2.150 & .000 & .000 & .000 & .530 & 2.502 \\
\hline 2 & .000 & .000 & .000 & .000 & .000 & .000 & .000 & .000 \\
\hline 3 & .000 & .000 & .000 & .000 & .000 & .000 & .000 & .000 \\
\hline
\end{tabular}

\begin{tabular}{|l|r|r|r|r|r|r|r|}
\hline \multirow{2}{*}{ Iteration } & \multicolumn{7}{|c|}{ Change in Cluster Centers } \\
\cline { 2 - 8 } & \multicolumn{1}{|c|}{9} & \multicolumn{1}{|c|}{10} & \multicolumn{1}{c|}{11} & \multicolumn{1}{c|}{12} & \multicolumn{1}{c|}{13} & \multicolumn{1}{c|}{14} & \multicolumn{1}{c|}{15} \\
\hline 1 & .000 & 3.002 & .000 & 4.203 & 4.003 & .000 & $4.000 \mathrm{E}-007$ \\
2 & .000 & 1.128 & .000 & .000 & .000 & .000 & 1.334 \\
3 & .000 & .000 & .000 & .000 & .000 & .000 & .000 \\
\hline
\end{tabular}

a. Convergence achieved due to no or small change in cluster centers. The maximum absolute coordinate change for any center is .000 . The current iteration is 3 . The minimum distance between initial centers is 12.009 .

Table 3.2- Iteration History of clustering on Minor Axis

\begin{tabular}{|c|c|c|c|c|c|c|c|c|}
\hline \multirow[t]{2}{*}{ Iteration } & \multicolumn{8}{|c|}{ Change in Cluster Centers } \\
\hline & 1 & 2 & 3 & 4 & 5 & 6 & 7 & 8 \\
\hline 1 & .375 & .000 & .790 & .481 & 1.501 & .375 & 1.001 & .000 \\
\hline 2 & 2.377 & .000 & .000 & .000 & .000 & .000 & .000 & .000 \\
\hline 3 & .000 & .000 & .000 & .000 & .000 & .000 & .000 & .000 \\
\hline
\end{tabular}

\begin{tabular}{|l|r|r|r|r|r|r|r|}
\hline \multirow{2}{*}{ Itegstion } & \multicolumn{7}{|c|}{ Change in Clusta Centers } \\
\cline { 2 - 8 } & \multicolumn{1}{|c|}{9} & \multicolumn{1}{|c|}{10} & \multicolumn{1}{|c|}{11} & \multicolumn{1}{c|}{12} & \multicolumn{1}{c|}{14} & \multicolumn{1}{c|}{15} \\
\hline 1 & 3.753 & .000 & .000 & .000 & 3.002 & 1.878 & 3.002 \\
2 & 1.751 & .000 & .000 & .000 & .000 & 1.876 & 1.501 \\
3 & .000 & .000 & .000 & .000 & .000 & .000 & .000 \\
\hline
\end{tabular}

a. Convergence achieved due to no or small change in cluster centers. The maximum absolute coordinate change for any center is .000 . The current iteration is 3 . The minimum distance between initial centers is 9.006 .

Table 3.3- Iteration History of clustering on Aspect Ratio 


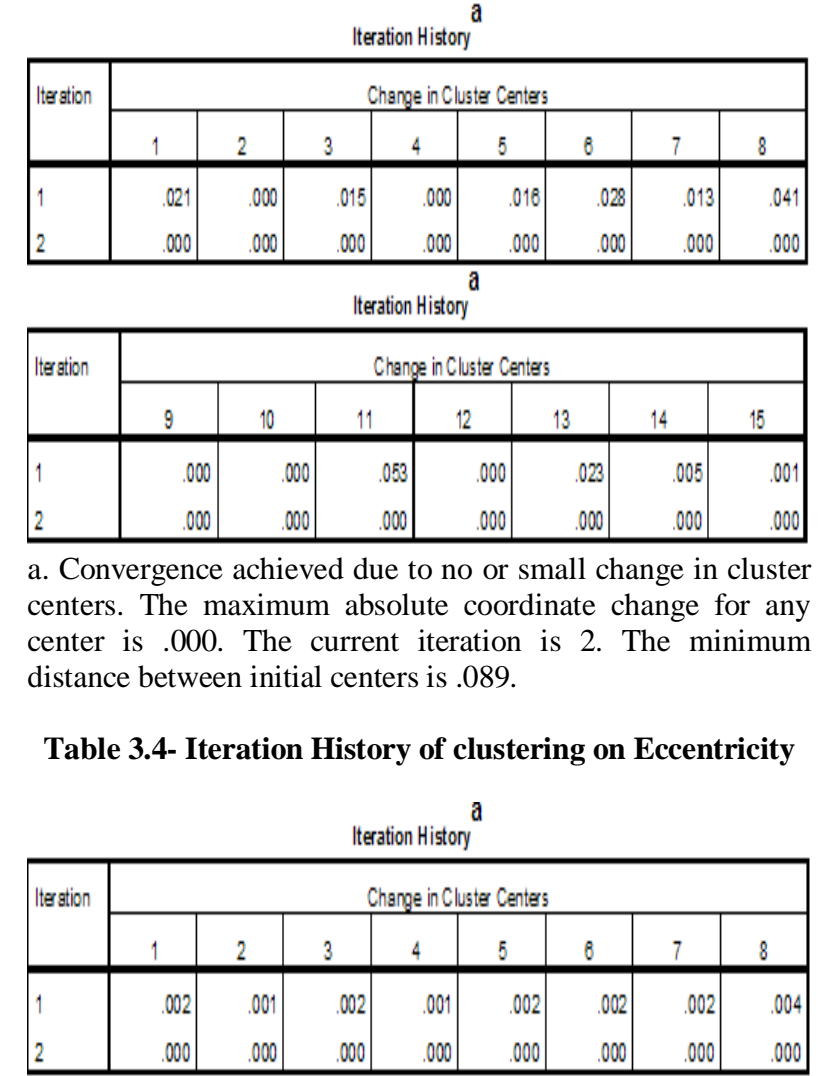

\begin{tabular}{|c|c|c|c|c|c|c|c|}
\hline & & & Iteratio & story & & & \\
\hline \multirow[t]{2}{*}{ Iteration } & \multicolumn{7}{|c|}{ Change in Cluster Centers } \\
\hline & 9 & 10 & 11 & 12 & 13 & 14 & 15 \\
\hline 1 & .002 & .000 & .000 & .000 & .003 & $2.325 \mathrm{E}-005$ & .000 \\
\hline 2 & .000 & .000 & .000 & .000 & .000 & .000 & .000 \\
\hline
\end{tabular}

a. Convergence achieved due to no or small change in cluster centers. The maximum absolute coordinate change for any center is .000. The current iteration is 2. The minimum distance between initial centers is .008 .

Table 3.5- Iteration History of clustering on Area

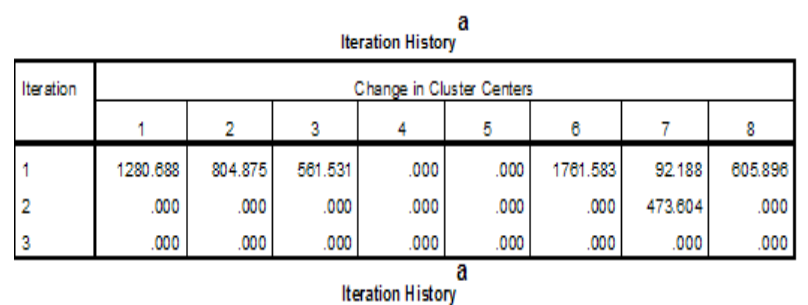

\begin{tabular}{|c|c|c|c|c|c|c|c|}
\hline \multirow[t]{2}{*}{ Itegation } & \multicolumn{7}{|c|}{ Change in Cluster Centers } \\
\hline & 9 & 10 & 11 & 12 & 13 & 14 & 15 \\
\hline 1 & 109.938 & .000 & 47.083 & .000 & .000 & 49.875 & 788.925 \\
\hline 2 & .000 & .000 & .000 & .000 & .000 & .000 & 513.044 \\
\hline 3 & .000 & .000 & .000 & .000 & .000 & .000 & .000 \\
\hline
\end{tabular}

a. Convergence achieved due to no or small change in cluster centers. The maximum absolute coordinate change for any center is .000 . The current iteration is 3 . The minimum distance between initial centers is 2776.250 .
Table 3.6- Iteration History of clustering on Rectangularity

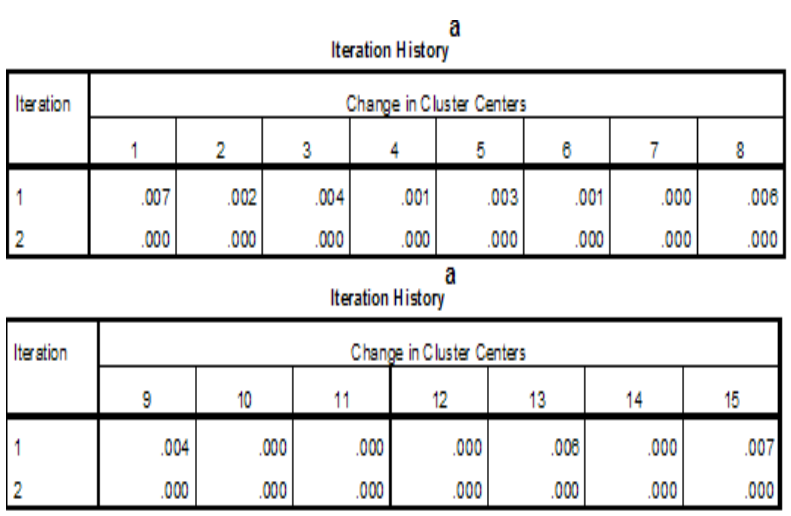

a. Convergence achieved due to no or small change in cluster centers. The maximum absolute coordinate change for any center is .000 . The current iteration is 2 . The minimum distance between initial centers is .020 .

Table 3.7- Iteration History of clustering on Diameter

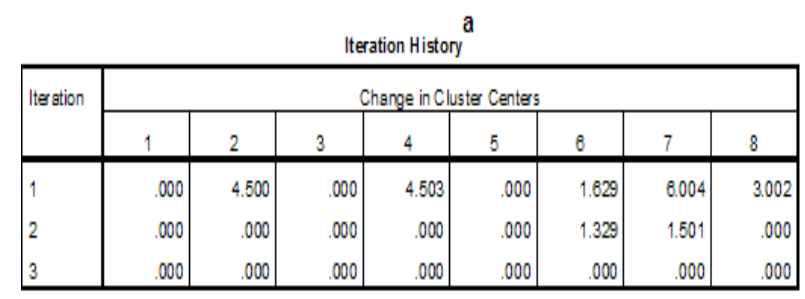

\begin{tabular}{|c|c|c|c|c|c|c|c|}
\hline & & & Iteration & & & & \\
\hline \multirow[t]{2}{*}{ literstion } & \multicolumn{7}{|c|}{ Change in Cluster Centers } \\
\hline & 9 & 10 & 11 & 12 & 13 & 14 & 15 \\
\hline 1 & .000 & .000 & 4.878 & 1.001 & .429 & .500 & 1.878 \\
\hline 2 & .000 & .000 & 1.128 & 4.003 & .000 & .000 & .000 \\
\hline 3 & .000 & .000 & .000 & .000 & .000 & .000 & .000 \\
\hline
\end{tabular}

a. Convergence achieved due to no or small change in cluster centers. The maximum absolute coordinate change for any center is .000 . The current iteration is 3 . The minimum distance between initial centers is 13.510 .

Table 3.8- Iteration History of clustering on Compactness
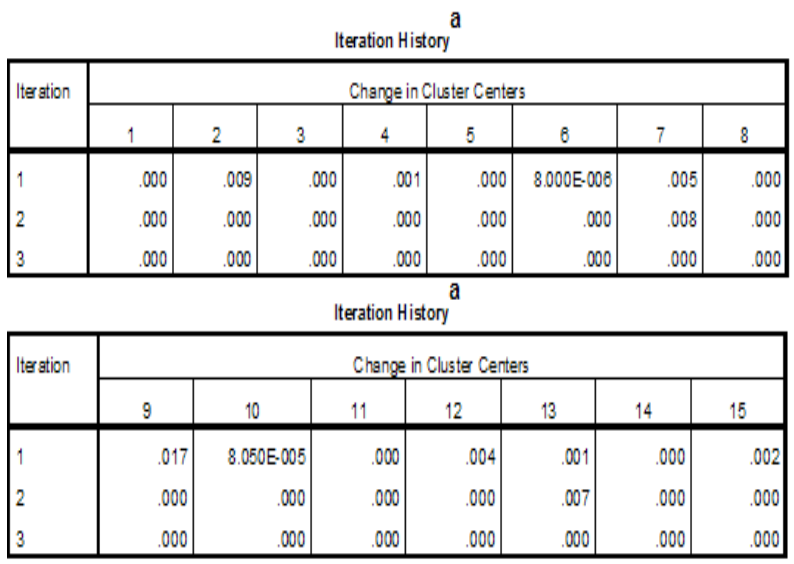

a. Convergence achieved due to no or small change in cluster centers. The maximum absolute coordinate change for any 
center is .000 . The current iteration is 3 . The minimum distance between initial centers is .025 .

Table 3.9- Iteration History of clustering on Perimeter Ratio of Major Axis-Minor Axis

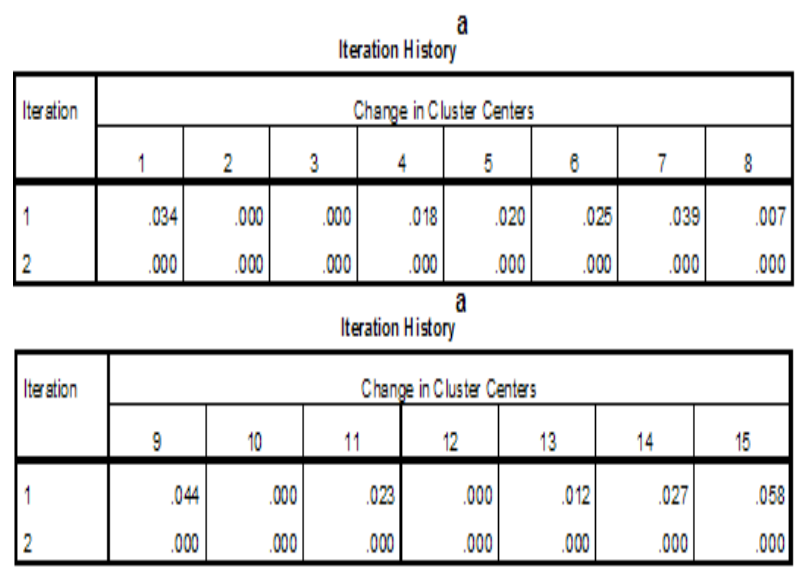

a. Convergence achieved due to no or small change in cluster centers. The maximum absolute coordinate change for any center is .000. The current iteration is 2 . The minimum distance between initial centers is .115 .

Table 3.10- Iteration History of clustering on Perimeter Ratio of Diameter

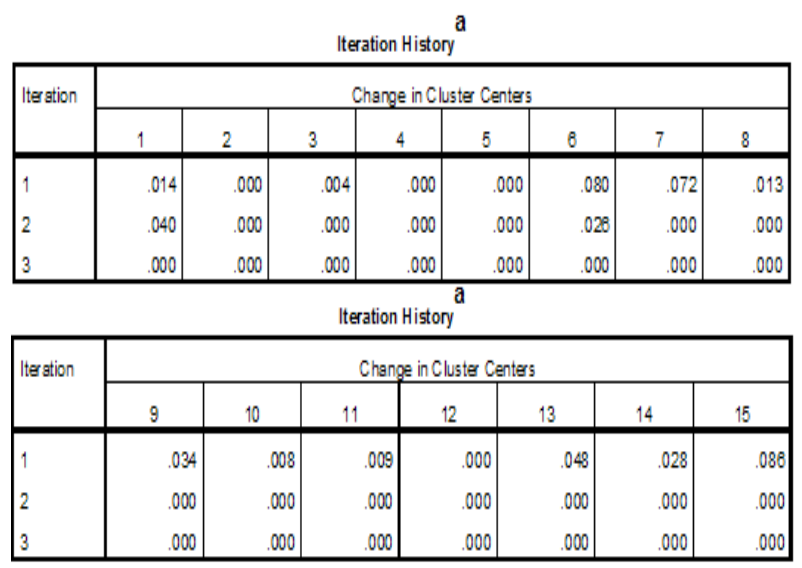

a. Convergence achieved due to no or small change in cluster centers. The maximum absolute coordinate change for any center is .000 . The current iteration is 3 . The minimum distance between initial centers is 237 .

Table 3.11- Iteration History of clustering on Concavity

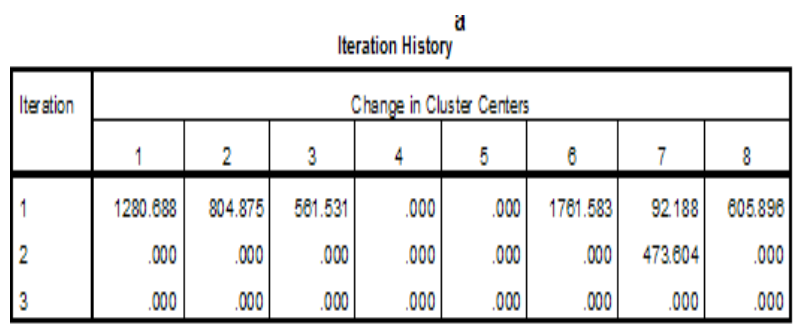

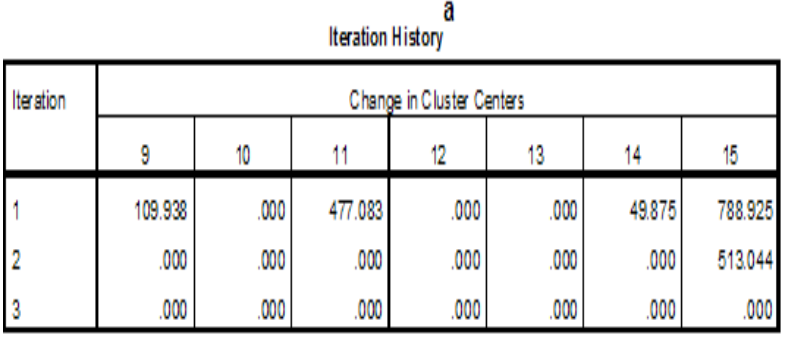

a. Convergence achieved due to no or small change in cluster centers. The maximum absolute coordinate change for any center is .000 . The current iteration is 3 . The minimum distance between initial centers is 2776.250 .

Table 3.12- Iteration History of clustering on R-Factor

\begin{tabular}{|c|c|c|c|c|c|c|c|}
\hline \multirow[t]{2}{*}{ Iter ation } & \multicolumn{7}{|c|}{ Change in Cluster Centers } \\
\hline & 1 & 2 & 3 & 5 & 8 & 7 & 8 \\
\hline 1 & .000 & .000 & .000 & .000 & 1.634 & 3.755 & .000 \\
\hline 2 & .000 & .000 & .000 & .000 & .000 & .000 & .000 \\
\hline 3 & .000 & .000 & .000 & .000 & .000 & .000 & .000 \\
\hline \multicolumn{8}{|c|}{ Iteration History } \\
\hline \multirow[t]{2}{*}{ Iteration } & \multicolumn{7}{|c|}{ Change in Cluster Centers } \\
\hline & 9 & 10 & 11 & 12 & 13 & 14 & 15 \\
\hline 1 & 3.273 & 1.387 & 7.984 & 8.423 & .802 & 3.522 & 4.450 \\
\hline 2 & 5.889 & .000 & 2.004 & .000 & .000 & .000 & .000 \\
\hline 3 & .000 & .000 & .000 & .000 & .000 & .000 & .000 \\
\hline
\end{tabular}

a. Convergence achieved due to no or small change in cluster centers. The maximum absolute coordinate change for any center is .000 . The current iteration is 3 . The minimum distance between initial centers is 19.686 .

Table 4- Iteration History of clustering on 14 individual fruit features

Table 4.1- Iteration History of clustering on BranchLength

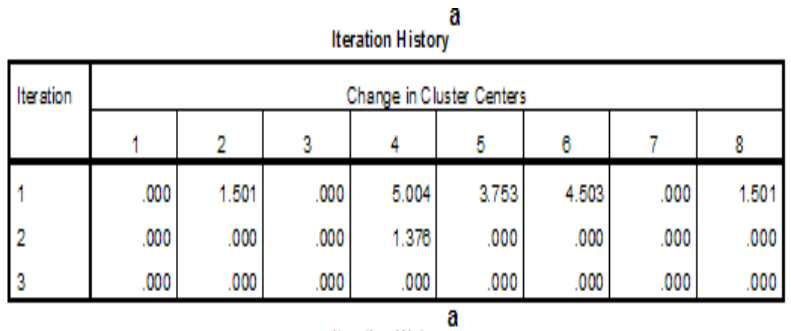

Iteration History

\begin{tabular}{|l|r|r|r|r|r|r|}
\hline \multirow{2}{*}{ Itergtion } & \multicolumn{6}{|c|}{ Change in Cluster Centers } \\
\cline { 2 - 7 } & \multicolumn{1}{|c|}{9} & \multicolumn{1}{|c|}{10} & \multicolumn{1}{c|}{11} & \multicolumn{1}{c|}{12} & \multicolumn{1}{c|}{13} & \multicolumn{1}{c|}{14} \\
\hline 1 & .503 & .000 & 2.502 & 3.002 & 3.002 & 3.002 \\
2 & .000 & .000 & .000 & .000 & .000 & 1.501 \\
3 & .000 & .000 & .000 & .000 & .000 & .000 \\
\hline
\end{tabular}

a. Convergence achieved due to no or small change in cluster centers. The maximum absolute coordinate change for any center is .000 . The current iteration is 3 . The minimum distance between initial centers is 12.009 .

Table 4.2- Iteration History of clustering on BranchWidth 


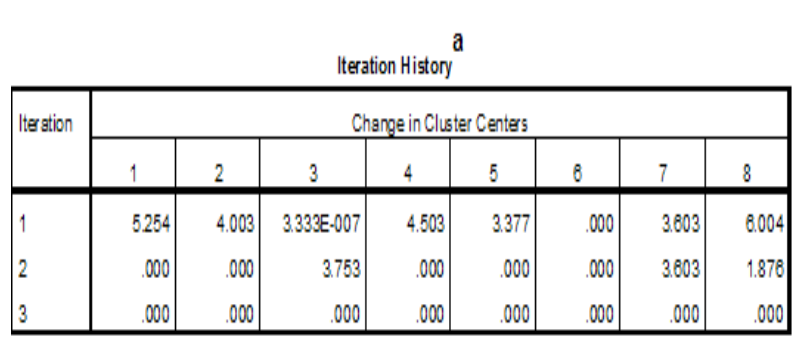

\begin{tabular}{|c|c|c|c|c|c|c|}
\hline \multirow{3}{*}{ Itegation } & \multicolumn{6}{|c|}{ Iteration History } \\
\hline & \multicolumn{6}{|c|}{ Change in Clusta Centers } \\
\hline & 9 & 10 & 11 & 12 & 13 & 14 \\
\hline 1 & 1.501 & .000 & .751 & 3.377 & .000 & 1.501 \\
\hline 2 & 1.501 & .000 & 1.951 & .000 & .000 & .000 \\
\hline 3 & .000 & .000 & .000 & .000 & .000 & .000 \\
\hline
\end{tabular}

a. Convergence achieved due to no or small change in cluster centers. The maximum absolute coordinate change for any center is .000 . The current iteration is 3 . The minimum distance between initial centers is 10.507 .

Table 4.3- Iteration History of clustering on LengthWidth Ratio

\begin{tabular}{|c|c|c|c|c|c|c|c|c|}
\hline \multirow[t]{2}{*}{ Iterstion } & \multicolumn{8}{|c|}{ Change in Cluster Centers } \\
\hline & 1 & 2 & 3 & 4 & 5 & 8 & 7 & 8 \\
\hline 1 & .000 & .021 & .013 & .004 & .010 & .000 & .025 & .023 \\
\hline 2 & .000 & .000 & .000 & .000 & .000 & .000 & .007 & .000 \\
\hline 3 & .000 & .000 & .000 & .000 & .000 & .000 & .000 & .000 \\
\hline
\end{tabular}

\begin{tabular}{|l|r|r|r|r|r|r|}
\hline \multirow{2}{*}{ Itegstion } & \multicolumn{7}{|c|}{ Change in Clustar Centers } \\
\cline { 2 - 7 } & \multicolumn{1}{|c|}{9} & \multicolumn{1}{|c|}{10} & \multicolumn{1}{|c|}{11} & \multicolumn{1}{c|}{12} & \multicolumn{1}{c|}{13} & \multicolumn{1}{c|}{14} \\
\hline 1 & .000 & .000 & .019 & .004 & .018 & .021 \\
2 & .000 & .000 & .009 & .000 & .000 & .000 \\
3 & .000 & .000 & .000 & .000 & .000 & .000 \\
\hline
\end{tabular}

a. Convergence achieved due to no or small change in cluster centers. The maximum absolute coordinate change for any center is .000 . The current iteration is 3 . The minimum distance between initial centers is .074 .

Table 4.4- Iteration History of clustering on Area

\begin{tabular}{|c|c|c|c|c|c|c|c|c|}
\hline & & & & tion Histo & & & & \\
\hline \multirow[t]{2}{*}{ Itegation } & \multicolumn{8}{|c|}{ Change in Cluster Centers } \\
\hline & 1 & 2 & 3 & 4 & 5 & 8 & 7 & 8 \\
\hline 1 & 249.813 & 420.825 & 1882792 & 1128.833 & .000 & 2781.417 & .000 & 2080.188 \\
\hline 2 & .000 & .000 & .000 & .000 & .000 & .000 & .000 & .000 \\
\hline
\end{tabular}

\begin{tabular}{|c|c|c|c|c|c|c|}
\hline \multirow[t]{2}{*}{ litestion } & \multicolumn{6}{|c|}{ Change in Cluster Centers } \\
\hline & 9 & 10 & 11 & 12 & 13 & 14 \\
\hline 1 & 529.025 & .000 & 1237.800 & .000 & 331.438 & 2533.313 \\
\hline 2 & .000 & .000 & .000 & .000 & .000 & .000 \\
\hline
\end{tabular}

a. Convergence achieved due to no or small change in cluster centers. The maximum absolute coordinate change for any center is .000 . The current iteration is 2 . The minimum distance between initial centers is 5410.500 .

Table 4.5- Iteration History of clustering on Perimeter

\begin{tabular}{|c|c|c|c|c|c|c|c|c|}
\hline & & & & in Histo & & & & \\
\hline \multirow[t]{2}{*}{ Itergation } & \multicolumn{8}{|c|}{ Change in Cluster Centers } \\
\hline & 1 & 2 & 3 & 4 & 5 & 8 & 7 & 8 \\
\hline 1 & .000 & 31.000 & 9.000 & .000 & .000 & .000 & 2889 & 37.500 \\
\hline 2 & .000 & .000 & .000 & .000 & .000 & .000 & 8.488 & .000 \\
\hline 3 & .000 & .000 & .000 & .000 & .000 & .000 & .000 & .000 \\
\hline
\end{tabular}

\begin{tabular}{|l|r|r|r|r|r|r|}
\hline \multirow{2}{*}{ litgation } & \multicolumn{6}{|c|}{ Change in Cluster Centers } \\
\cline { 2 - 7 } & \multicolumn{1}{|c|}{9} & \multicolumn{1}{|c|}{10} & \multicolumn{1}{c|}{11} & \multicolumn{1}{c|}{12} & \multicolumn{1}{c|}{13} & \multicolumn{1}{c|}{14} \\
\hline 1 & 24.333 & 4.807 & 20.500 & 10.444 & 15.000 & 7.500 \\
2 & 14.917 & .000 & .000 & .000 & .000 & .000 \\
3 & .000 & .000 & .000 & .000 & .000 & .000 \\
\hline
\end{tabular}

a. Convergence achieved due to no or small change in cluster centers. The maximum absolute coordinate change for any center is .000 . The current iteration is 3 . The minimum distance between initial centers is 73.000 .

Table 4.6- Iteration History of clustering on Equivalent-

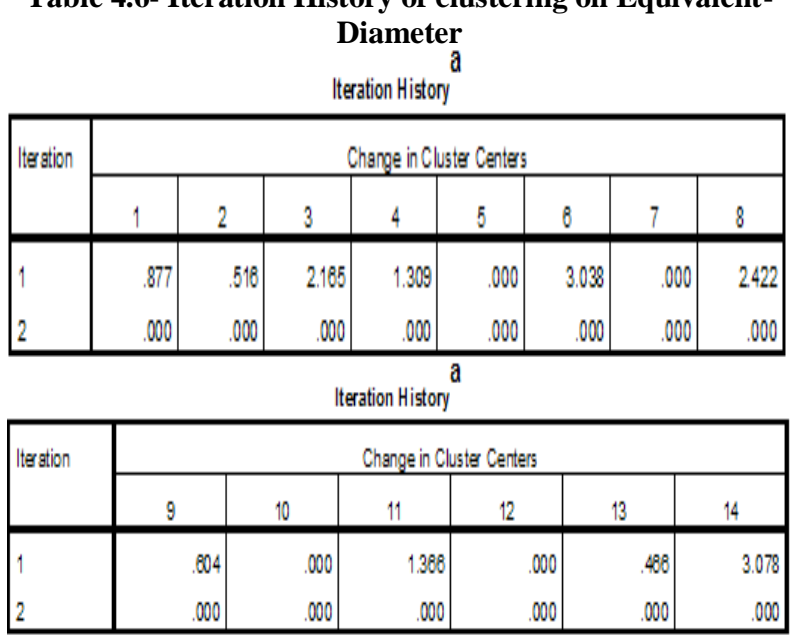

a. Convergence achieved due to no or small change in cluster centers. The maximum absolute coordinate change for any center is .000 . The current iteration is 2 . The minimum distance between initial centers is 6.655 .

Table 4.7- Iteration History of clustering on Rectangularity 


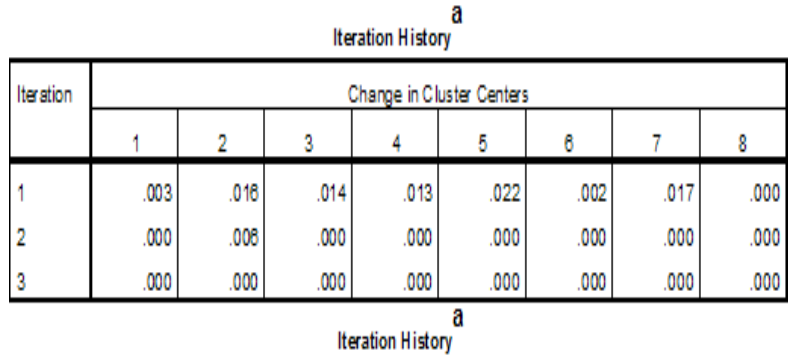

\begin{tabular}{|l|r|r|r|r|r|r|}
\hline \multirow{2}{*}{ Itegstion } & \multicolumn{7}{|c|}{ Change in Cluster Centers } \\
\cline { 2 - 7 } & \multicolumn{1}{|c|}{9} & \multicolumn{1}{|c|}{10} & \multicolumn{1}{c|}{11} & \multicolumn{1}{c|}{12} & \multicolumn{1}{c|}{13} & \multicolumn{1}{c|}{14} \\
\hline 1 & .004 & .012 & .008 & .004 & .000 & .001 \\
2 & .012 & .000 & .000 & .000 & .000 & .000 \\
3 & .000 & .000 & .000 & .000 & .000 & .000 \\
\hline
\end{tabular}

a. Convergence achieved due to no or small change in cluster centers. The maximum absolute coordinate change for any center is .000 . The current iteration is 3 . The minimum distance between initial centers is .048.

Table 4.8- Iteration History of clustering on Diameter

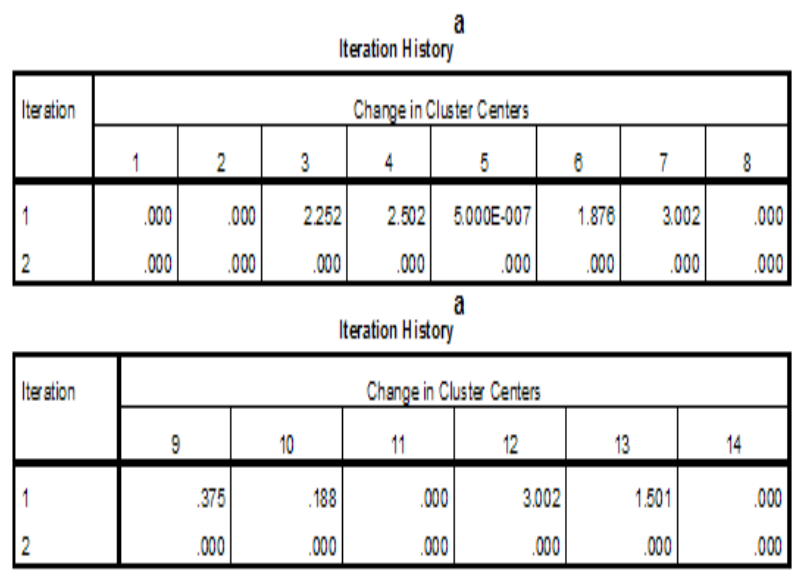

a. Convergence achieved due to no or small change in cluster centers. The maximum absolute coordinate change for any center is .000 . The current iteration is 2 . The minimum distance between initial centers is 10.507 .

Table 4.9- Iteration History of clustering on Perimeter Ratio of Branch Length-Branch Width

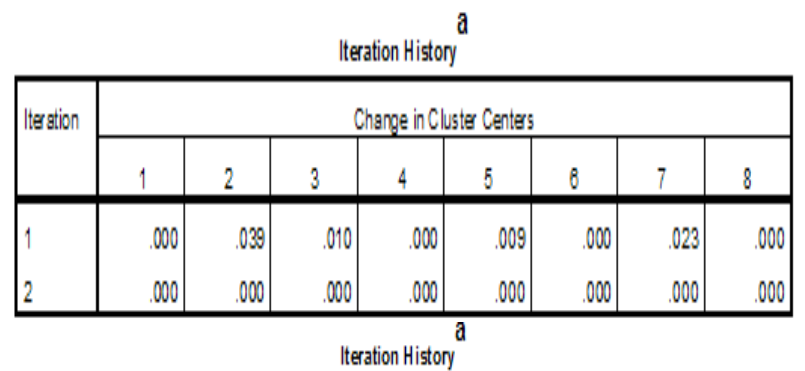

\begin{tabular}{|l|r|r|r|r|r|r|}
\hline \multirow{2}{*}{ Itegstion } & \multicolumn{6}{|c|}{ Change in Cluster Centers } \\
\cline { 2 - 7 } & \multicolumn{1}{|c|}{1} & \multicolumn{1}{|c|}{10} & \multicolumn{1}{|c|}{11} & \multicolumn{1}{c|}{12} & \multicolumn{1}{c|}{13} & \multicolumn{1}{c|}{14} \\
\hline 1 & .007 & .024 & .024 & .000 & .013 & .000 \\
2 & .000 & .000 & .000 & .000 & .000 & .000 \\
\hline
\end{tabular}

a. Convergence achieved due to no or small change in cluster centers. The maximum absolute coordinate change for any center is .000 . The current iteration is 2 . The minimum distance between initial centers is .110 .

Table 4.10- Iteration History of clustering on Perimeter Ratio of Diameter

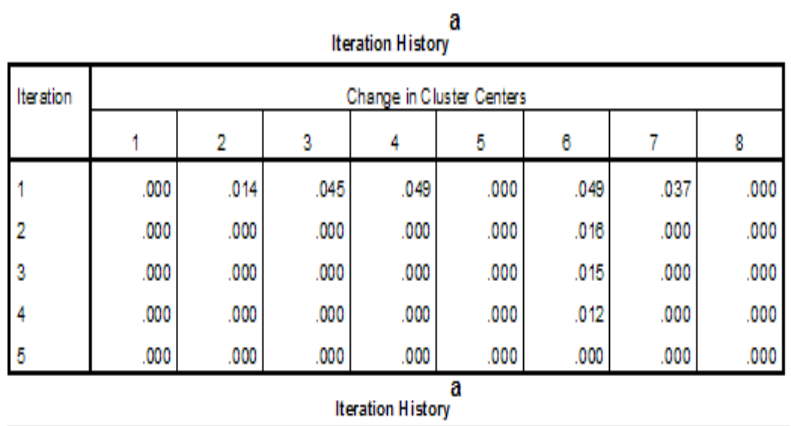

\begin{tabular}{|l|r|r|r|r|r|r|}
\hline \multirow{2}{*}{ tes ation } & \multicolumn{6}{|c|}{ Change in Cluster Centers } \\
\cline { 2 - 7 } & \multicolumn{1}{|c|}{9} & \multicolumn{1}{|c|}{10} & \multicolumn{1}{c|}{11} & \multicolumn{1}{c|}{12} & \multicolumn{1}{l|}{13} & \multicolumn{1}{c|}{14} \\
\hline 1 & .018 & .014 & .015 & .000 & .009 & .000 \\
2 & .000 & .000 & .000 & .000 & .015 & .000 \\
3 & .000 & .000 & .000 & .000 & .018 & .000 \\
4 & .000 & .000 & .000 & .000 & .021 & .000 \\
4 & .000 & .000 & .000 & .000 & .000 & .000 \\
5 & & &
\end{tabular}

a. Convergence achieved due to no or small change in cluster centers. The maximum absolute coordinate change for any center is .000 . The current iteration is 5. The minimum distance between initial centers is .191.

Table 4.11- Iteration History of clustering on Convexity

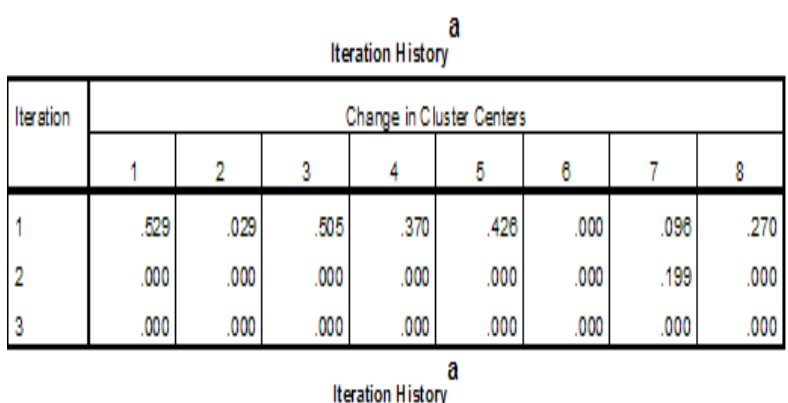

\begin{tabular}{|l|r|r|r|r|r|r|}
\multicolumn{7}{|c|}{ Iteration History } \\
\hline \multirow{2}{*}{ Itegstion } & \multicolumn{7}{|c|}{ Change in Cluster Centers } \\
\cline { 2 - 7 } & \multicolumn{1}{|c|}{1} & \multicolumn{1}{|c|}{10} & \multicolumn{1}{c|}{11} & \multicolumn{1}{c|}{12} & \multicolumn{1}{c|}{13} & \multicolumn{1}{c|}{14} \\
\hline 1 & .055 & .288 & .090 & .302 & .000 & .138 \\
2 & .000 & .000 & .000 & .148 & .000 & .000 \\
3 & .000 & .000 & .000 & .000 & .000 & .000 \\
\hline
\end{tabular}

a. Convergence achieved due to no or small change in cluster centers. The maximum absolute coordinate change for any center is .000 . The current iteration is 3 . The minimum distance between initial centers is 1.193 .

Table 4.12- Iteration History of clustering on Solidity 


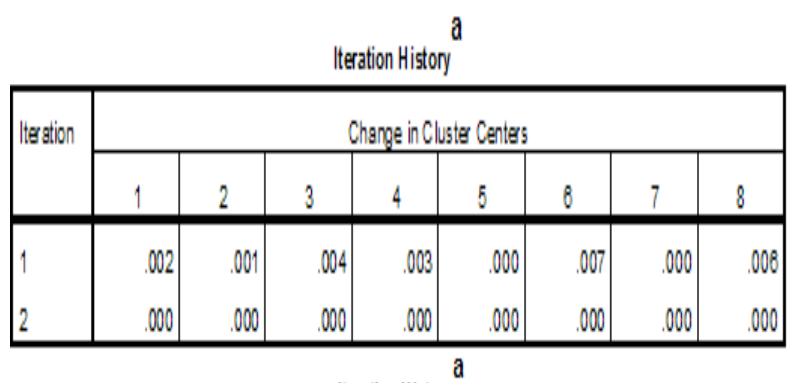

Iteration History

\begin{tabular}{|l|r|r|r|r|r|r|}
\hline \multirow{2}{*}{ Itegsion } & \multicolumn{6}{|c|}{ Change in Cluster Centers } \\
\cline { 2 - 7 } & \multicolumn{1}{|c|}{9} & \multicolumn{1}{|c|}{10} & \multicolumn{1}{c|}{11} & \multicolumn{1}{c|}{12} & \multicolumn{1}{c|}{13} & \multicolumn{1}{c|}{14} \\
\hline 1 & .001 & .000 & .003 & .000 & .000 & .007 \\
2 & .000 & .000 & .000 & .000 & .000 & .000 \\
\hline
\end{tabular}

a. Convergence achieved due to no or small change in cluster centers. The maximum absolute coordinate change for any center is .000. The current iteration is 2 . The minimum distance between initial centers is .015 .

Table 4.13- Iteration History of clustering on On Pixels

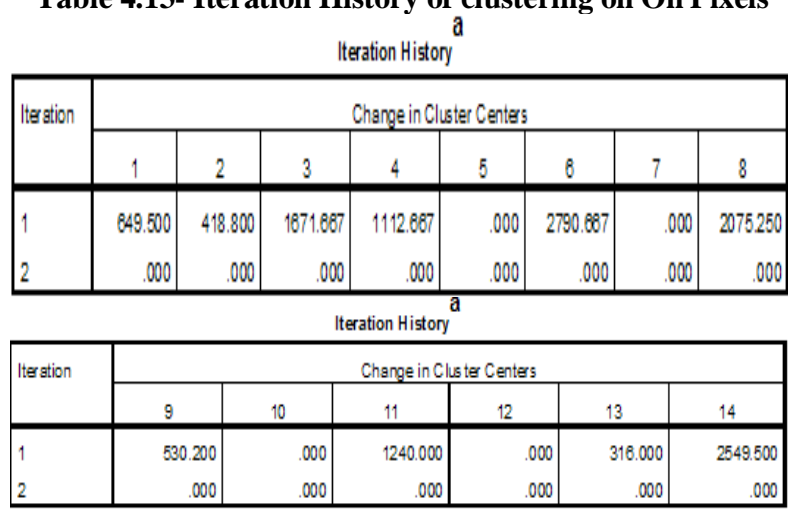

a. Convergence achieved due to no or small change in cluster centers. The maximum absolute coordinate change for any center is .000 . The current iteration is 2 . The minimum distance between initial centers is 5375.000 .

Table 4.14- Iteration History of clustering on NarrowFactor

\begin{tabular}{|c|c|c|c|c|c|c|c|c|}
\hline \multirow[t]{2}{*}{ Iteration } & \multicolumn{8}{|c|}{ Change in Cluster Centers } \\
\hline & 1 & 2 & 3 & 4 & 5 & 8 & 7 & 8 \\
\hline 1 & .000 & .005 & .000 & .001 & .000 & .000 & .001 & .005 \\
\hline 2 & .000 & .000 & .000 & .000 & .000 & .000 & .000 & .000 \\
\hline
\end{tabular}

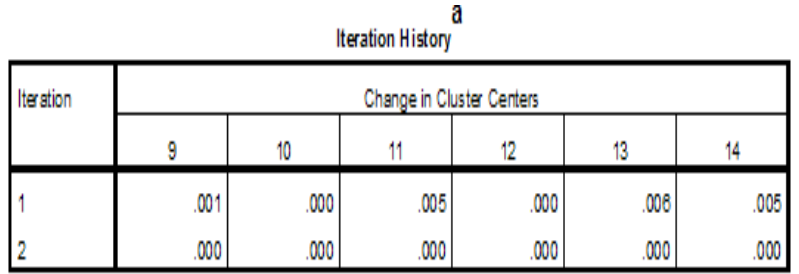

a. Convergence achieved due to no or small change in cluster centers. The maximum absolute coordinate change for any center is .000 . The current iteration is 2 . The minimum distance between initial centers is .012 .

Information regarding the membership of 15 leaf and 14 fruit clusters build through K-Means Clustering is shown by Table-
5 and Table 6. The case number field signifies the sample leaf/fruit pattern number. The field called cluster is the cluster number in which a pattern is placed and distance field gives the distance between the pattern and the cluster center, in which the pattern is placed.

Table 5-Cluster membership of leaf clusters build from 12 individual leaf features

Table 5.1-Cluster membership for Major Axis

\begin{tabular}{|c|c|c|c|c|c|}
\hline \multicolumn{3}{|c|}{ Cluster Membership } & \multicolumn{3}{|c|}{ Cluster Membership } \\
\hline $\begin{array}{l}\text { Case } \\
\text { Number }\end{array}$ & Cluster & Distance & $\begin{array}{l}\text { Case } \\
\text { Number }\end{array}$ & \begin{tabular}{|l|} 
Case \\
Number
\end{tabular} & \begin{tabular}{|l} 
Case \\
Number
\end{tabular} \\
\hline 1 & 1 & .000 & 16 & 10 & .375 \\
\hline 2 & 2 & .000 & 17 & 15 & 7.339 \\
\hline 3 & 3 & 2.150 & 18 & 13 & 1.001 \\
\hline 4 & 4 & .000 & 19 & 15 & 4.670 \\
\hline 5 & 5 & .000 & 20 & 12 & 1.201 \\
\hline 6 & 6 & .000 & 21 & 10 & 7.881 \\
\hline 7 & 7 & 1.148 & 22 & 8 & 1.001 \\
\hline 8 & 15 & 1.334 & 23 & 10 & 4.128 \\
\hline 9 & 9 & .000 & 24 & 15 & 1.668 \\
\hline 10 & 10 & 1.126 & 25 & 15 & .167 \\
\hline 11 & 7 & .530 & 26 & 11 & .000 \\
\hline 12 & 12 & 4.203 & 27 & 10 & 4.878 \\
\hline 13 & 15 & 1.334 & 28 & 8 & 2.502 \\
\hline 14 & 7 & 2.031 & 29 & 7 & .530 \\
\hline 15 & 15 & 1.668 & 30 & 12 & 4.803 \\
\hline
\end{tabular}

\begin{tabular}{|l|r|r|}
\hline \multicolumn{3}{|c|}{ Cluster Membership } \\
\hline $\begin{array}{l}\text { Case } \\
\text { Number }\end{array}$ & Cluster & Distance \\
\hline 31 & 11 & .000 \\
\hline 32 & 13 & 4.003 \\
\hline 33 & 7 & 2.472 \\
\hline 34 & 12 & 4.203 \\
\hline 35 & 7 & .530 \\
\hline 36 & 10 & 5.629 \\
\hline 37 & 10 & 6.380 \\
\hline 38 & 15 & 3.169 \\
\hline 39 & 14 & .000 \\
\hline 40 & 3 & 2.150 \\
\hline 41 & 12 & 4.803 \\
\hline 42 & 10 & 8.631 \\
\hline 43 & 8 & 3.502 \\
\hline 44 & 15 & 1.334 \\
\hline 45 & 13 & 5.004 \\
\hline
\end{tabular}

Table 5.2-Cluster membership for Minor Axis 


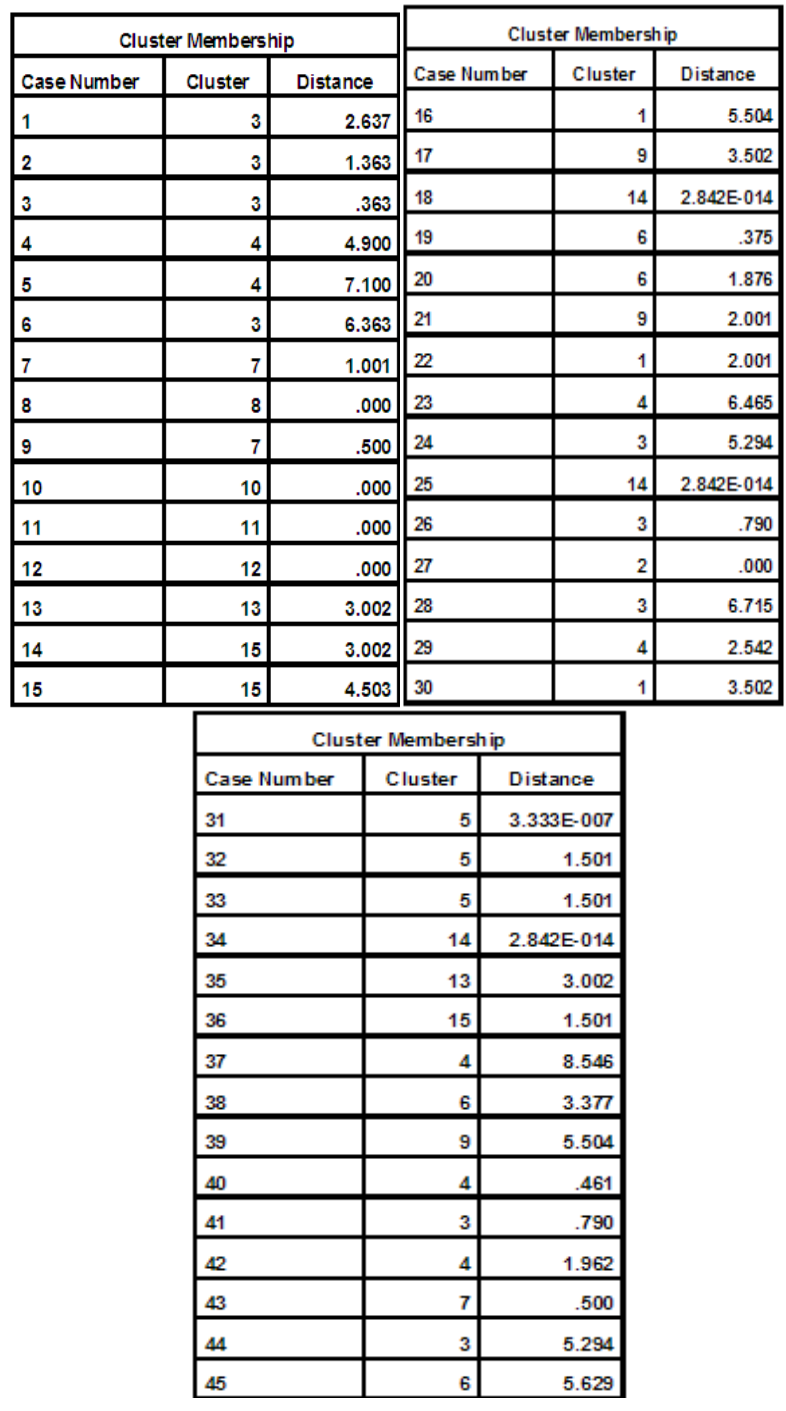

Table 5.3-Cluster membership for Aspect Ratio

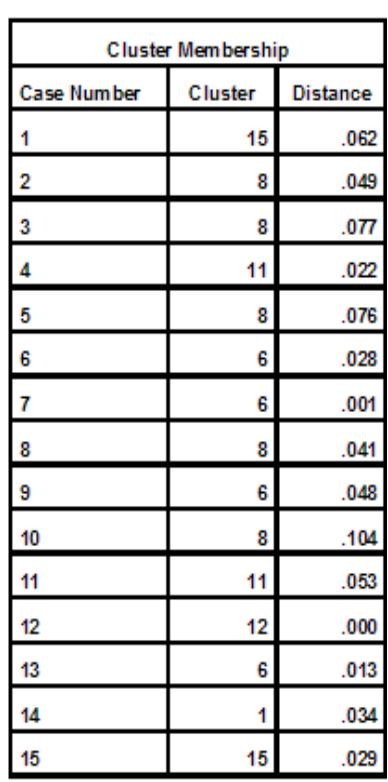

\begin{tabular}{|l|r|r|}
\hline \multicolumn{3}{|c|}{ Cluster Membership } \\
\hline Case Number & Cluster & Distance \\
\hline 16 & 3 & .015 \\
\hline 17 & 7 & .013 \\
\hline 18 & 13 & .028 \\
\hline 19 & 3 & .028 \\
\hline 20 & 5 & .016 \\
\hline 21 & 7 & .008 \\
\hline 22 & 3 & .044 \\
\hline 23 & 2 & .000 \\
\hline 24 & 1 & .035 \\
\hline 25 & 14 & .017 \\
\hline 26 & 14 & .012 \\
\hline 27 & 11 & .031 \\
\hline 28 & 14 & .005 \\
\hline 29 & 9 & .000 \\
\hline 30 & 7 & .022 \\
\hline
\end{tabular}

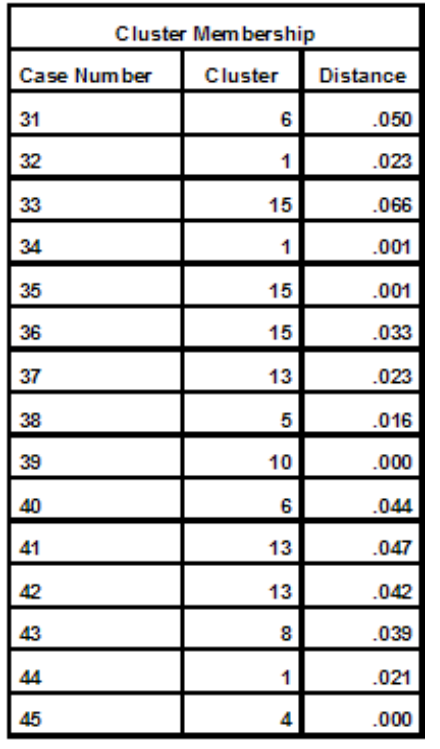

Table 5.4-Cluster membership for Eccentricity
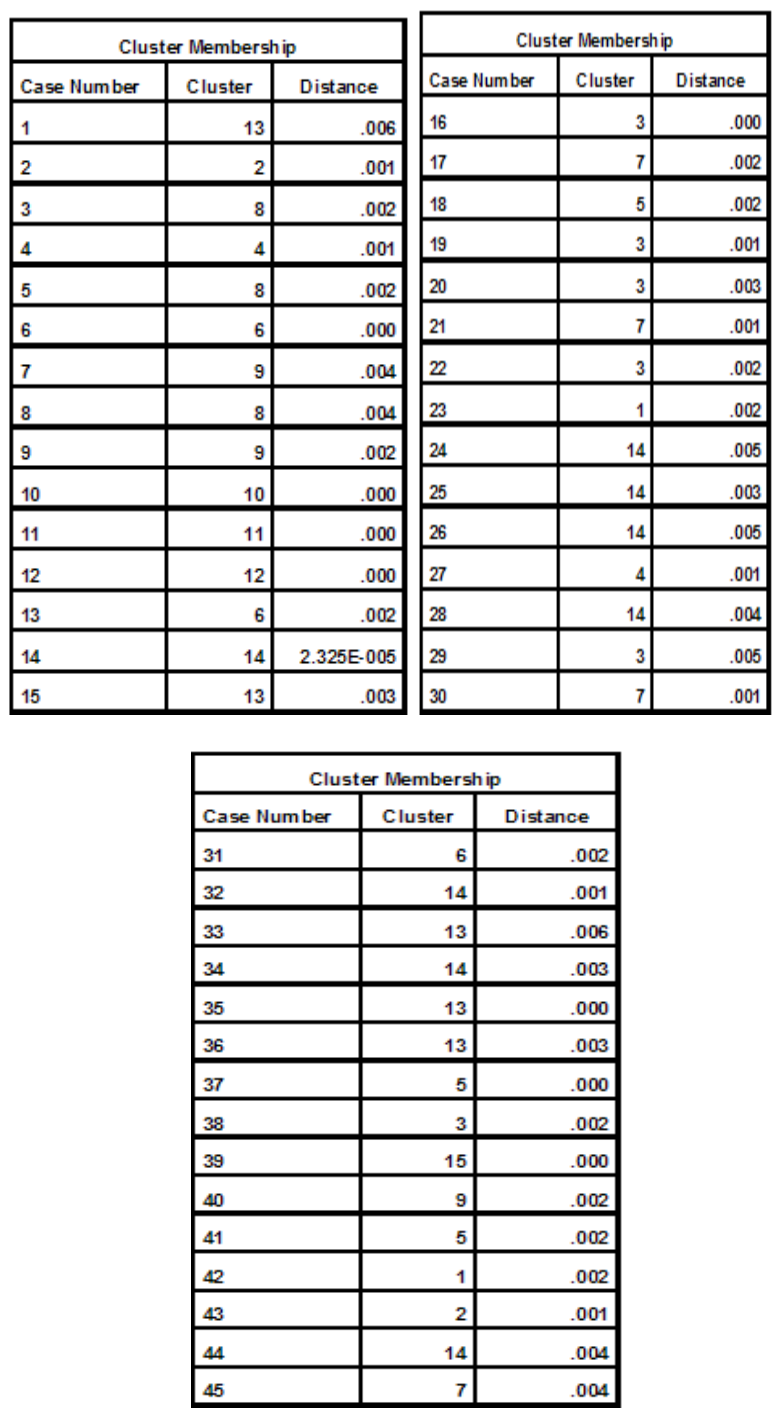

Table 5.5-Cluster membership for Area 


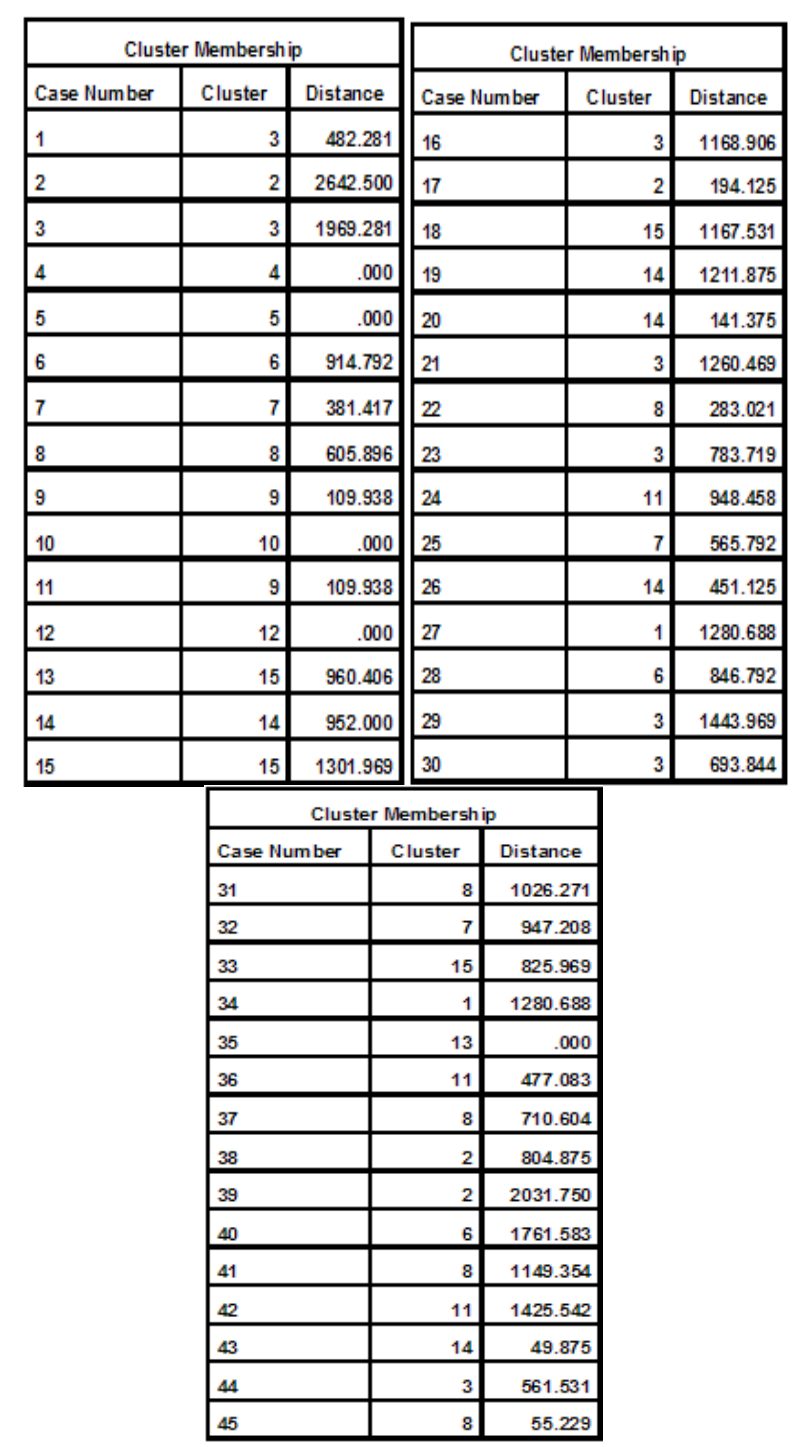

Table 5.6-Cluster membership for Rectangularity

\begin{tabular}{|c|c|c|c|c|c|}
\hline \multicolumn{3}{|c|}{ Cluster Membership } & \multicolumn{3}{|c|}{ Cluster Membership } \\
\hline Case Number & Cluster & Distance & Case Number & Cluster & Distance \\
\hline 1 & 5 & .008 & 16 & 3 & .015 \\
\hline 2 & 2 & .002 & 17 & 3 & .011 \\
\hline 3 & 3 & .004 & 18 & 13 & .014 \\
\hline 4 & 4 & .001 & 19 & 5 & .011 \\
\hline 5 & 4 & .001 & 20 & 5 & .014 \\
\hline 6 & 3 & .005 & 21 & 3 & .012 \\
\hline 7 & 7 & .000 & 22 & 3 & .004 \\
\hline 8 & 1 & .005 & 23 & 5 & .002 \\
\hline 9 & 9 & .004 & 24 & 8 & .006 \\
\hline 10 & 10 & .000 & 25 & 8 & .002 \\
\hline 11 & 11 & .000 & 26 & 6 & .001 \\
\hline 12 & 12 & .000 & 27 & 9 & .004 \\
\hline 13 & 13 & .006 & 28 & 5 & .003 \\
\hline 14 & 14 & .006 & 29 & 6 & .001 \\
\hline 15 & 14 & .001 & 30 & 3 & .013 \\
\hline
\end{tabular}

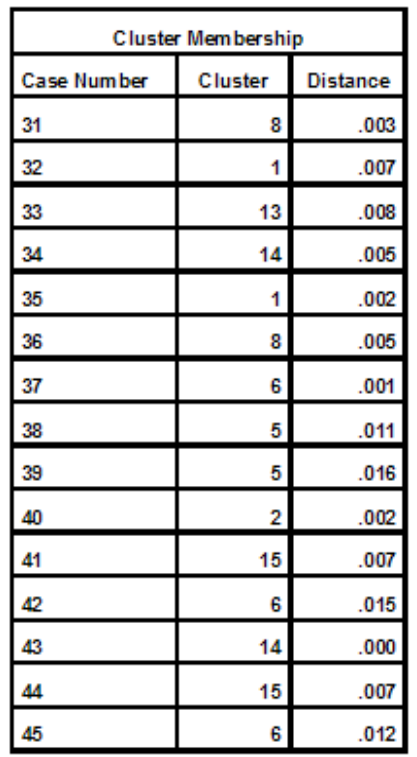

Table 5.7-Cluster membership for Diameter
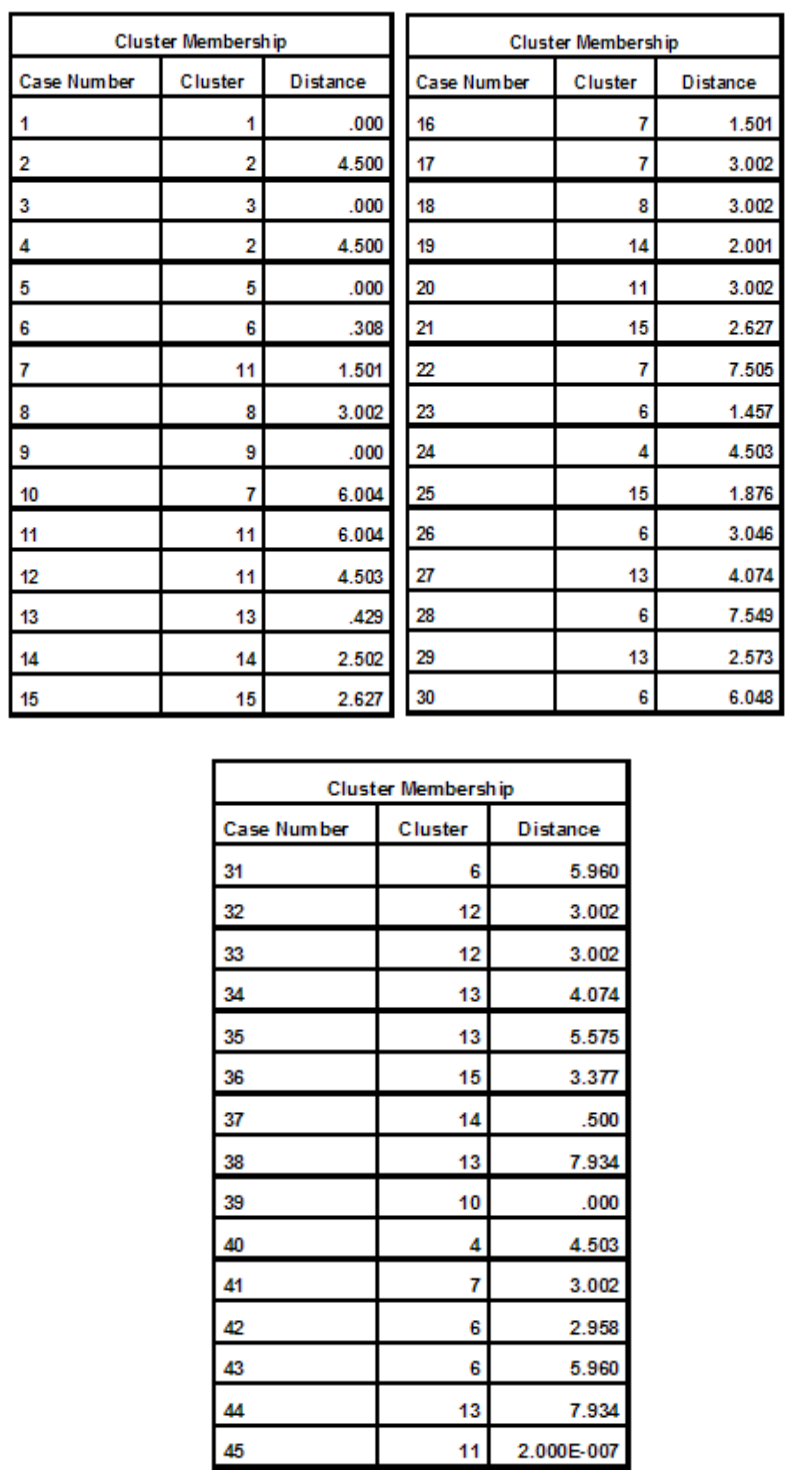
Table 5.8-Cluster membership for Compactness

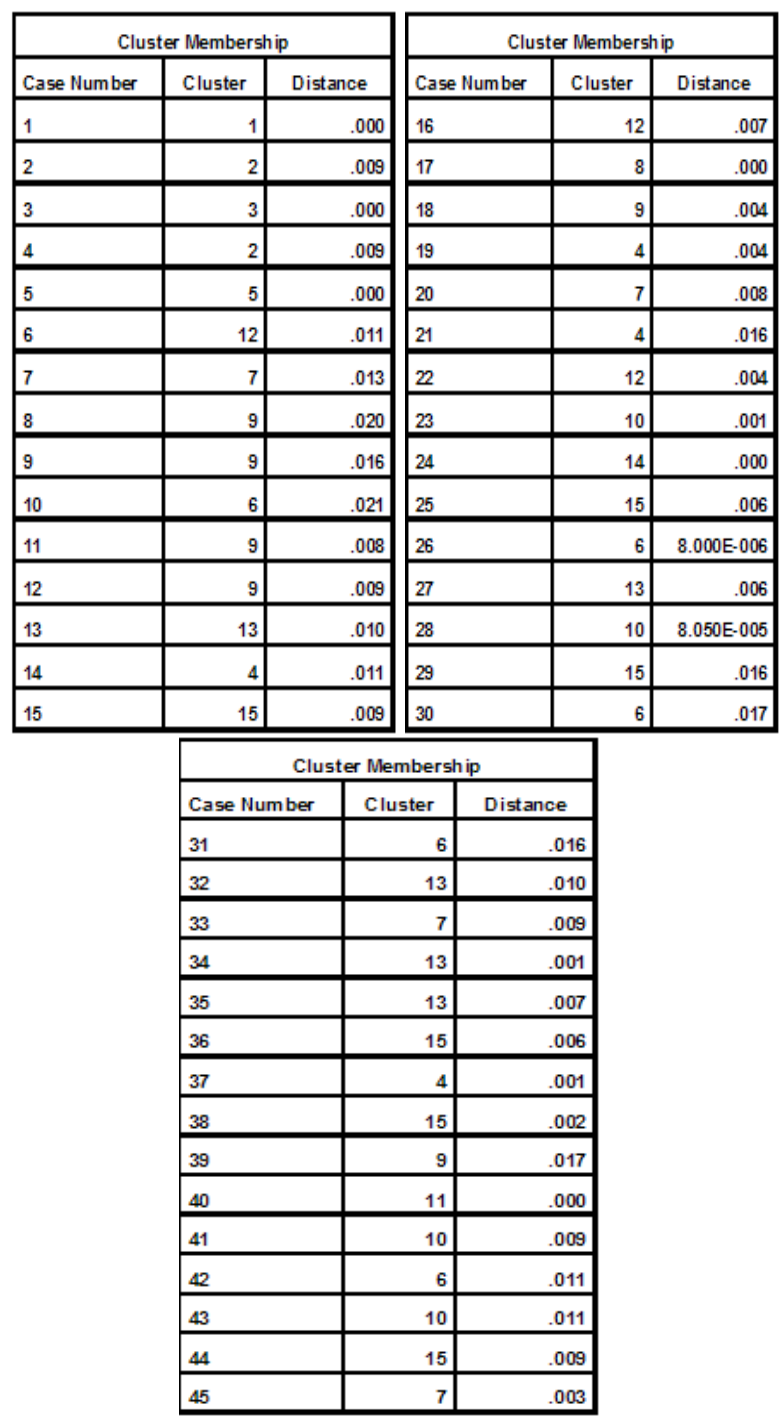

Table 5.9-Cluster membership for Perimeter Ratio of Major Axis-Minor Axis

\begin{tabular}{|c|c|c|c|c|c|}
\hline \multicolumn{3}{|c|}{ Cluster Mem bership } & \multicolumn{3}{|c|}{ Cluster Membership } \\
\hline Case Number & Cluster & Distance & Case Number & Cluster & Distance \\
\hline 1 & 1 & .034 & 16 & 7 & .057 \\
\hline 2 & 2 & .000 & 17 & 14 & .014 \\
\hline 3 & 3 & .000 & 18 & 11 & .023 \\
\hline 4 & 5 & .020 & 19 & 7 & .032 \\
\hline 5 & 5 & .020 & 20 & 9 & .044 \\
\hline 6 & 6 & .025 & 21 & 7 & .062 \\
\hline 7 & 7 & .048 & 22 & 1 & .001 \\
\hline 8 & 8 & .027 & 23 & 14 & .043 \\
\hline 9 & 9 & .015 & 24 & 7 & .039 \\
\hline 10 & 9 & .059 & 25 & 14 & .024 \\
\hline 11 & 11 & .023 & 26 & 13 & .050 \\
\hline 12 & 12 & .000 & 27 & 7 & .063 \\
\hline 13 & 1 & .033 & 28 & 4 & .018 \\
\hline 14 & 14 & .018 & 29 & 13 & .065 \\
\hline 15 & 15 & .030 & 30 & 13 & .012 \\
\hline
\end{tabular}

Cluster Membership

\begin{tabular}{|l|r|r|}
\hline \multicolumn{3}{|c|}{ Cluster Membership } \\
\hline Case Number & Cluster & Distance \\
\hline 31 & 14 & .041 \\
\hline 32 & 13 & .046 \\
\hline 33 & 4 & .018 \\
\hline 34 & 8 & .008 \\
\hline 35 & 14 & .027 \\
\hline 36 & 13 & .047 \\
\hline 37 & 15 & .028 \\
\hline 38 & 8 & .018 \\
\hline 39 & 8 & .046 \\
\hline 40 & 6 & .025 \\
\hline 41 & 15 & .058 \\
\hline 42 & 10 & .000 \\
\hline 43 & 14 & .029 \\
\hline 44 & 13 & .004 \\
\hline 45 & 8 & .007 \\
\hline
\end{tabular}

Table 5.10-Cluster membership for Perimeter Ratio of Diameter
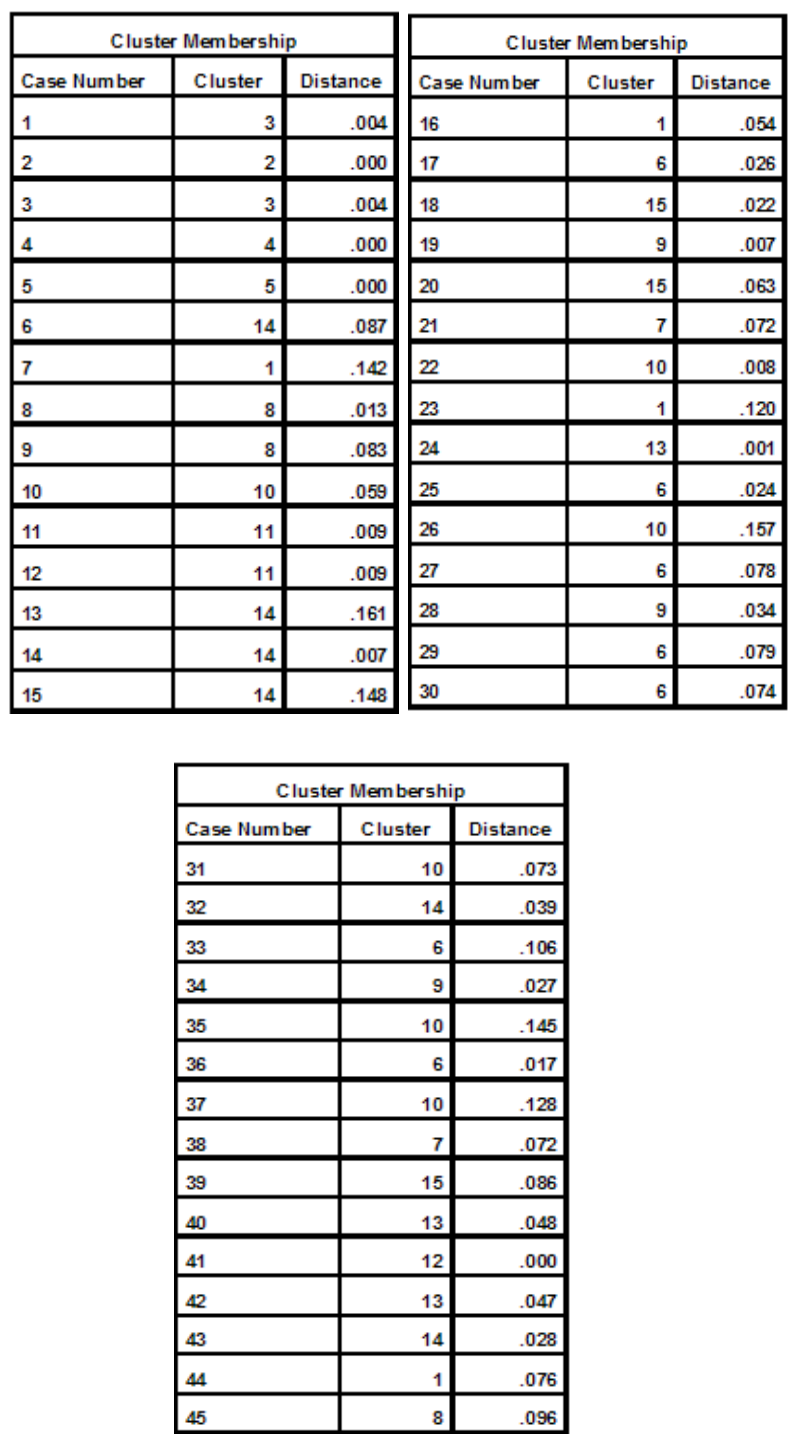

Table 5.11-Cluster membership for Concavity 

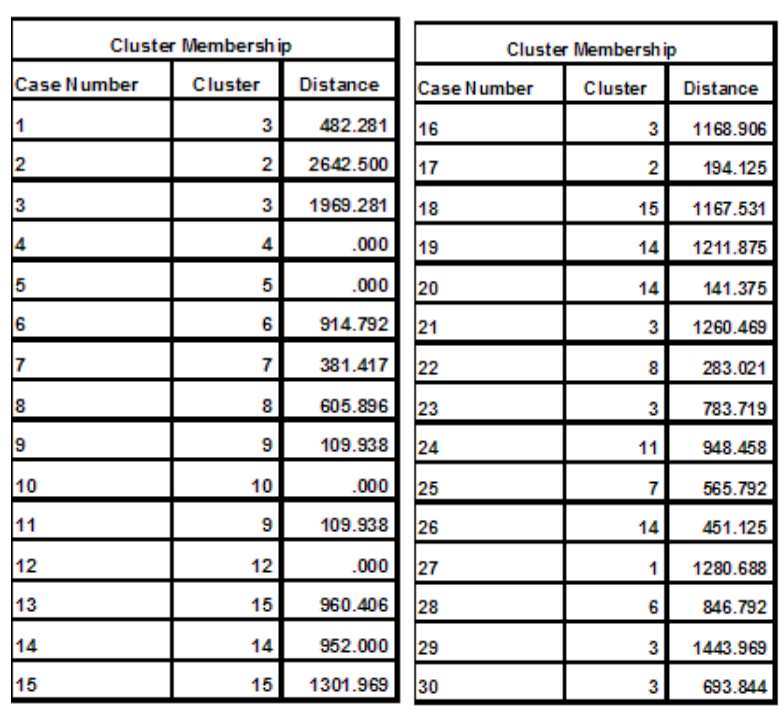

\begin{tabular}{|l|r|r|}
\hline \multicolumn{3}{|c|}{ Cluster Membership } \\
\hline Case Number & Cluster & Distance \\
\hline 31 & 8 & 1026.271 \\
\hline 32 & 7 & 947.208 \\
\hline 33 & 15 & 825.969 \\
\hline 34 & 1 & 1280.688 \\
\hline 35 & 13 & .000 \\
\hline 36 & 11 & 477.083 \\
\hline 37 & 8 & 710.604 \\
\hline 38 & 2 & 804.875 \\
\hline 39 & 2 & 2031.750 \\
\hline 40 & 6 & 1761.583 \\
\hline 41 & 8 & 1149.354 \\
\hline 42 & 11 & 1425.542 \\
\hline 43 & 14 & 49.875 \\
\hline 44 & 3 & 561.531 \\
\hline 45 & 8 & 55.229 \\
\hline
\end{tabular}

Table 5.12-Cluster membership for R-Factor

\begin{tabular}{|l|r|r|}
\hline \multicolumn{3}{|c|}{ Cluster Membership } \\
\hline Case Number & Cluster & Distance \\
\hline 1 & 1 & .000 \\
\hline 2 & 2 & .000 \\
\hline 3 & 3 & .000 \\
\hline 4 & 4 & .000 \\
\hline 5 & 5 & .000 \\
\hline 6 & 6 & 3.420 \\
\hline 7 & 11 & 7.074 \\
\hline 8 & 9 & 1.591 \\
\hline 9 & 9 & 9.163 \\
\hline 10 & 10 & 1.367 \\
\hline 11 & 11 & 12.022 \\
\hline 12 & 11 & .503 \\
\hline 13 & 13 & .602 \\
\hline 14 & 14 & 3.522 \\
\hline 15 & 15 & 3.454 \\
\hline
\end{tabular}

\begin{tabular}{|l|r|r|}
\hline \multicolumn{3}{|c|}{ Cluster Membership } \\
\hline Case Number & Cluster & Distance \\
\hline 16 & 10 & 8.599 \\
\hline 17 & 7 & 3.755 \\
\hline 18 & 9 & 7.571 \\
\hline 19 & 14 & 2.811 \\
\hline 20 & 11 & 2.049 \\
\hline 21 & 15 & 3.454 \\
\hline 22 & 7 & 3.755 \\
\hline 23 & 6 & 5.167 \\
\hline 24 & 12 & 8.423 \\
\hline 25 & 15 & 2.458 \\
\hline 26 & 6 & 1.634 \\
\hline 27 & 13 & 5.083 \\
\hline 28 & 6 & 8.314 \\
\hline 29 & 13 & 3.178 \\
\hline 30 & 6 & 6.101 \\
\hline
\end{tabular}

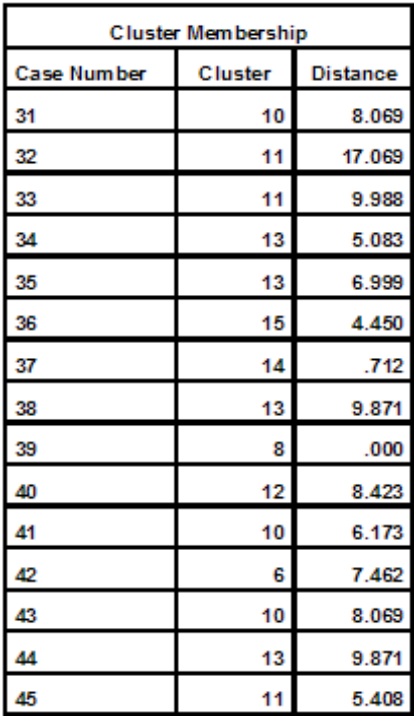

Table 6-Cluster membership of fruit clusters build from 14 individual fruit features

Table 6.1-Cluster membership for Branch Length
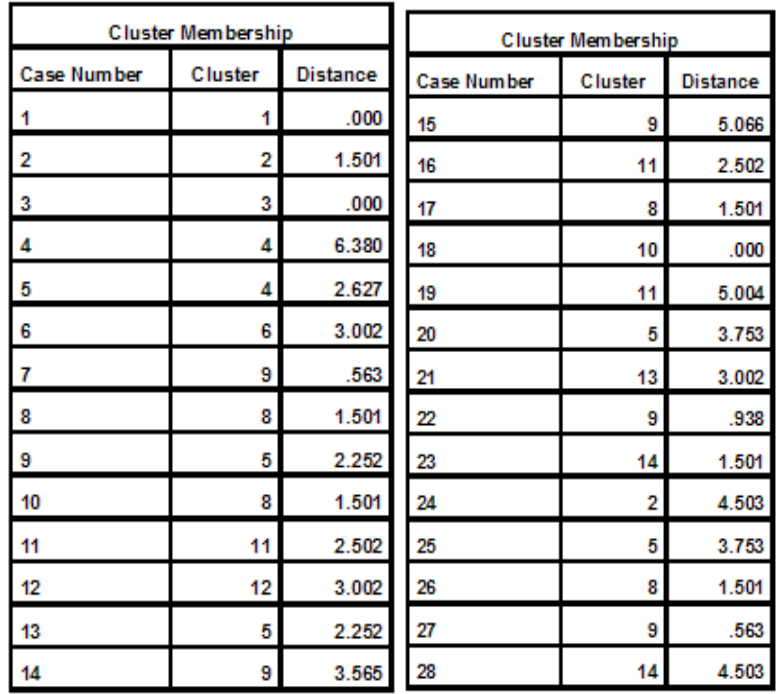

\begin{tabular}{|l|r|r|}
\hline \multicolumn{3}{|c|}{ Cluster Membership } \\
\hline Case Number & Cluster & Distance \\
\hline 29 & 6 & 4.503 \\
\hline 30 & 6 & .000 \\
\hline 31 & 14 & 1.501 \\
\hline 32 & 4 & .375 \\
\hline 33 & 14 & 4.503 \\
\hline 34 & 13 & 3.002 \\
\hline 35 & 9 & 3.940 \\
\hline 36 & 7 & .000 \\
\hline 37 & 2 & 6.004 \\
\hline 38 & 4 & 4.128 \\
\hline 39 & 6 & 1.501 \\
\hline 40 & 9 & 2.439 \\
\hline 41 & 9 & 2.439 \\
\hline 42 & 12 & 3.002 \\
\hline
\end{tabular}


Table 6.2-Cluster membership for Branch Width

\begin{tabular}{|c|c|c|c|c|c|c|}
\hline \multicolumn{3}{|c|}{ Cluster Membership } & \multicolumn{4}{|c|}{ Cluster Membership } \\
\hline Case Number & Cluster & Distance & & Number & Cluster & Distance \\
\hline 1 & 8 & 5.629 & 15 & & 2 & .500 \\
\hline 2 & 2 & 4.003 & 16 & & 7 & 4.803 \\
\hline 3 & 3 & 3.753 & 17 & & 7 & 7.205 \\
\hline 4 & 4 & 4.503 & 18 & & 1 & 5.254 \\
\hline 5 & 5 & 3.377 & 19 & & 1 & 5.254 \\
\hline 6 & 14 & 4.503 & 20 & & 12 & .375 \\
\hline 7 & 8 & 2.627 & 21 & & 5 & 7.881 \\
\hline 8 & 8 & .375 & 22 & & 3 & 3.753 \\
\hline 9 & g & 1.501 & 23 & & 8 & 7.881 \\
\hline 10 & 7 & .300 & 24 & & 9 & .000 \\
\hline 11 & 11 & 1.201 & 25 & & 5 & 5.629 \\
\hline 12 & 9 & 4.503 & 26 & & 11 & .300 \\
\hline 13 & 2 & 3.502 & 27 & & 12 & 4.128 \\
\hline 14 & 14 & 1.501 & 28 & & 12 & .375 \\
\hline & & uster Mem & grsh & & & \\
\hline & Case Numbe & $\mathrm{Clu}$ & & Distance & & \\
\hline & 29 & & 12 & 3.377 & & \\
\hline & 30 & & 9 & 3.002 & & \\
\hline & 31 & & 7 & 1.801 & & \\
\hline & 32 & & 11 & 4.803 & & \\
\hline & 33 & & 11 & .300 & & \\
\hline & 34 & & 11 & 4.203 & & \\
\hline & 35 & & 5 & 5.629 & & \\
\hline & 36 & & 7 & 300 & & \\
\hline & 37 & & 10 & .000 & & \\
\hline & 38 & & 13 & .000 & & \\
\hline & 39 & & 6 & .000 & & \\
\hline & 40 & & 4 & 1.501 & & \\
\hline & 41 & & 4 & 3.002 & & \\
\hline & 42 & & 14 & 3.002 & & \\
\hline
\end{tabular}

Table 6.3-Cluster membership for Length Width Ratio

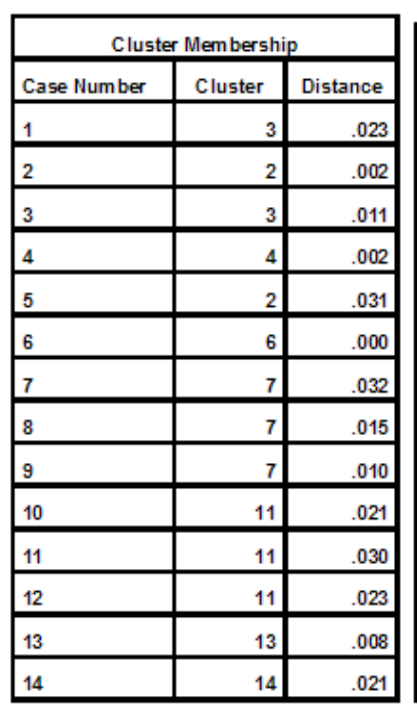

\begin{tabular}{|l|r|r|}
\hline \multicolumn{3}{|c|}{ Cluster Membership } \\
\hline Case Number & Cluster & Distance \\
\hline 15 & 12 & .027 \\
\hline 16 & 7 & .034 \\
\hline 17 & 11 & .002 \\
\hline 18 & 11 & .028 \\
\hline 19 & 8 & .009 \\
\hline 20 & 12 & .004 \\
\hline 21 & 8 & .015 \\
\hline 22 & 7 & .011 \\
\hline 23 & 11 & .016 \\
\hline 24 & 8 & .018 \\
\hline 25 & 5 & .015 \\
\hline 26 & 8 & .023 \\
\hline 27 & 12 & .031 \\
\hline 28 & 14 & .021 \\
\hline
\end{tabular}

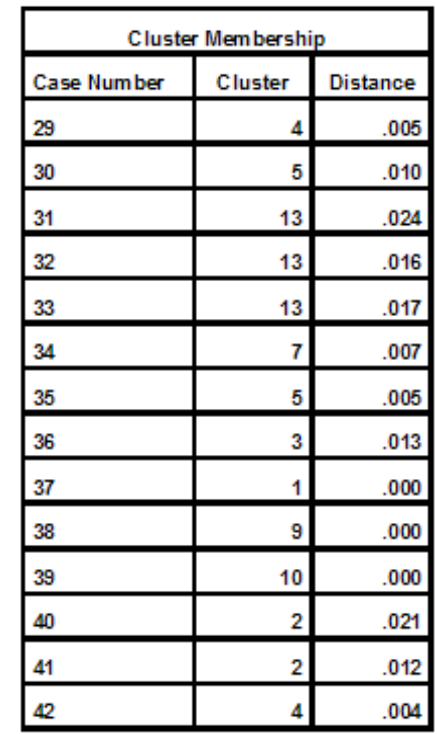

Table 6.4-Cluster membership for Area
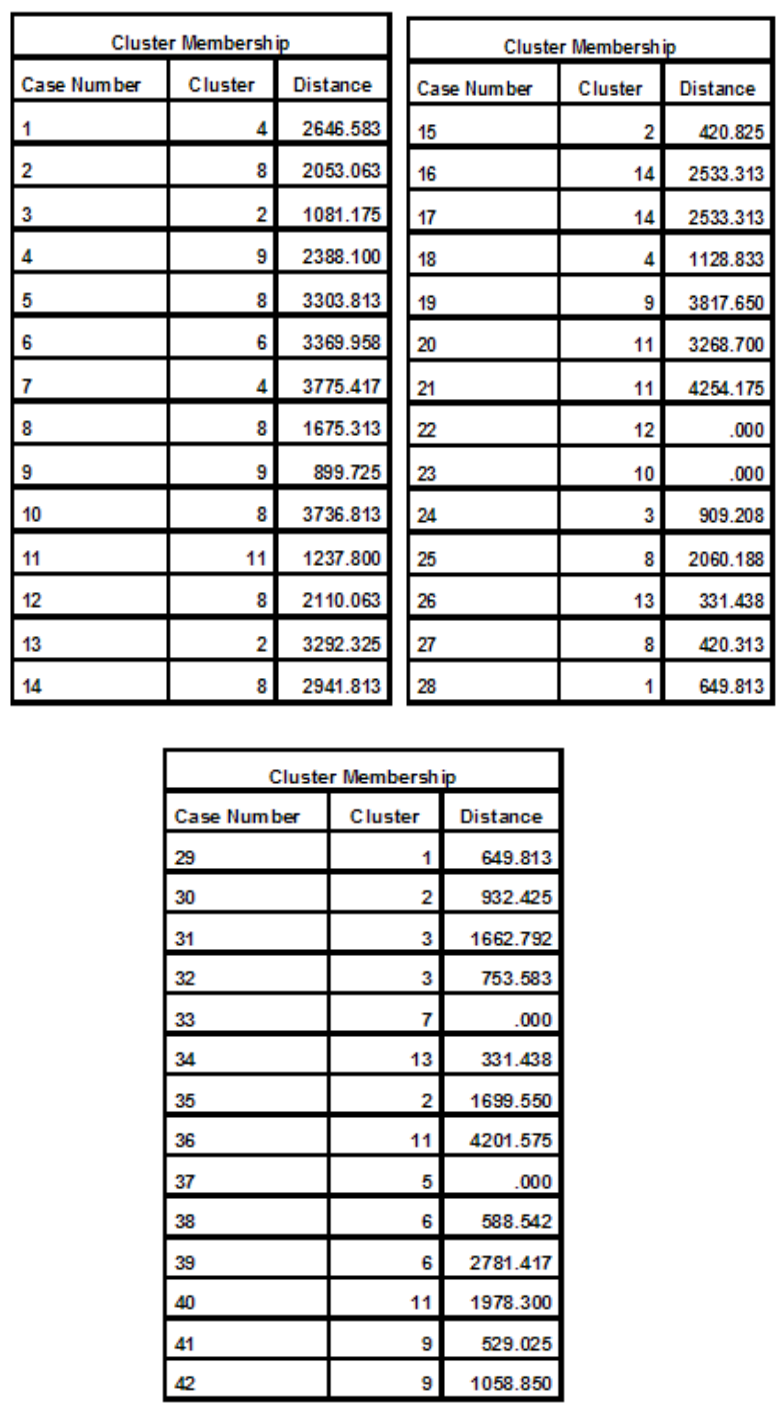

Table 6.5-Cluster membership for Perimeter 

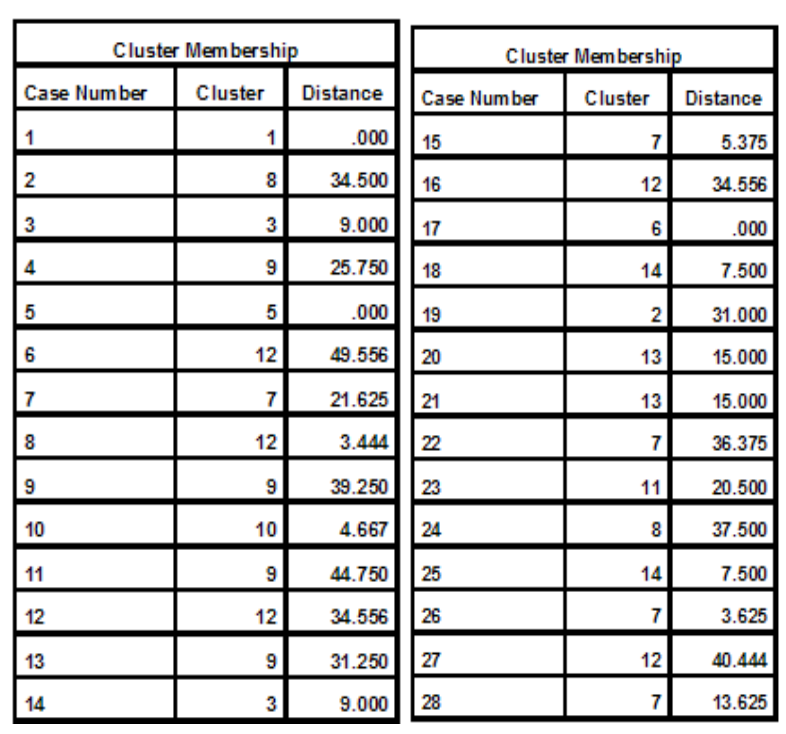

\begin{tabular}{|l|r|r|}
\hline \multicolumn{3}{|c|}{ Cluster Membership } \\
\hline Case Number & Cluster & Distance \\
\hline 29 & 7 & 20.625 \\
\hline 30 & 10 & 21.667 \\
\hline 31 & 12 & 2.444 \\
\hline 32 & 12 & 39.444 \\
\hline 33 & 7 & 11.375 \\
\hline 34 & 11 & 20.500 \\
\hline 35 & 4 & .000 \\
\hline 36 & 12 & 10.444 \\
\hline 37 & 7 & 6.375 \\
\hline 38 & 10 & 26.333 \\
\hline 39 & 12 & 22.444 \\
\hline 40 & 8 & 30.500 \\
\hline 41 & 8 & 33.500 \\
\hline 42 & 2 & 31.000 \\
\hline
\end{tabular}

Table 6.6-Cluster membership for Equivalent Diameter

\begin{tabular}{|c|c|c|c|c|c|}
\hline \multicolumn{3}{|c|}{ Cluster Membership } & \multicolumn{3}{|c|}{ Cluster Membership } \\
\hline Case Number & Cluster & Distance & Case Number & Cluster & Distance \\
\hline 1 & 4 & 3.052 & 15 & 2 & .516 \\
\hline 2 & 8 & 2.413 & 16 & 14 & 3.078 \\
\hline 3 & 2 & 1.340 & 17 & 14 & 3.078 \\
\hline 4 & 9 & 2.705 & 18 & 4 & 1.309 \\
\hline 5 & 8 & 3.882 & 19 & 9 & 4.311 \\
\hline 6 & 6 & 3.687 & 20 & 11 & 3.633 \\
\hline 7 & 4 & 4.361 & 21 & 11 & 4.737 \\
\hline 8 & 8 & 1.967 & 22 & 12 & .000 \\
\hline 9 & 9 & 1.015 & 23 & 10 & .000 \\
\hline 10 & 8 & 4.387 & 24 & 3 & 1.183 \\
\hline 11 & 11 & 1.366 & 25 & 8 & 2.422 \\
\hline 12 & 8 & 2.485 & 26 & 13 & .466 \\
\hline 13 & 2 & 4.083 & 27 & 8 & .487 \\
\hline 14 & 8 & 3.465 & 28 & 1 & .87 \\
\hline
\end{tabular}

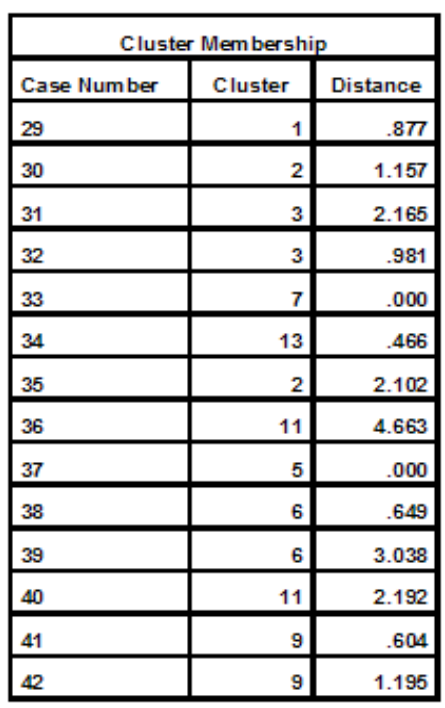

Table 6.7-Cluster membership for Rectangularity
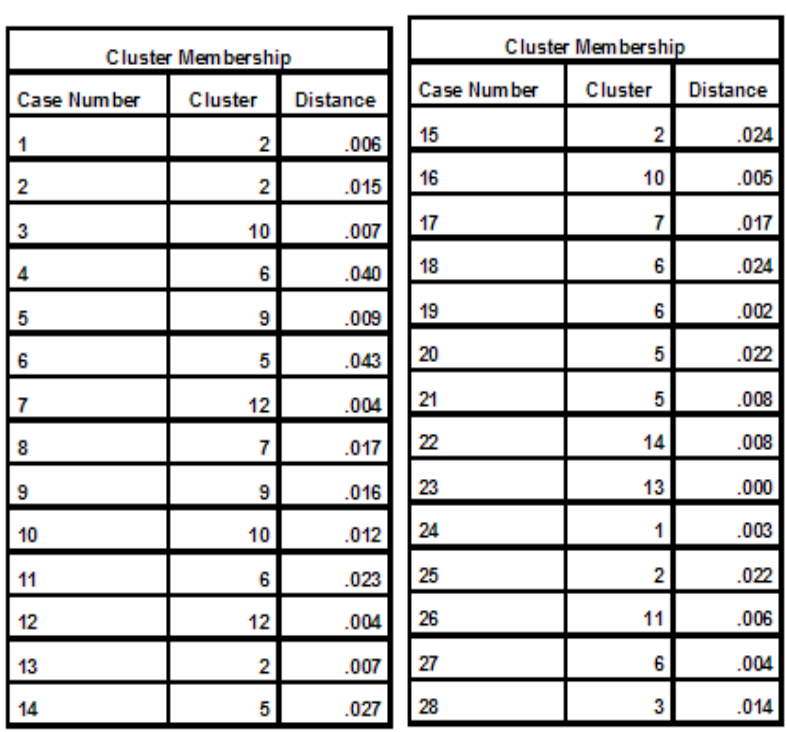


\begin{tabular}{|l|r|r|}
\hline \multicolumn{3}{|c|}{ Cluster Membership } \\
\hline Case Number & Cluster & Distance \\
\hline 29 & 3 & .014 \\
\hline 30 & 1 & .003 \\
\hline 31 & 11 & .006 \\
\hline 32 & 8 & .000 \\
\hline 33 & 14 & .001 \\
\hline 34 & 14 & .009 \\
\hline 35 & 9 & .007 \\
\hline 36 & 5 & .018 \\
\hline 37 & 4 & .013 \\
\hline 38 & 4 & .013 \\
\hline 39 & 5 & .016 \\
\hline 40 & 5 & .022 \\
\hline 41 & 5 & .002 \\
\hline 42 & 5 & .003 \\
\hline
\end{tabular}

Table 6.8-Cluster membership for Diameter

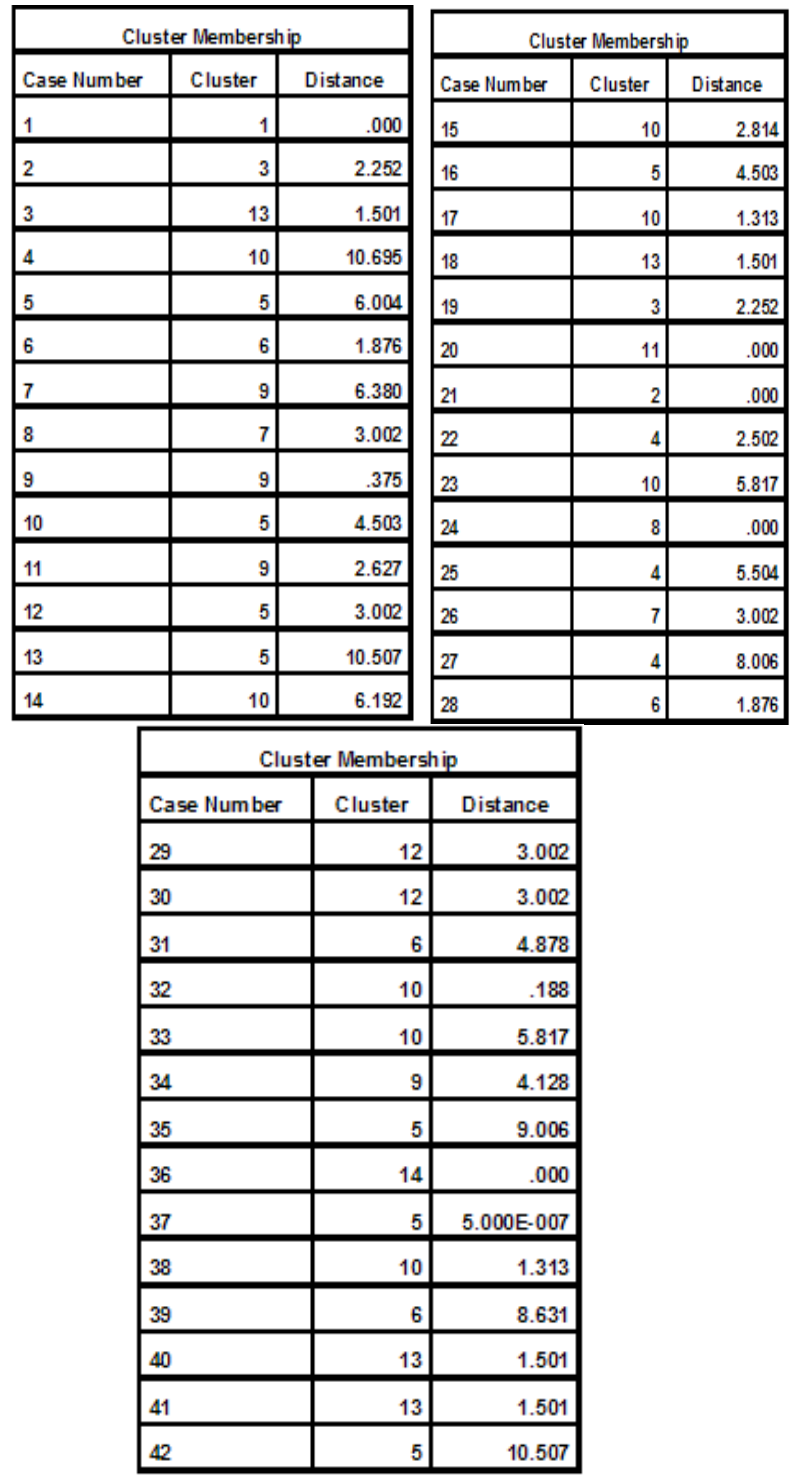

Table 6.9-Cluster membership for Perimeter Ratio of Branch Length-Branch Width
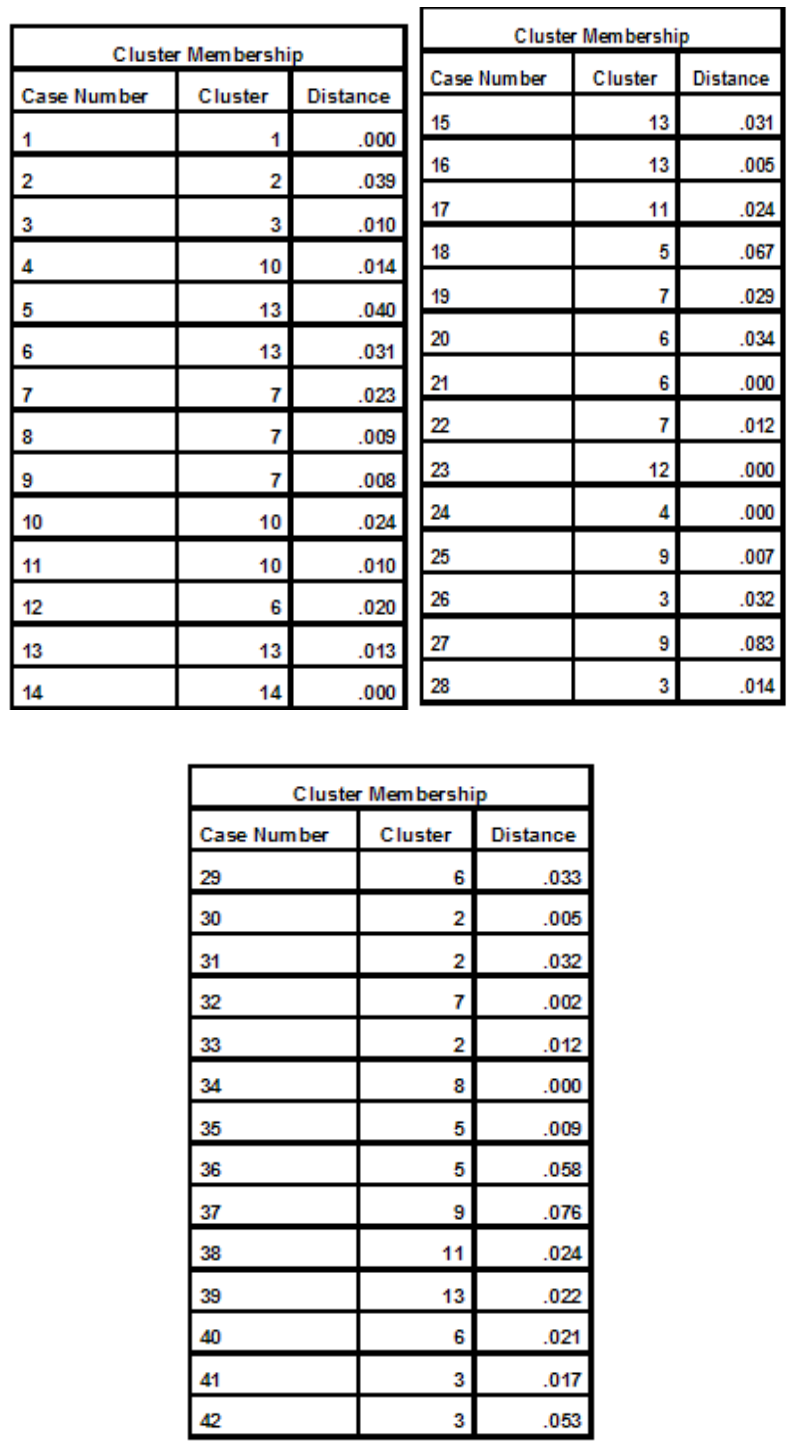

Table 6.10-Cluster membership for Perimeter Ratio of Diameter
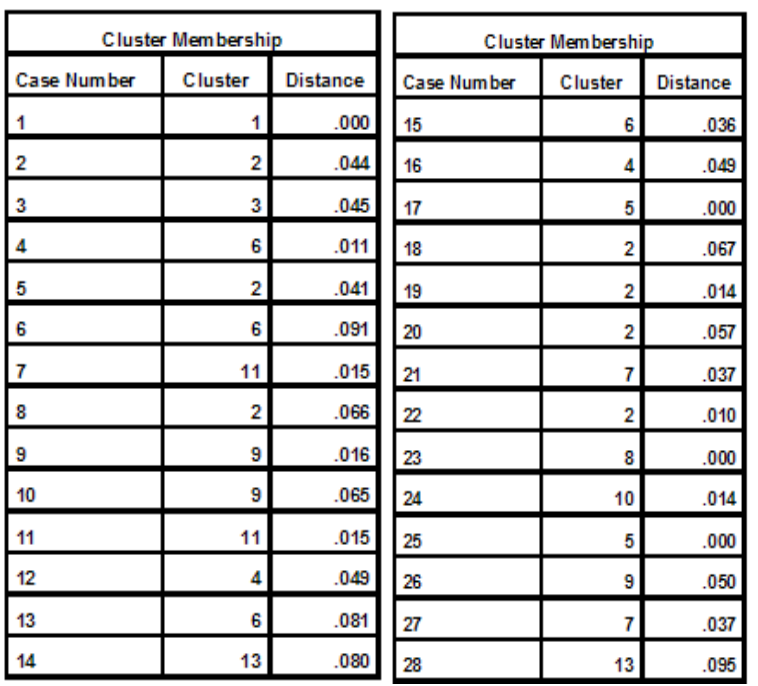


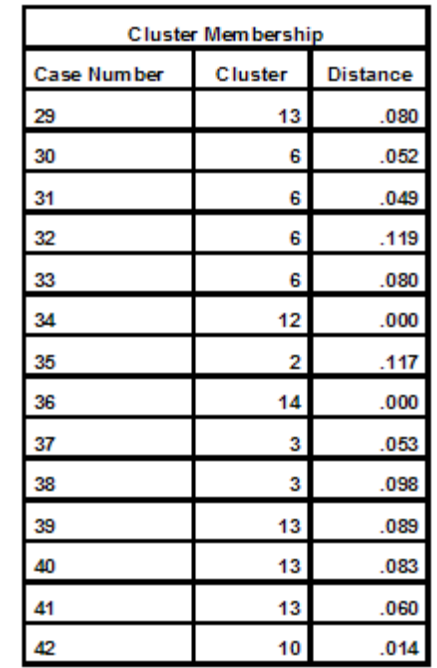

Table 6.11-Cluster membership for Convexity
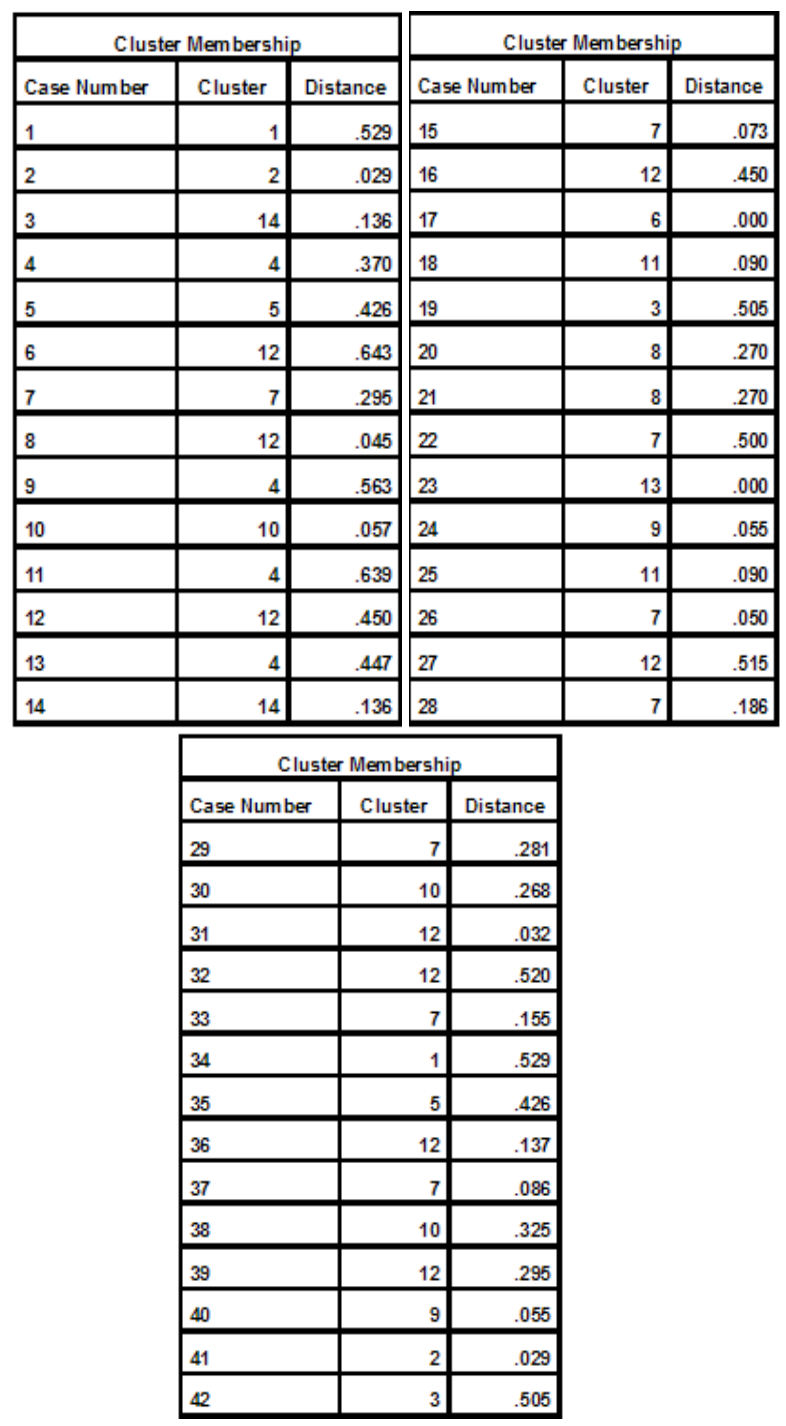

Table 6.12-Cluster membership for Solidity

\begin{tabular}{|l|r|r||l|r|r|}
\hline \multicolumn{3}{|c|}{ Cluster Membership } & \multicolumn{3}{c|}{ Cluster Membership } \\
\hline Case Number & Cluster & Distance & Case Number & Cluster & Distance \\
\hline 1 & 4 & .007 & 15 & 2 & .001 \\
\hline 2 & 8 & .005 & 16 & 14 & .007 \\
\hline 3 & 2 & .003 & 17 & 14 & .007 \\
\hline 4 & 9 & .006 & 18 & 4 & .003 \\
\hline 5 & 8 & .009 & 19 & 9 & .010 \\
\hline 6 & 6 & .009 & 20 & 11 & .009 \\
\hline 7 & 4 & .010 & 21 & 11 & .011 \\
\hline 8 & 8 & .005 & 22 & 12 & .000 \\
\hline 9 & 9 & .002 & 23 & 10 & .000 \\
\hline 10 & 8 & .010 & 24 & 3 & .003 \\
\hline 11 & 11 & .003 & 25 & 8 & .006 \\
\hline 12 & 8 & .006 & 26 & 13 & .000 \\
\hline 13 & 2 & .009 & 27 & 8 & .001 \\
\hline 14 & 8 & .008 & 28 & 1 & .002 \\
\hline
\end{tabular}

\begin{tabular}{|l|r|r|}
\hline \multicolumn{3}{|c|}{ Cluster Membership } \\
\hline Case Number & Cluster & Distance \\
\hline 29 & 1 & .002 \\
\hline 30 & 2 & .002 \\
\hline 31 & 3 & .004 \\
\hline 32 & 3 & .002 \\
\hline 33 & 7 & .000 \\
\hline 34 & 3 & .001 \\
\hline 35 & 2 & .005 \\
\hline 36 & 11 & .011 \\
\hline 37 & 5 & .000 \\
\hline 38 & 6 & .002 \\
\hline 39 & 6 & .007 \\
\hline 40 & 11 & .005 \\
\hline 41 & 9 & .001 \\
\hline 42 & 9 & .003 \\
\hline
\end{tabular}

Table 6.13-Cluster membership for On Pixels

\begin{tabular}{|l|r|r|}
\hline \multicolumn{3}{|c|}{ Cluster Membership } \\
\hline Case Number & Cluster & Distance \\
\hline 1 & 4 & 2664.667 \\
\hline 2 & 8 & 2026.250 \\
\hline 3 & 2 & 1087.200 \\
\hline 4 & 9 & 2399.800 \\
\hline 5 & 8 & 3284.750 \\
\hline 6 & 6 & 3376.333 \\
\hline 7 & 4 & 3777.333 \\
\hline 8 & 8 & 1670.250 \\
\hline 9 & 9 & 902.800 \\
\hline 10 & 8 & 3740.750 \\
\hline 11 & 11 & 1240.000 \\
\hline 12 & 8 & 2109.750 \\
\hline 13 & 2 & 3286.800 \\
\hline 14 & 8 & 2930.250 \\
\hline
\end{tabular}

\begin{tabular}{|l|r|r|}
\hline \multicolumn{3}{|c|}{ Cluster Membership } \\
\hline Case Number & Cluster & Distance \\
\hline 15 & 2 & 418.800 \\
\hline 16 & 14 & 2549.500 \\
\hline 17 & 14 & 2549.500 \\
\hline 18 & 4 & 1112.667 \\
\hline 19 & 9 & 3831.200 \\
\hline 20 & 11 & 3284.000 \\
\hline 21 & 11 & 4251.000 \\
\hline 22 & 12 & .000 \\
\hline 23 & 10 & .000 \\
\hline 24 & 3 & 927.333 \\
\hline 25 & 8 & 2075.250 \\
\hline 26 & 13 & 316.000 \\
\hline 27 & 8 & 433.250 \\
\hline 28 & 1 & 649.500 \\
\hline
\end{tabular}




\begin{tabular}{|l|r|r|}
\hline \multicolumn{3}{|c|}{ Cluster Membership } \\
\hline Case Number & Cluster & Distance \\
\hline 29 & 1 & 649.500 \\
\hline 30 & 2 & 931.200 \\
\hline 31 & 3 & 1671.667 \\
\hline 32 & 3 & 744.333 \\
\hline 33 & 7 & .000 \\
\hline 34 & 13 & 316.000 \\
\hline 35 & 2 & 1687.200 \\
\hline 36 & 11 & 4187.000 \\
\hline 37 & 5 & .000 \\
\hline 38 & 6 & 585.667 \\
\hline 39 & 6 & 2790.667 \\
\hline 40 & 11 & 1980.000 \\
\hline 41 & 9 & 530.200 \\
\hline 42 & 9 & 1058.800 \\
\hline
\end{tabular}

Table 6.14-Cluster membership for Narrow-Factor

\begin{tabular}{|l|r|r|}
\hline \multicolumn{3}{|c|}{ Cluster Membership } \\
\hline Case Number & Cluster & Distance \\
\hline 1 & 2 & .005 \\
\hline 2 & 2 & .005 \\
\hline 3 & 3 & .000 \\
\hline 4 & 7 & .003 \\
\hline 5 & 5 & .000 \\
\hline 6 & 6 & .000 \\
\hline 7 & 7 & .001 \\
\hline 8 & 6 & .001 \\
\hline 9 & 9 & .001 \\
\hline 10 & 10 & .000 \\
\hline 11 & 11 & .000 \\
\hline 12 & 11 & .001 \\
\hline 13 & 8 & .002 \\
\hline 14 & 14 & .005 \\
\hline
\end{tabular}

\begin{tabular}{|l|r|r|}
\hline \multicolumn{3}{|c|}{ Cluster Membership } \\
\hline Case Number & Cluster & Distance \\
\hline 15 & 14 & .008 \\
\hline 16 & 8 & .005 \\
\hline 17 & 14 & .003 \\
\hline 18 & 8 & .007 \\
\hline 19 & 4 & .001 \\
\hline 20 & 1 & .000 \\
\hline 21 & 4 & .001 \\
\hline 22 & 12 & .000 \\
\hline 23 & 7 & .003 \\
\hline 24 & 6 & .001 \\
\hline 25 & 7 & .001 \\
\hline 26 & 7 & .001 \\
\hline 27 & 5 & .000 \\
\hline 28 & 7 & .001 \\
\hline
\end{tabular}

\begin{tabular}{|l|r|r|}
\hline \multicolumn{3}{|c|}{ Cluster Membership } \\
\hline Case Number & Cluster & Distance \\
\hline 29 & 7 & .001 \\
\hline 30 & 9 & .001 \\
\hline 31 & 7 & .001 \\
\hline 32 & 7 & .004 \\
\hline 33 & 7 & .001 \\
\hline 34 & 13 & .006 \\
\hline 35 & 13 & .006 \\
\hline 36 & 7 & .001 \\
\hline 37 & 7 & .001 \\
\hline 38 & 7 & .001 \\
\hline 39 & 7 & .001 \\
\hline 40 & 11 & .001 \\
\hline 41 & 11 & .005 \\
\hline 42 & 11 & .003 \\
\hline
\end{tabular}

After we obtain the clusters, it is to be judged that which feature bears the best cluster formation capability. Feature having the best cluster formation capability is the most important feature for the cluster analysis. K-Means clustering algorithm does not give this feature importance measure. Hence we discuss how this measure is obtained and compare in our experiment -

We have used total 15 different classes of tomato leaves and 14 classes of tomato fruits, with each class consisting of 3 cases (patterns). Now it is to be seen that how many cases of a particular class are included in a same cluster. More is the number of cases of a particular class included in same cluster, better is clustering result. We calculate a measure called "Same Cluster Membership Ratio (SCMR)" for each class from the cluster membership (Table 5 and Table 6) produced from a particular feature and then finding the summation of SCMRs (Total SCMR) of all classes. Higher is the value of Total SCMR of a feature, higher is its importance in cluster formation. SCMR for a particular class can be calculated by finding the ratio of the total number of cases of that class included in same cluster and total number of cases present in that class (in our experiment, this value is 3 for all classes) .

Now from the above definition of SCMR, it is obvious that in our problem, we will obtain one of the following three SCMR values for each class under the following conditions-

i) If no case belonging to a particular class is included in same cluster, then SCMR of that class is 0 .

ii) If 2 cases of a class are included in same cluster, then SCMR of that class is $2 / 3=0.67$ (approx).

iii) If all 3 cases of a class are included in same cluster, then SCMR of that class is $3 / 3=1$.

Thus, the range of values of SCMR in our problem is 0 to 1 .

Table 7 and Table 8 show the class number and the corresponding three case numbers (pattern number) that belong to that particular class. Cluster number is the number of the cluster; the case pattern is a member of. And the last field of Table 7 and table 8 is the calculated SCMR value of each class for each feature variables of tomato leaf and fruit. The last row of each table shows the Total SCMR value related to each leaf/fruit feature---

Table 7-SCMR calculation for individual leaf features 
International Journal of Computer Applications Technology and Research Volume 2- Issue 5, 557 - 586, 2013, ISSN: 2319-8656

SCMR calculation for Major Axis

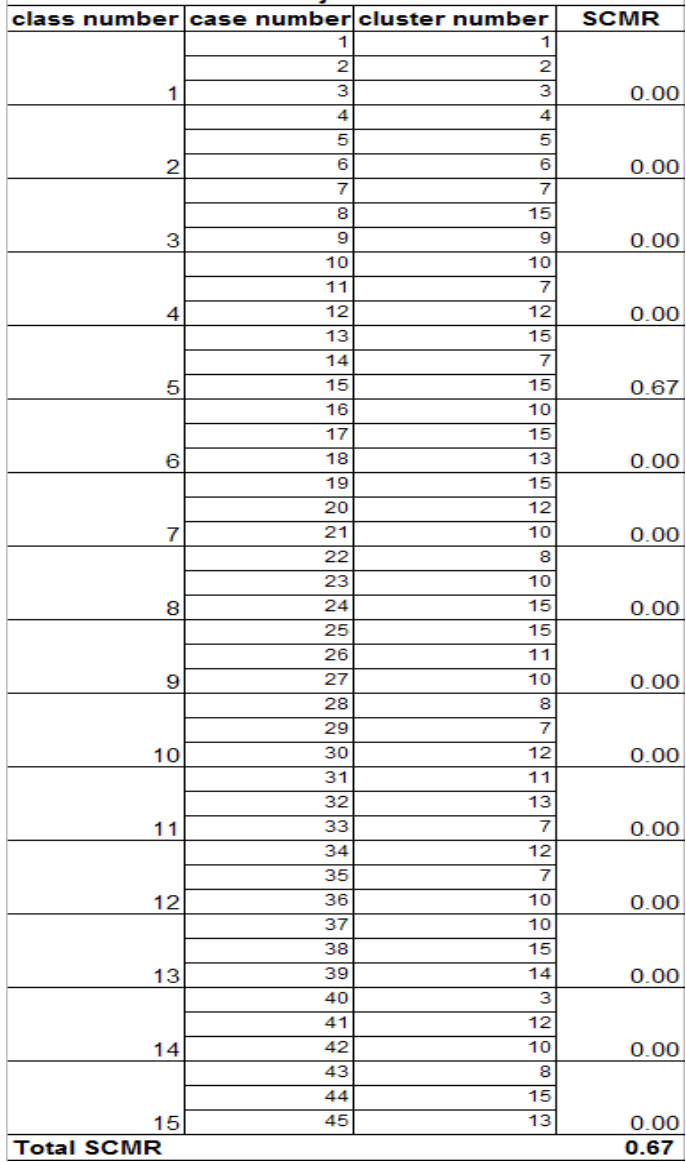

SCMR calculation for Minor Axis \begin{tabular}{|l|l|l|l|}
\hline class number & case number & cluster number & SCMR \\
\hline
\end{tabular}

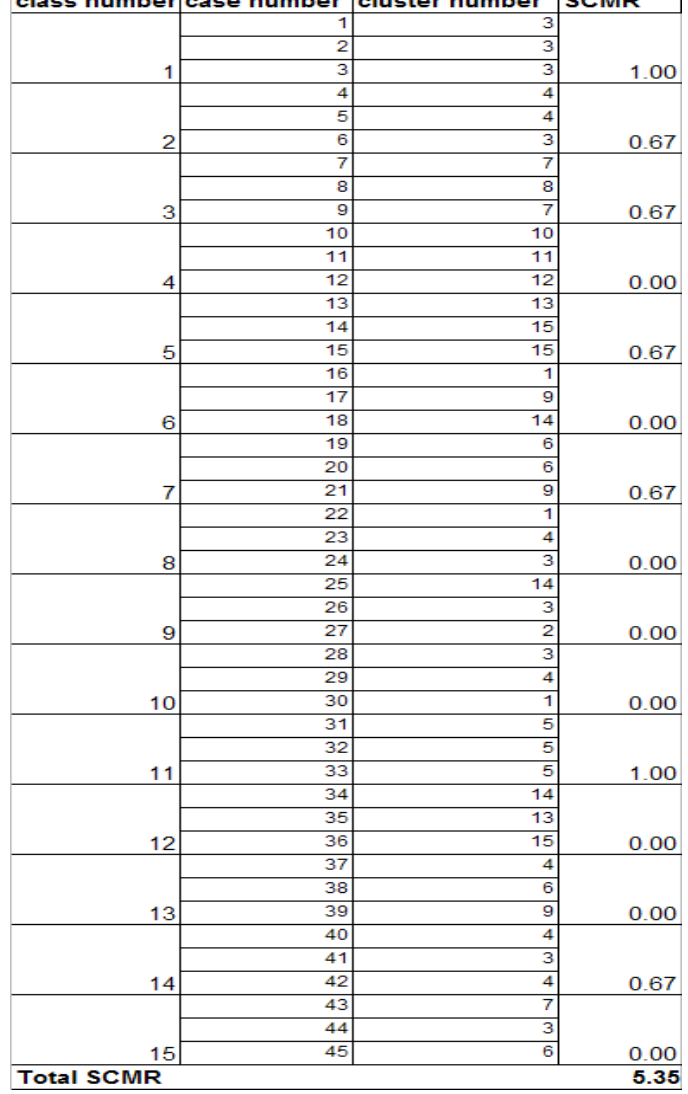

SCMR calculation for Aspect Ratio

class number case number cluster number SCMR

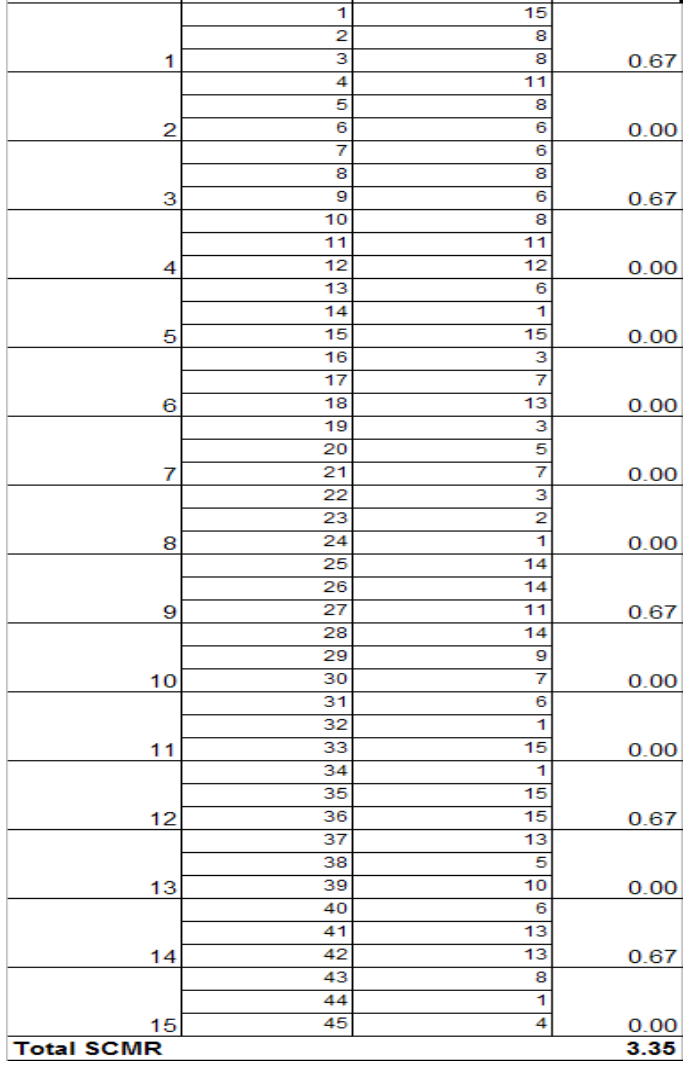

SCMR calculation for Eccentricity

class number case number|cluster number SCMR

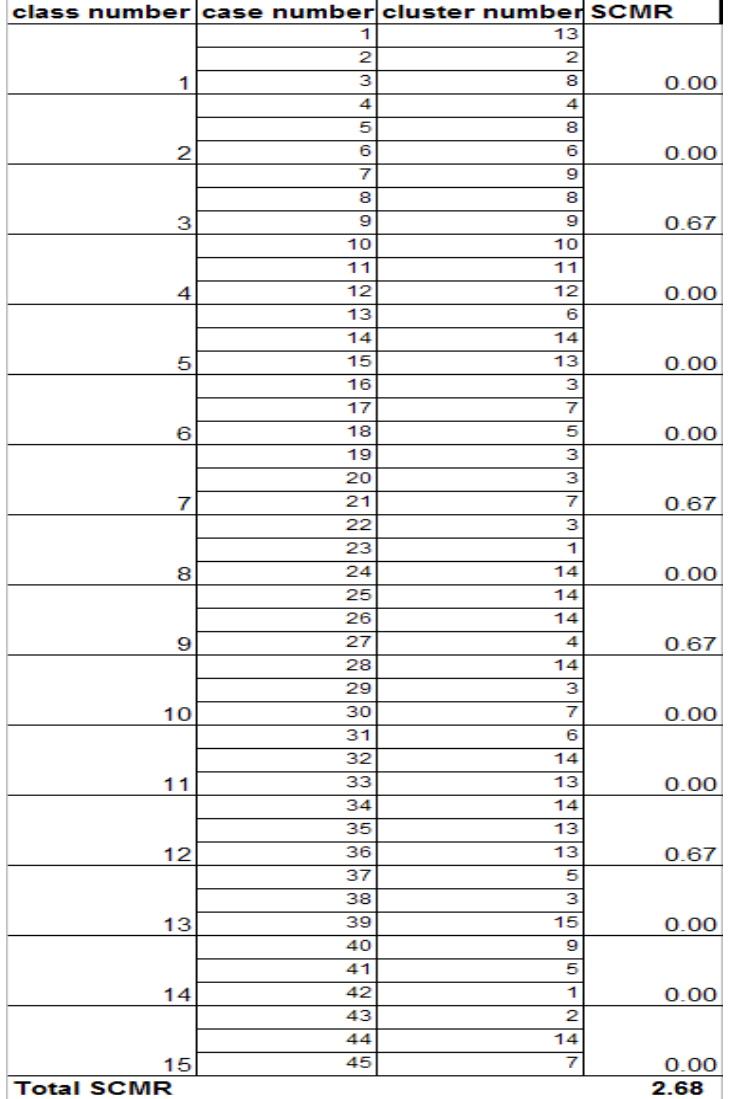


International Journal of Computer Applications Technology and Research Volume 2- Issue 5, 557 - 586, 2013, ISSN: 2319-8656

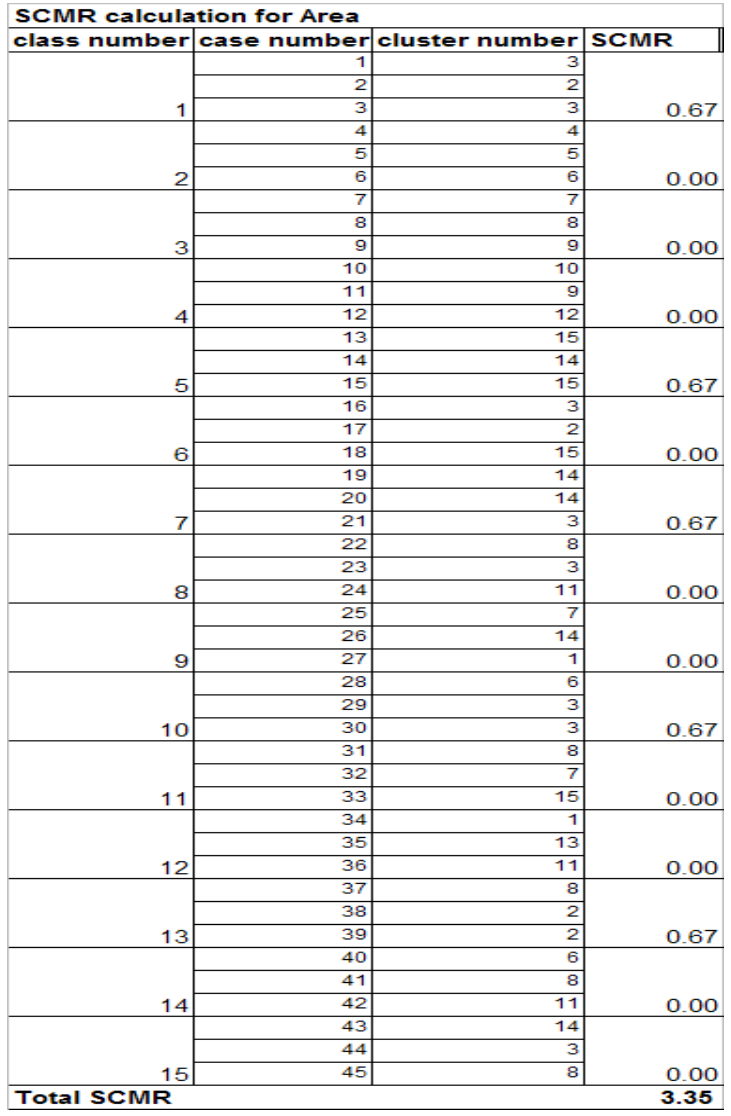

SCMR calculation for Rectangularity

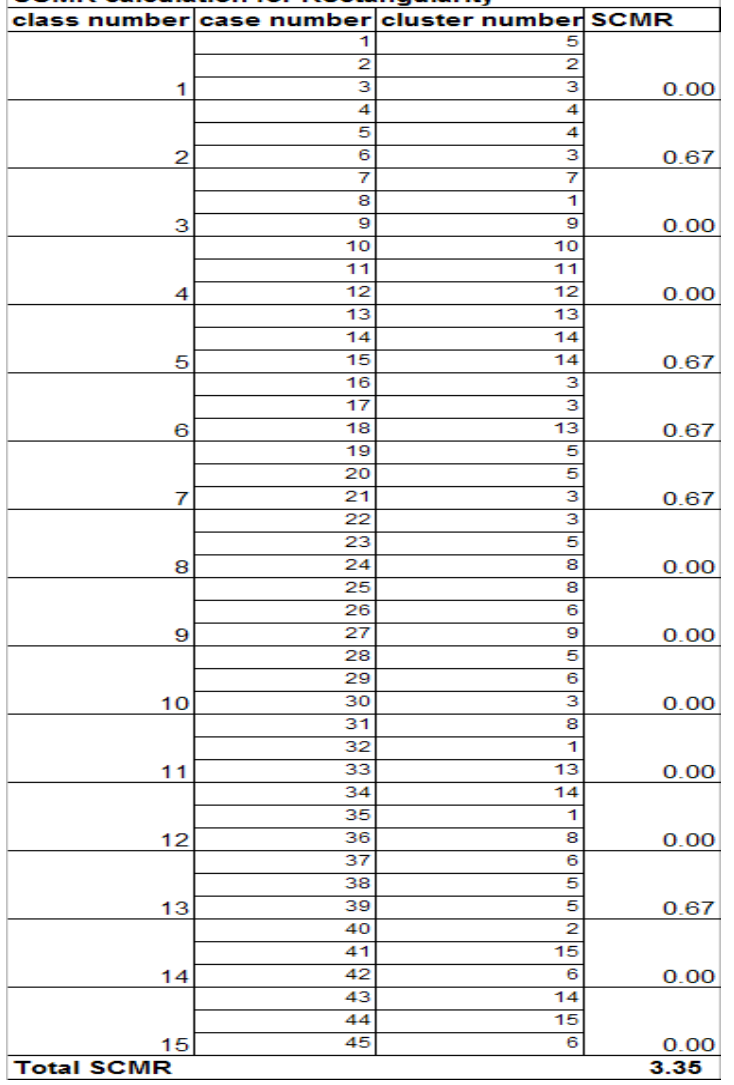

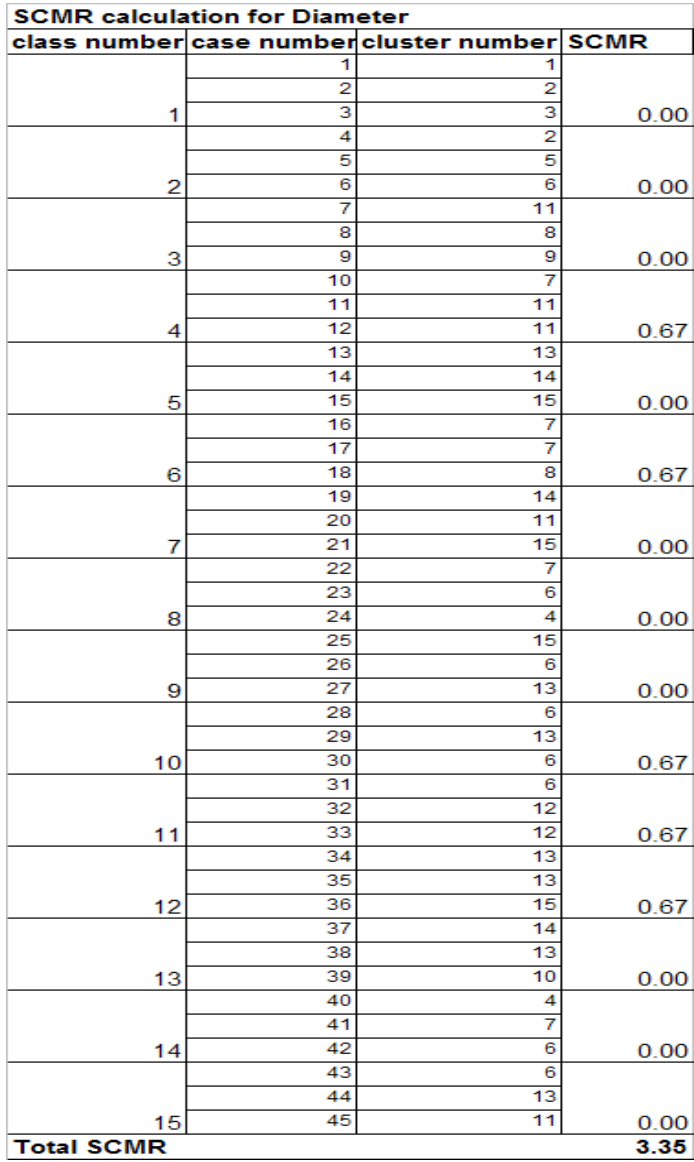

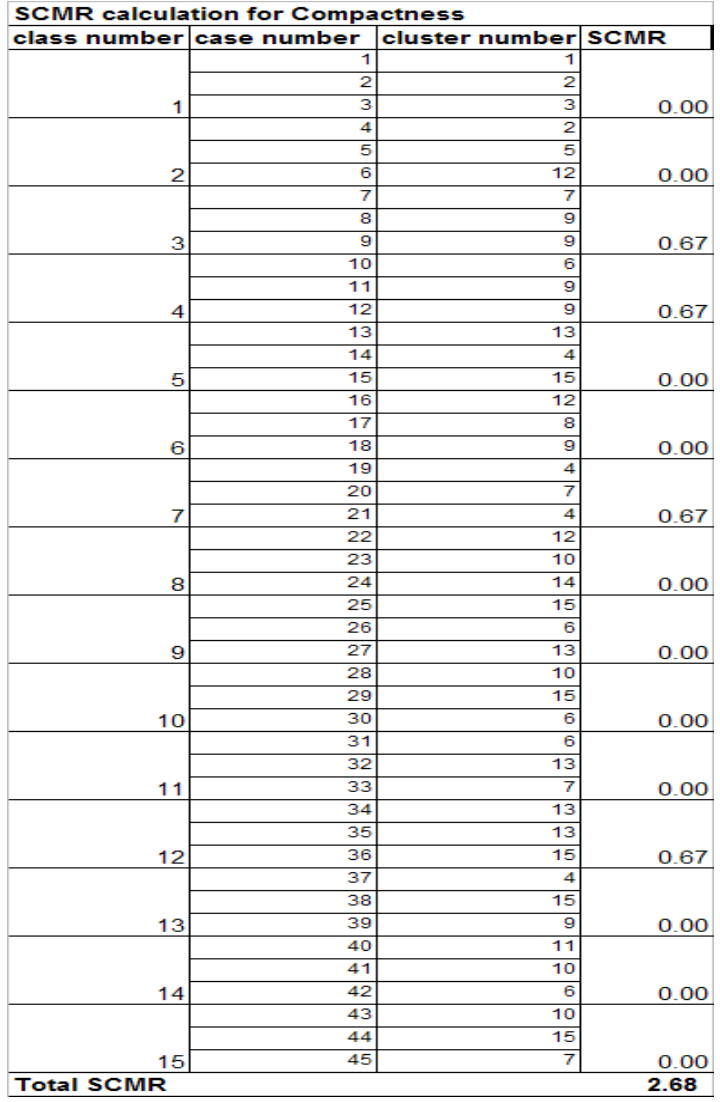


International Journal of Computer Applications Technology and Research Volume 2- Issue 5, 557 - 586, 2013, ISSN: 2319-8656
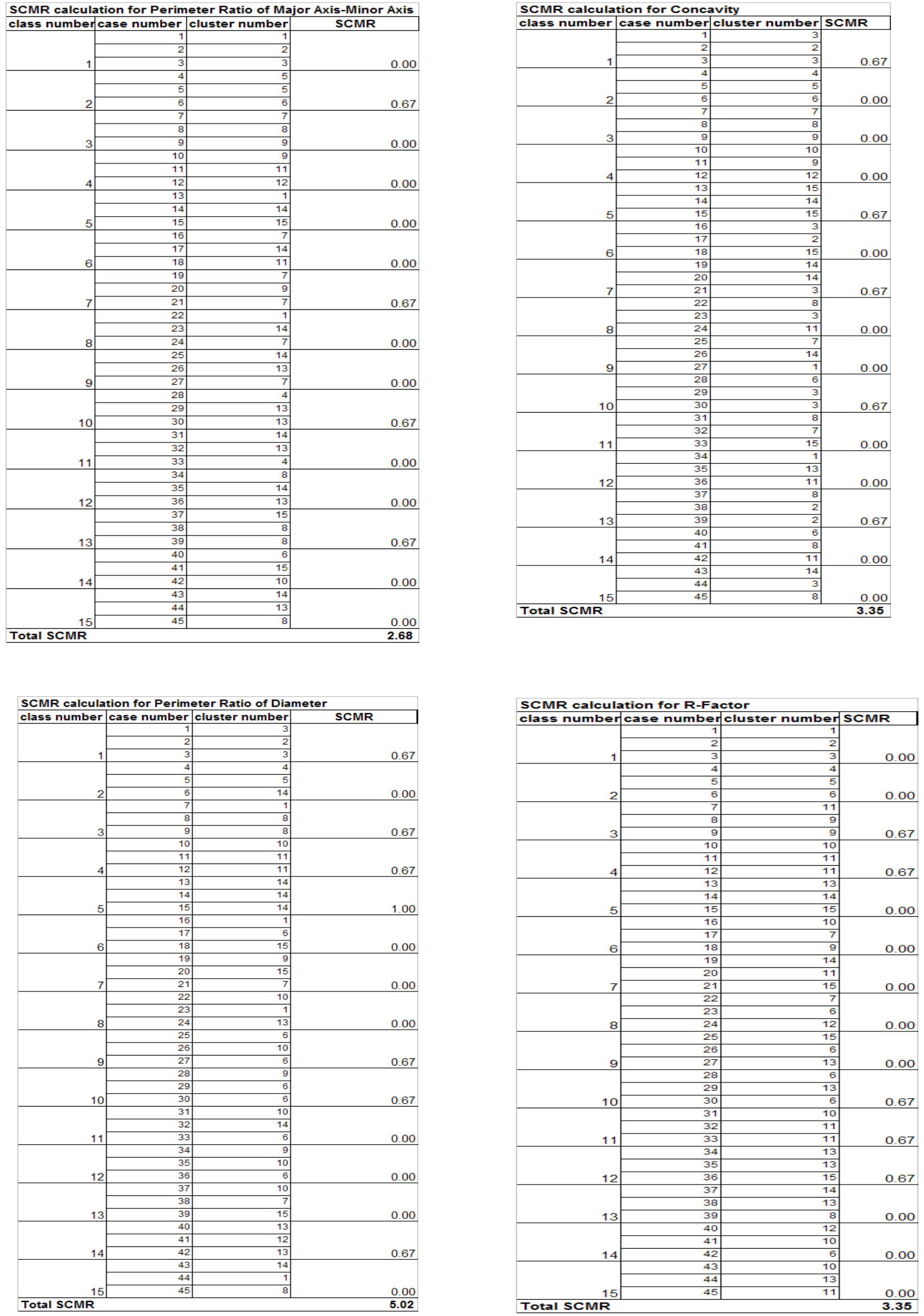
International Journal of Computer Applications Technology and Research Volume 2- Issue 5, 557 - 586, 2013, ISSN: 2319-8656

Table 8-SCMR calculation for individual fruit features

SCMR calculation for Branch Length

class number case number cluster number SCMR

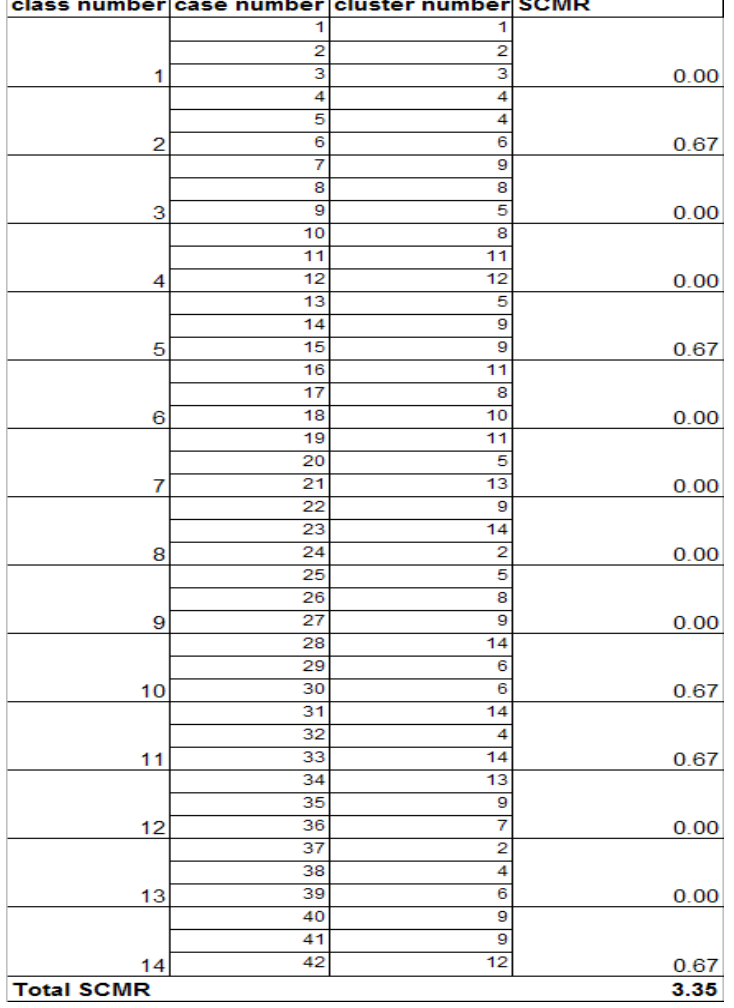

SCMR calculation for Branch Width

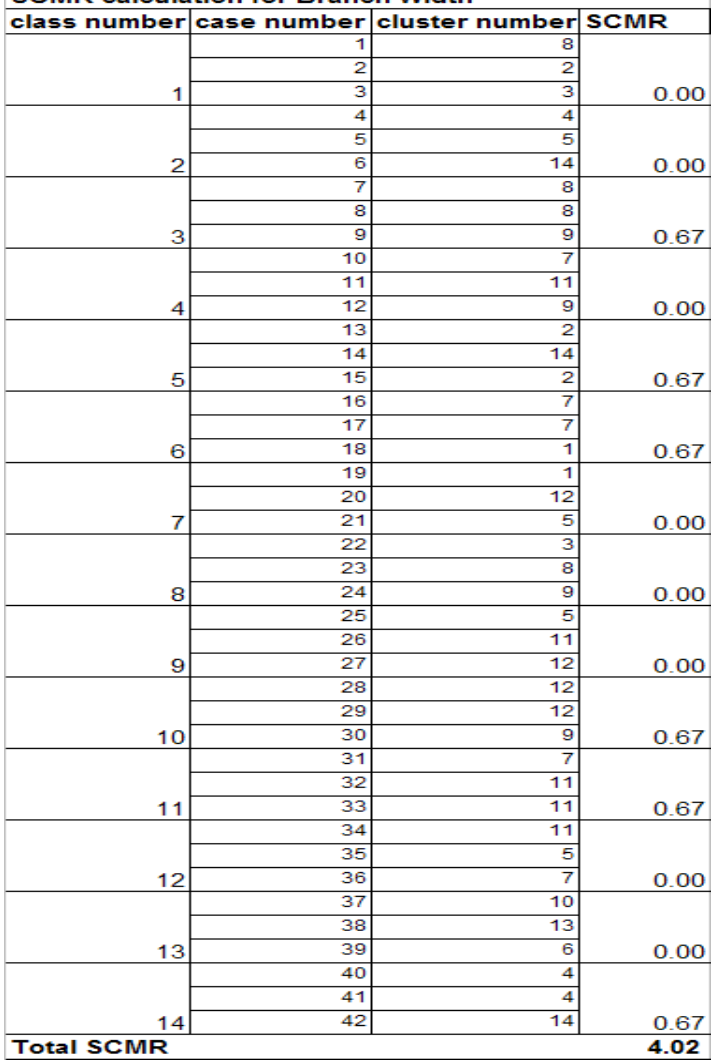

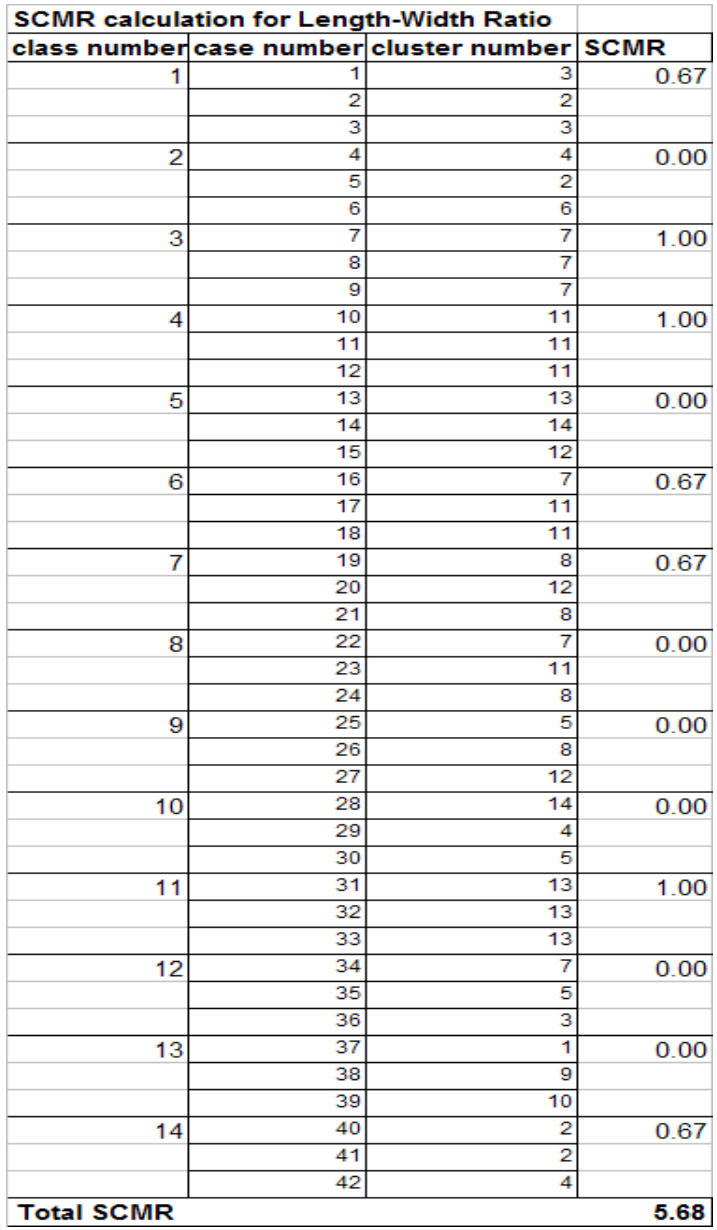

SCMR calculation for Area

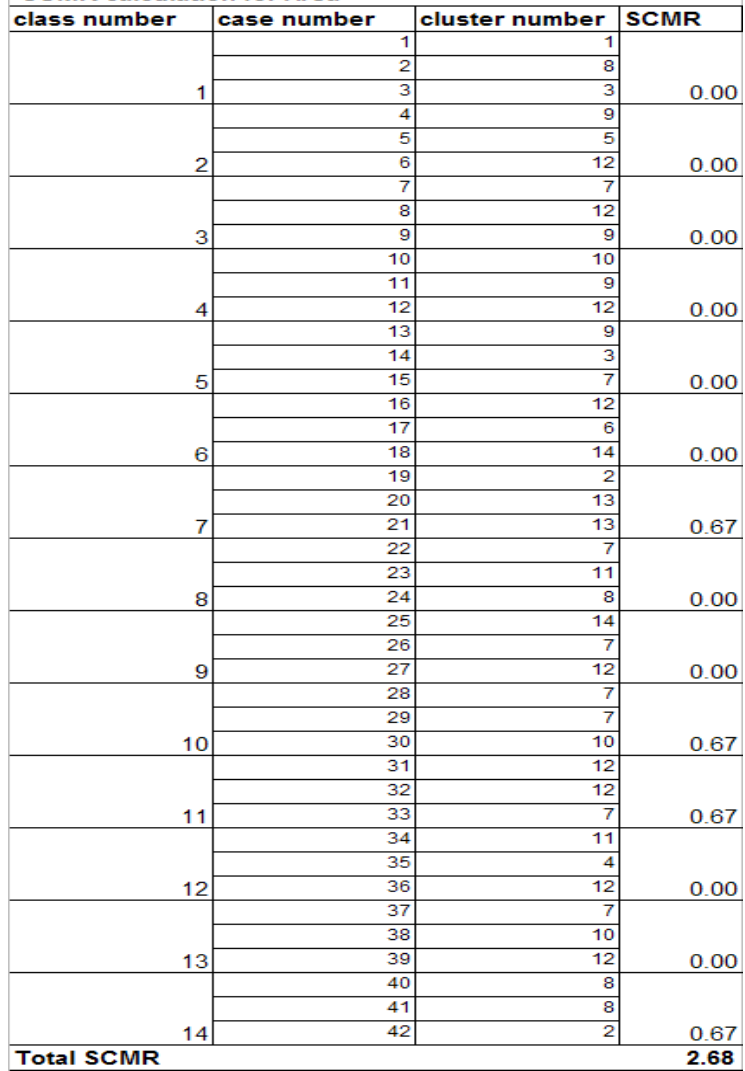


International Journal of Computer Applications Technology and Research Volume 2- Issue 5, 557 - 586, 2013, ISSN: 2319-8656
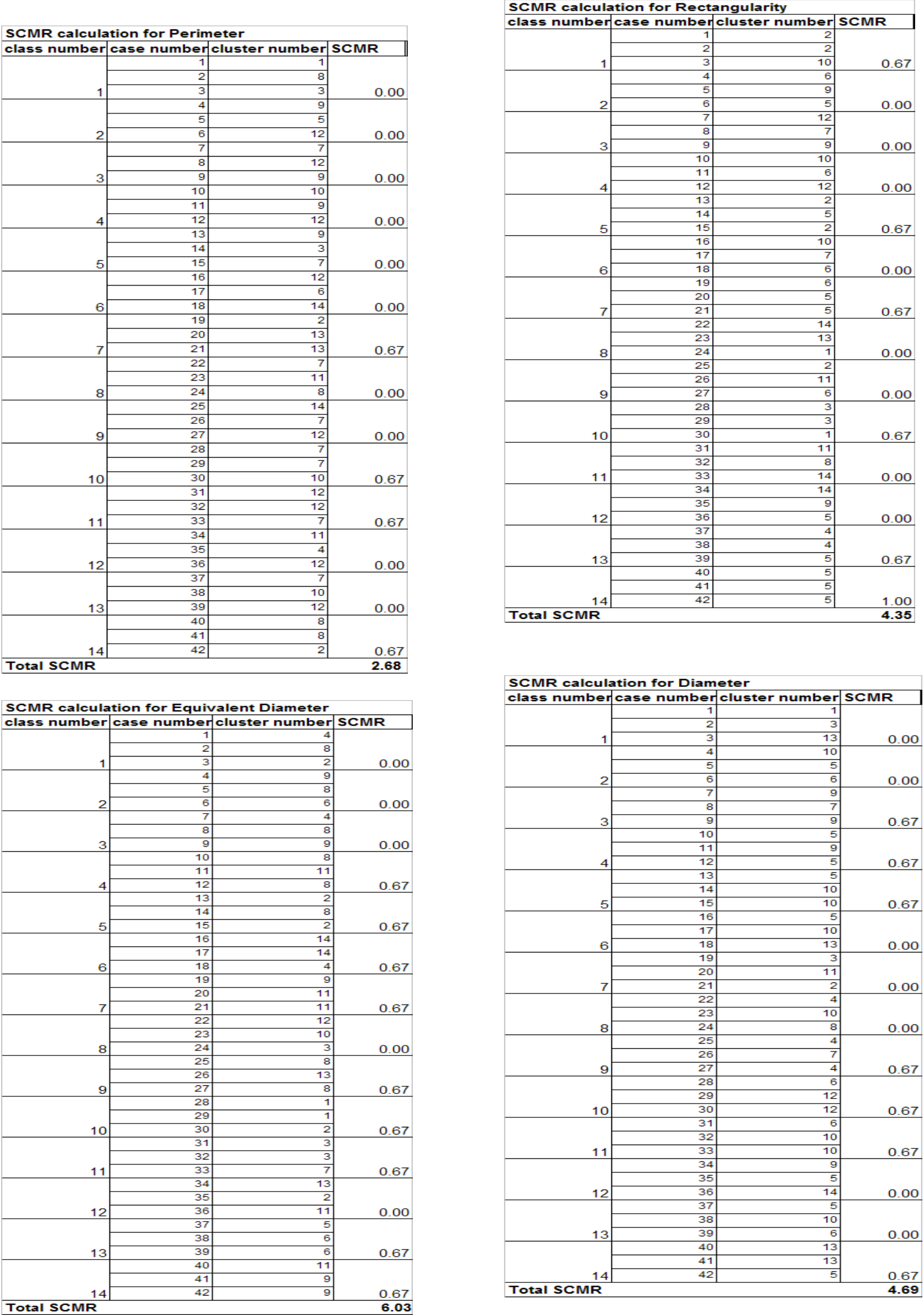
International Journal of Computer Applications Technology and Research Volume 2- Issue 5, 557 - 586, 2013, ISSN: 2319-8656

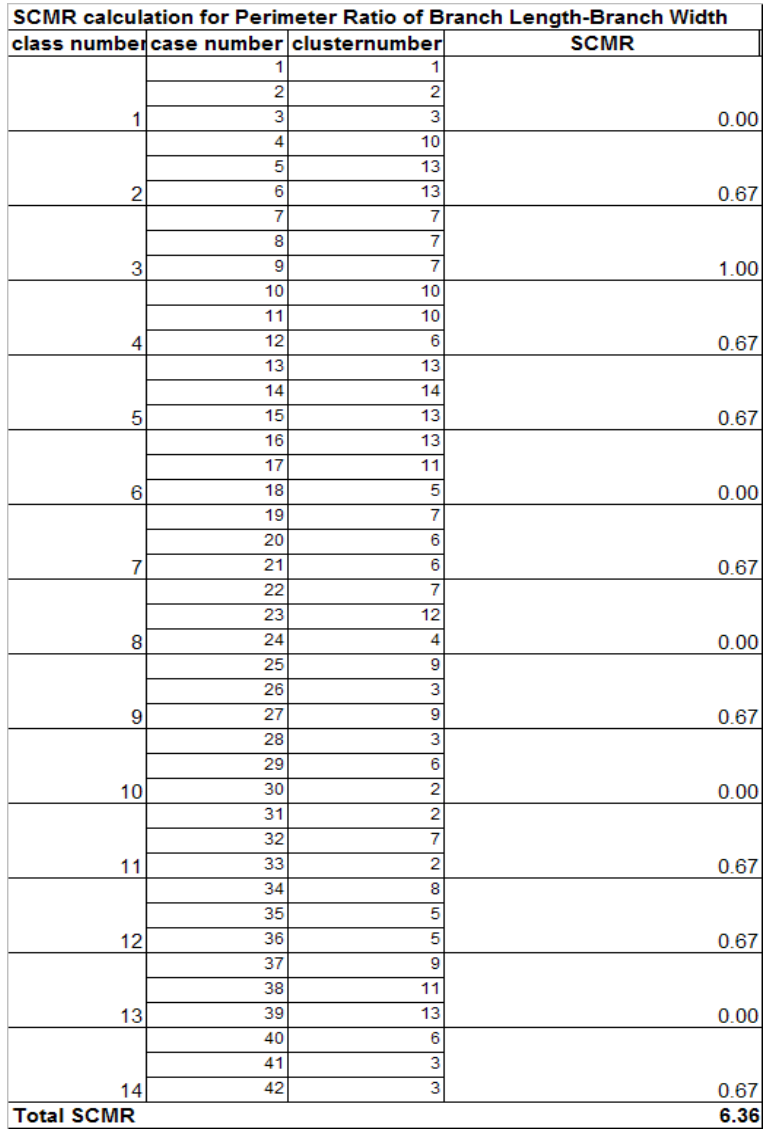

SCMR calculation for Perimeter Ratio of Diameter

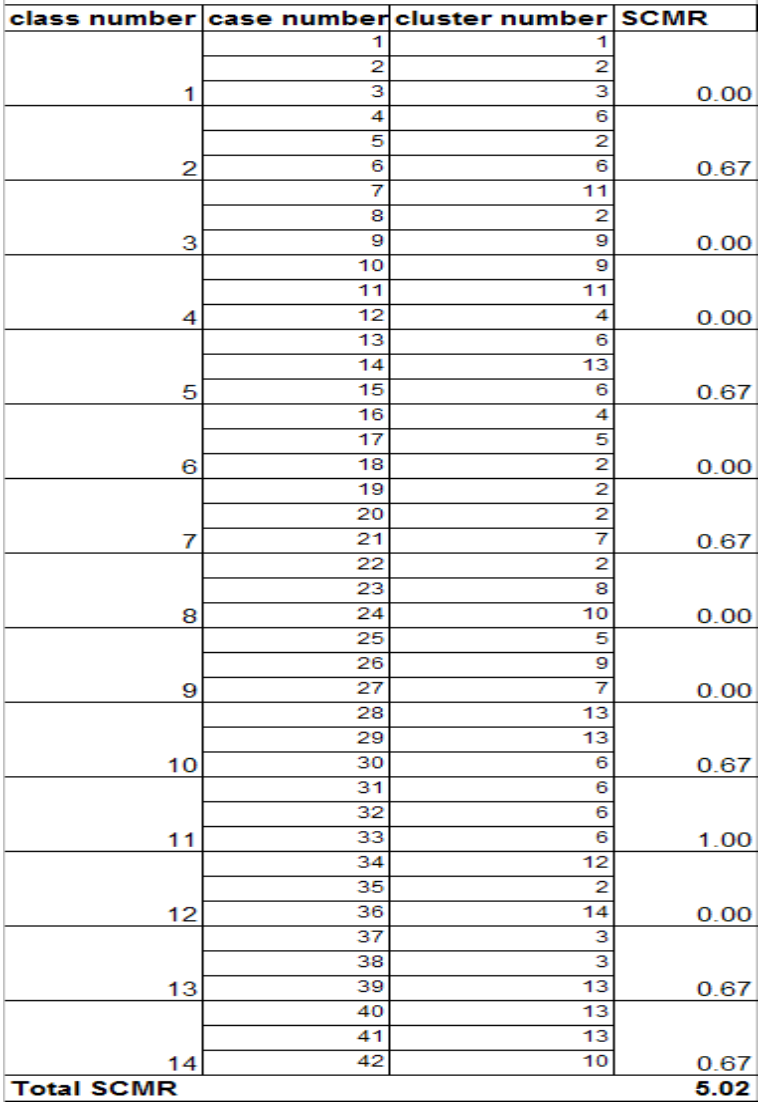

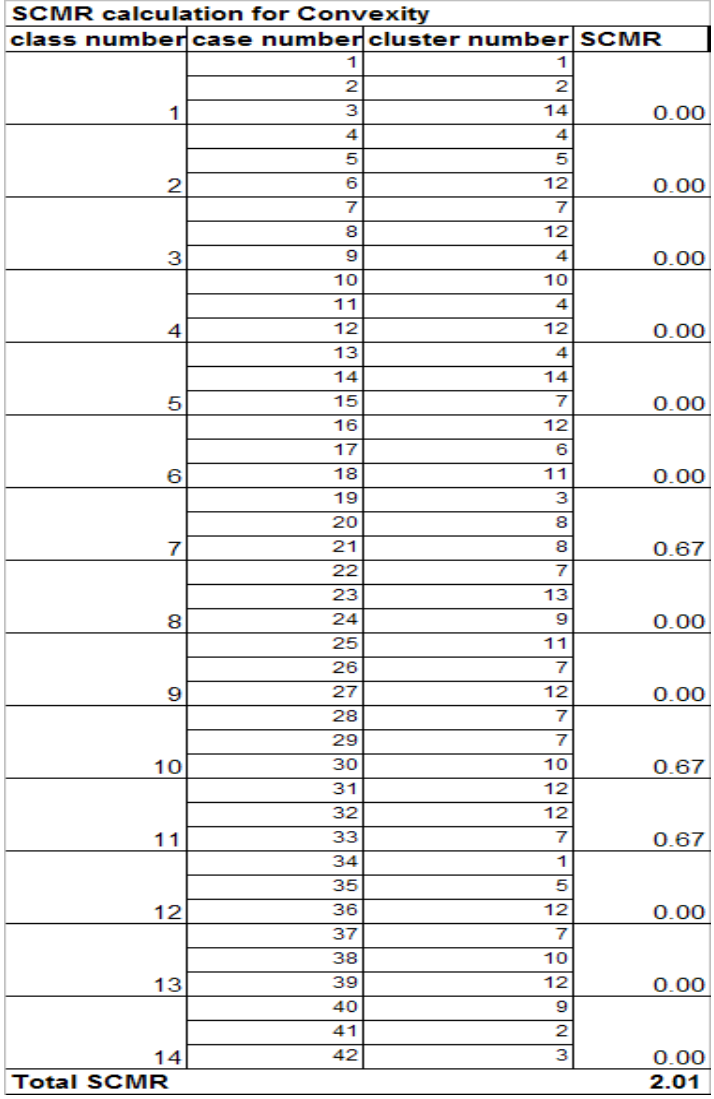

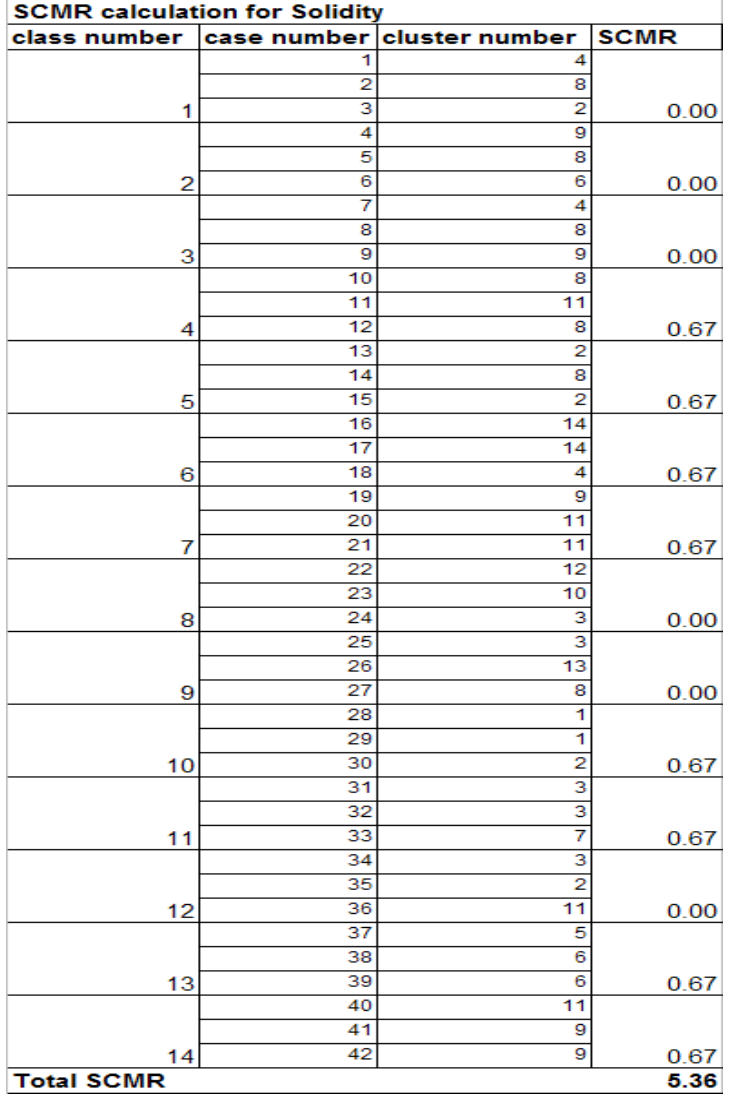


SCMR calculation for On Pixels

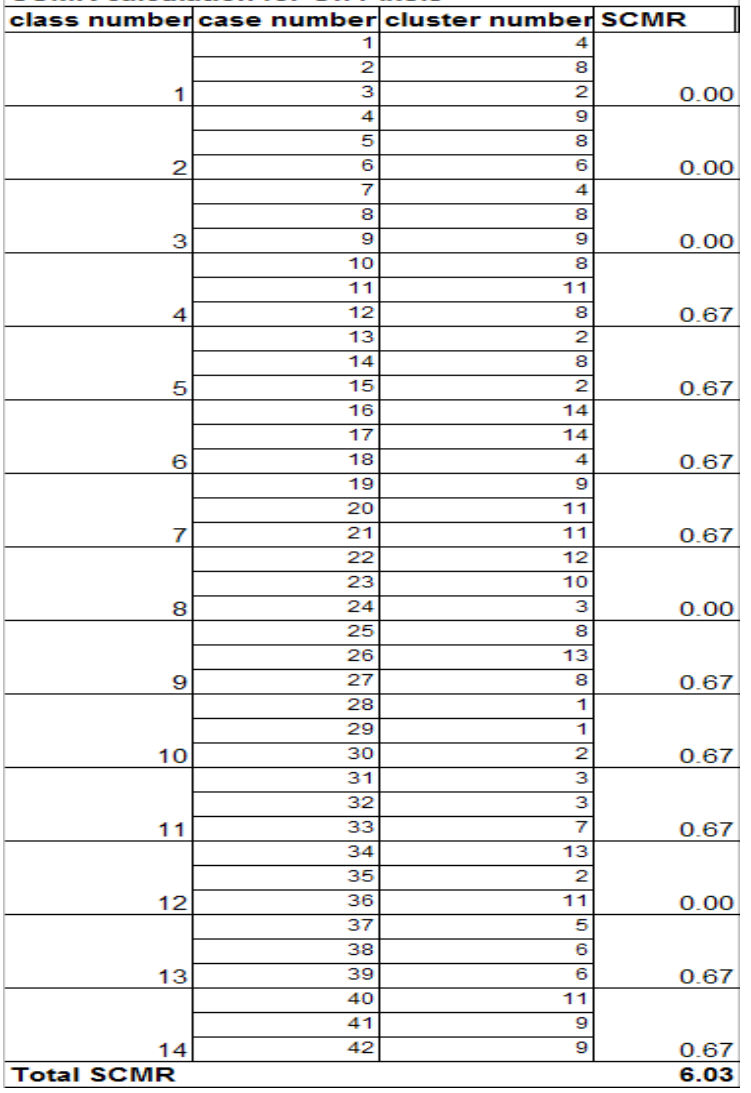

SCMR calculation for Narrow-Factor

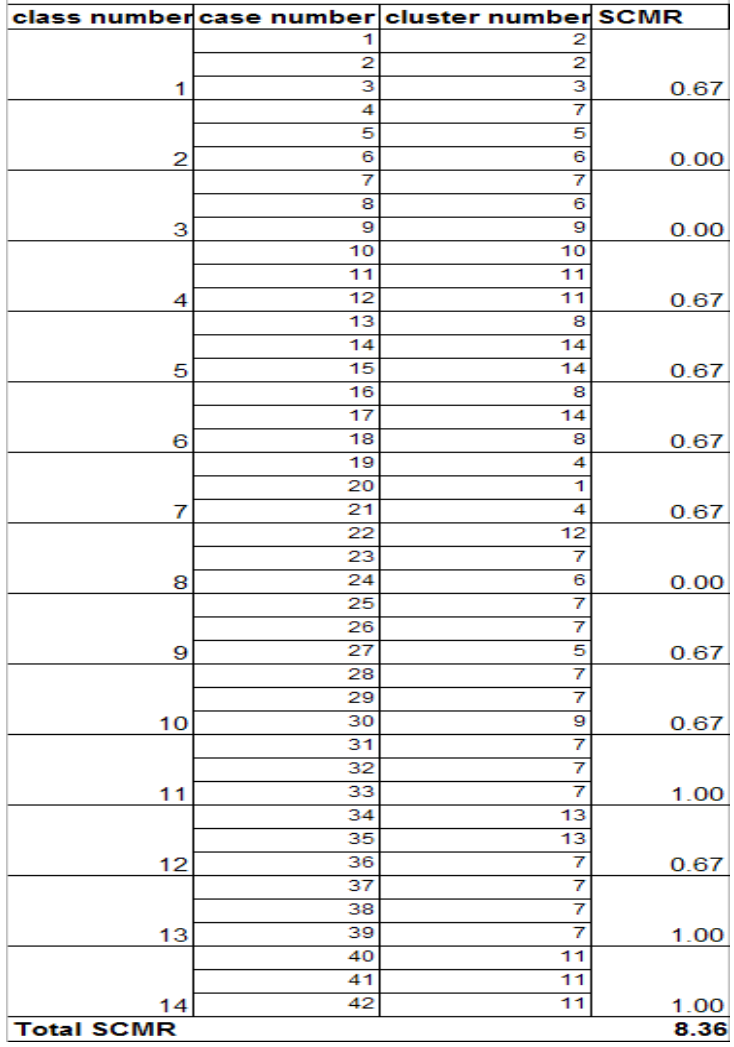

Total SCMR value of each of the leaf features (Table 9) and fruit features (Table 10) defines the fact that 'Minor Axis'(Total SCMR value 5.35)' and 'Major Axis'(Total SCMR value 0.67) are the leaf features with highest and lowest Total SCMR value. Hence 'Minor Axis' and 'Major Axis' are the most important and least important leaf features in terms of cluster formation respectively. Where as fruit feature having the highest and lowest importance are 'Narrow-Factor'(Total SCMR value 8.36, highest among all fruit features) and 'Convexity' (Total SCMR value 2.01, lowest among all fruit features) respectively.

Table 9- Leaf features with their Total SCMR values

\begin{tabular}{|c|c|}
\hline FEATURE NAME & $\begin{array}{c}\text { TOTAL } \\
\text { SCMR }\end{array}$ \\
\hline Major Axis & 0.67 \\
\hline Minor Axis & 5.35 \\
\hline Aspect Ratio & 3.35 \\
\hline Eccentricity & 2.68 \\
\hline Area & 3.35 \\
\hline Rectangularity & 3.35 \\
\hline Diameter & 3.35 \\
\hline Compactness & 2.68 \\
\hline Perim eter Ratio of Major Axis-Minor Axis & 2.68 \\
\hline Perimeter Ratio of Diameter & 5.02 \\
\hline Concavity & 3.35 \\
\hline R-Factor & 3.35 \\
\hline
\end{tabular}

Table 10- Fruit features with their Total SCMR values

\begin{tabular}{|c|c|}
\hline FEATURE NAME & $\begin{array}{c}\text { TOTAL } \\
\text { SCMR }\end{array}$ \\
\hline Branch Length & 3.35 \\
\hline Branch Width & 4.02 \\
\hline Length Width Ratio & 5.68 \\
\hline Area & 2.68 \\
\hline Perimeter & 2.68 \\
\hline Equivalent Diameter & 6.03 \\
\hline Rectangularity & 4.35 \\
\hline Diameter & 4.69 \\
\hline Perimeter Ratio of Branch Length-Branch Width & 6.36 \\
\hline Perimeter Ratio of Diameter & 5.02 \\
\hline Convexity & 2.01 \\
\hline Solidity & 5.36 \\
\hline On Pixels & 6.03 \\
\hline Narrow-Factor & 8.36 \\
\hline
\end{tabular}

\subsection{Two-step Clustering Results}

Two-step clustering algorithm has used 12 and 14 number of feature variables of tomato leaf and fruit as input and $15 \& 14$ final clusters of leaf and fruit are produced (Figure 1). 
Model Summary

\begin{tabular}{|l|l|}
\hline Algorithm & TwoStep \\
\hline Inputs & 12 \\
\hline Clusters & 15 \\
\hline
\end{tabular}

\section{Model Summary}

\begin{tabular}{|l|l|}
\hline Algorithm & TwoStep \\
\hline Inputs & 14 \\
\hline Clusters & 14 \\
\hline
\end{tabular}

Figure 1: Summary of Two-step clustering

Figure 2 shows the quality of the cluster formation of tomato leaf and fruit based on Silhouette measure of cohesion and separation. Silhouette measure is obtained with in the range of -1.0 to 1.0. Silhouette measure of greater than 0.5 signifies that the cluster formation is of a good quality. So from Figure 2, it is obvious that Two-step clustering has formed good quality clusters from sample patterns of tomato leaf and fruit.
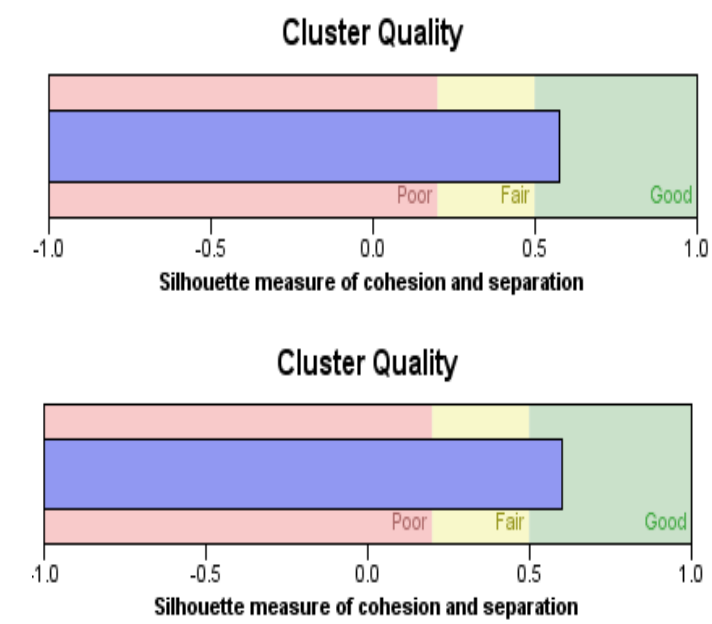

Figure 2: Cluster formation quality for leaf and fruit.

Number of patterns placed in a cluster defines the size of that cluster. Figure 3 depicts the pie chart representation of the leaf and fruit clusters according to their sizes expressed in terms of percentage of the total number of leaf and patterns.
Cluster Sizes
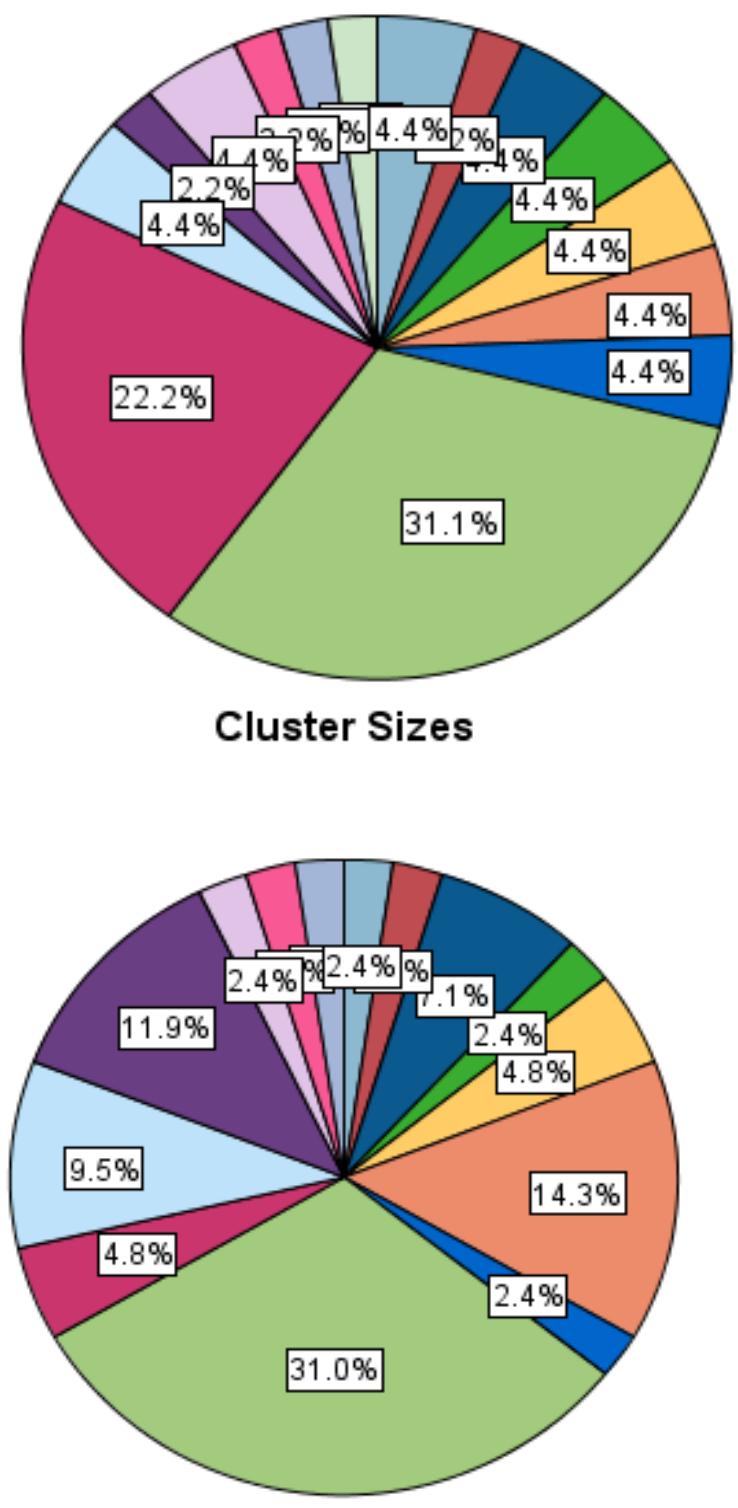

Figure 3: Pie chart of leaf and fruit clusters (size wise)

All features don't contribute in the same manner in the cluster formation process. Some features contribute highly where contribution of some features may be low. Features with higher level of contribution have higher importance in the formation of clusters. Figure 4 shows the top-down arrangement of leaf and fruit features in descending order of their importance in cluster formation. According to Figure 4, Compactness and Major Axis are the most important and least important leaf features. Where as Rectangularity and Branch Length are the fruit features with highest and lowest importance. 
Predictor Importance

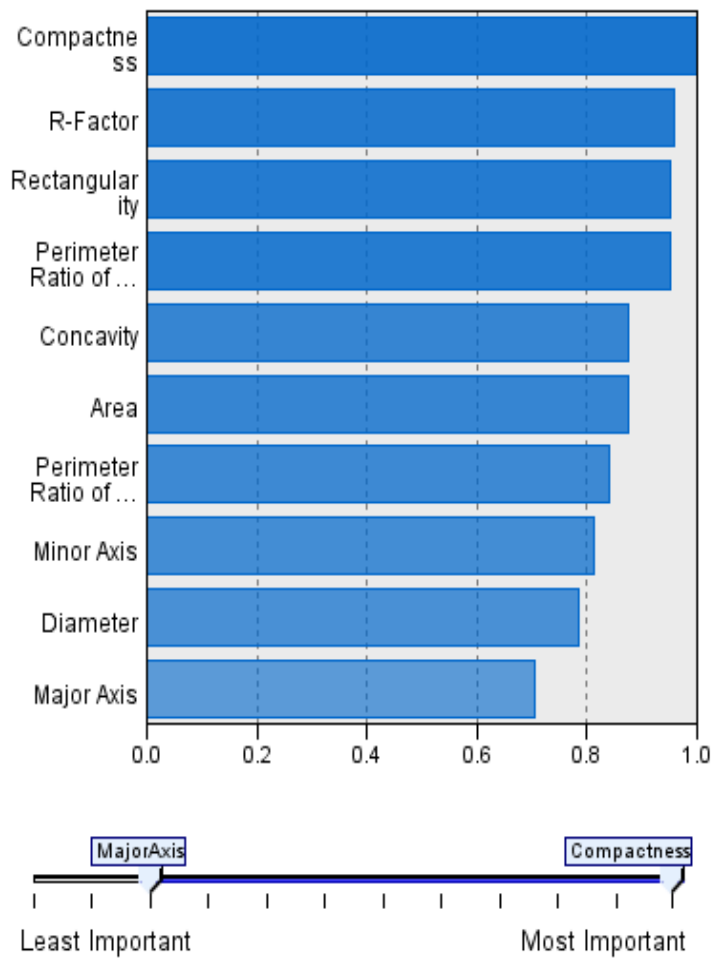

\section{CONCLUSION}

The scheme for K-Means and Two-step clustering algorithm to discriminate the tomato leaf, fruiting habit image samples along with morphological feature importance has been introduced. Formation of valid clusters assures the successful execution of the clustering techniques on the feature set and there by reflecting the categorical distribution of tomato species. Feature importance calculation through SCMR measure declares Minor Axis and Narrow-Factor as the respective leaf and fruit features upon which best cluster formation is observed. Where as according to Two-step clustering, Compactness and Rectangularity are the most important leaf and fruit feature as per as cluster formation capability is concerned. Considering this phenomena, one of the aspects of future work is to use these features to validate a large volume image dataset of tomato leaves and fruits. Another important futuristic aspect is to build up a leaf / fruit categorization system with relevant feedback mechanisms to help the persons related to cultivation process and collection of feedback from them for the enhancement of the system. Also applying the other renowned clustering methods like fuzzy clustering, neural network based clustering on the sample data set and hence analyzing the result is a future work.
Predictor Importance

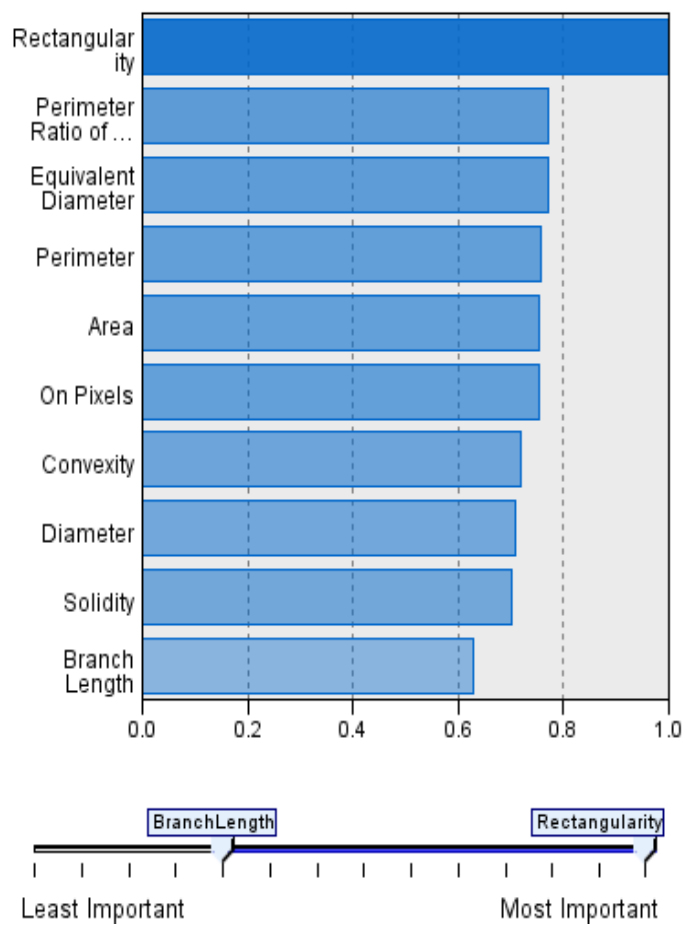

Figure 4: Order of Importance of leaf and fruit features

\section{REFERENCES}

[1] A.Hazra, K.Deb, S.Kundu, P.Hazra,"Shape Oriented Feature Selection for Tomato Plant Identification", International Journal of Computer Applications Technology and Research,Volume 2-Issue 4, 2013, pages 449-454.

[2] S.Kundu, A.Hazra, K.Deb, P.Hazra, "Dimensionality Reduction of Morphological features of Tomato Leaves and Fruiting Habits", IEEE International Conference on Communications, Devices and Intelligent Systems(CODIS 2012),pages 608-611

[3] Gregory A. Wilkin, Xiuzhen Huang,'K-Means Clustering Algorithms: Implementation and Comparison", IEEE Second International Multisymposium on Computer and Computational Sciences, 2007, pages 133-136.

[4] Li-Qing Li, Qiao Liu, Han-qing Zhou,"Research on Patient Satisfaction Degree Evaluation of Three A-level Hospital in Jiangxi Province Based on Cluster Analysis", IEEE International Conference on Information Management, Innovation Management and Industrial Engineering, 2011, pages 563-567.

[5] Jie Yao, "Research on the Application of K-means cluster analysis in undergraduate instructional management", IEEE International Conference on Advanced Computer Control, 2008, pages 628-631. 
International Journal of Computer Applications Technology and Research Volume 2- Issue 5, 557 - 586, 2013, ISSN: 2319-8656

[6] Bin Lie, Huichao Zhang, Huiyu Chen, Lili Liu, Dingwei Wang, "A K-means Clustering Based Algorithm for Shill Bidding Recognition in Online Auction", IEEE 24th Chinese Control and Decision Conference(CCDC 2012), pages 939-943.

[7] Shuhua Ren, Alin Fan, " $K$-means Clustering Algorithm Based On Coefficient of Variation", IEEE 4th International Congress on Image and Signal Processing, 2011, pages 2076-2079.

[8] M.Narasimha Murty, V.Susheela Devi, "Pattern Recognition An Algorithmic Approach Undergraduate Topics in Computer Science",Springer 2011, Universities Press (India) Pvt.Ltd. 207-229

[9] Andrew R. Webb, "Statistical Pattern Recognition", Second Edition, 2002, John Wiley \& Sons,Ltd, 361-402 